\title{
Linking Particle Dynamics to Intracellular Micromechanics in Living Cells
}


The research described within this thesis was carried in the Physics of Complex Fluids group at the MESA+ Institute for Nanotechnology at the University of Twente, Enschede, the Netherlands. The project is financially supported by SRO cell stress of MESA+ institute.

Committee members:

\section{Chairman}

Prof. dr. G. van der Steenhoven

\section{Promotor}

Prof. dr. F. Mugele

\section{Assistant promoter}

Dr. M.H.G. Duits

\section{Members}

Prof.dr. A. Bausch

Prof.dr. W.J. Briels

Prof.dr. V. Subramaniam

Prof.dr. I. Vermes

Dr. S.A.Vanapalli

Title: Linking Particle Dynamics to Intracellular Micromechanics in Living Cells

Author: Yixuan Li

ISBN: 978-90-365-2893-1

Publisher: Woehrmann Printing Service, Zutphen, the Netherlands

Cover picture: a Hmec-1 cell injected with fluorescent tracer particles.

Copyright ( 2009 by Yixuan Li, Enschede, the Netherlands 


\title{
LINKING PARTICLE DYNAMICS TO INTRACELLULAR MICROMECHANICS IN LIVING CELLS
}

\author{
DISSERTATION
}

to obtain

the degree of doctor at the University of Twente, on the authority of the rector magnificus, prof.dr. H. Brinksma, on account of the decision of the graduation committee, to be publicly defended on Wednesday the $11^{\text {th }}$ of November 2009 at 15.00

\author{
by \\ Yixuan Li
}

born on the $29^{\text {th }}$ of June 1973

in Jilin, China 
Dit proefschrift is goedgekeurd Door de promotor

Prof. Dr. F. Mugele

en de assistent-promotor

Dr. Michel, H.G. Duits 


\section{Table of Contents}

1 Introduction............................................................. 1

1.1 Cell mechanics...................................................................... 2

1.2 Molecular origins.................................................................. 2

1.3 Theoretical models................................................................... 5

1.3.1 Rheology ..........................................................

1.3.2 Viscoelastic behavior of complex fluids ..................... 5

1.3.3 Mechanical models for the cell........................................ 7

1.3.4 Active forces.......................................................

1.3.5 Summarizing statements on theoretical modeling................10

1.4 Experimental techniques in cell mechanics ...................................10

1.4.1 Overview.........................................................10

1.4.2 Particle tracking.......................................12

1.4.3 Atomic force microscopy............................................. 17

1.5 This thesis .............................................................................................21

References.................................................................................23

2 Materials and Methods...................................................30

2.1 Cells............................................................................. 31

2.1.1 Cell culture...........................................................31

2.1.2 Immunofluorescent staining...............................32

2.1.3 Drug and temperature interventions ..................................33

2.1.4 Temperature control and calibration for IPT \&AFM exp...34

2.2 Ballistic microinjection ..................................................................34

2.2.1 Preparation of nanoparticles and macrocarriers.....................35

2.2.2 Ballistic injection of nanoparticles ........................36

2.3 Intracellular particle tracking (IPT)............................................37

2.3.1 Phase Contrast \& Confocal Scanning Laser Microscopy

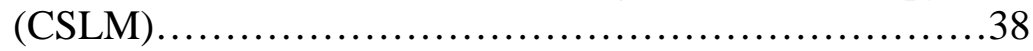

2.3.2 Probe types.................................................

2.3.3 Multiple particle tracking.............................................39

2.4 Combined AFM-CSLM experiments................................................40

2.4.1 Force distance experiments...............................42

References.............................................................43

\section{Mapping of Spatiotemporal Heterogeneous Particle Dynamics} in Living Cells..................................................45

3.1 Introduction................................................................... 46 


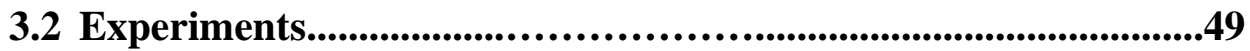

3.2.1 Cell culture...............................................49

3.2.2 Probe types .......................................49

3.2.3 Intracellular particle tracking............................50

3.2.4 Computer simulations.................................50

3.3 Particle tracking.................................................................50

3.3.1 Mean squared displacement functions..........................50

3.3.2 Segmentation.........................................52

3.3.3 Spatial and time dependence...........................53

3.3.4 Time auto correlation function...........................54

3.3.5 Spatial cross correlation function...........................55

3.3.6 Time variance............................................56

3.3.7 Spatial variance..........................................57

3.4 Results..............................................................57

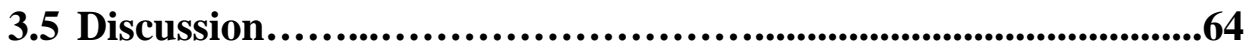

3.5.1 Implications for particle tracking studies......................64

3.5.2 Application of individual trajectory analysis..............65

3.5.3 Comparison with other particle tracking methods...........67

3.6 Conclusions and outlook......................................................68

References....................................................................69

\section{On the Origins of the Universal Dynamics of Endogenous}

Granules in Mammalian Cells......................................72

4.1 Introduction............................................................... 73

4.2 Materials and Methods...........................................................73

4.2.1 Cell Culture and intracellular probes............................73

4.2.2 Interventions........................................74

4.2.3 Multiple particle tracking experiments....................74

4.3 Results and discussions.......................................................75

4.3.1 Universal diffusive dynamics of endogenous granules are driven by cytoskeletal active forces ...........................75

4.3.2 Probing the origins of the non-equilibrium dynamics of endogenous granule....................................78

4.3.3 Microtubule polymerization dynamics drives the motion of endogenous granules in mammalian cells..................80

4.4 Conclusions. .84

References..........................................................................85

\section{Dynamics of Ballistically Injected Latex Particles in Living} Human Endothelial Cells. 


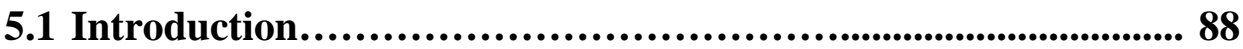

5.2 Materials and methods.................................................................90

5.2.1 Cell culture...................................................99

5.2.2 Probe types...........................................99

5.2.3 Ballistic injection......................................99

5.2.4 Drug interventions on cytoskeleton and myosin motors....91

5.2.5 Immunofluorescence microscopy ...........................91

5.2.6 Multiple particle tracking....................................91

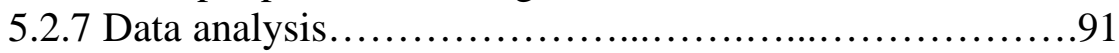

5.3 Results.................................................................................93

5.3.1 Ensemble dynamics of BIP...................................93

5.3.2 Interpretative approach...................................94

5.3.3 Drug interventions aimed at the actin network...............95

5.3.4 Drug interventions aimed at microtubules..................97

5.3.5 Individual dynamics of BIP..............................98

5.3.6 Drug interventions aimed at disabling active processes...101

5.4 Discussion

5.4.1 Micro-environment of the BIP in the living cytoskeleton.102

5.4.2 Driving forces for BIP.................................102

5.5 Conclusions and outlook............................................................102

References............................................................................104

\section{Intracellular Particle Tracking as a Tool for Tumor Cell}

Characterization...............................................107

6.1 Introduction...................................................................108

6.2 Materials and methods............................................................109

6.2.1 Cell culture....................................................109

6.2.2 Nanomechanical measurements with AFM.................110

6.2.3 Intracellular Probes.......................................110

6.2.4 Particle tracking using optical microscopy .................111

6.3 Results and discussion.................................................................112

6.3.1 Intracellular Particle Tracking....................................112

6.3.2 Atomic Force Microscopy................................115

6.4 Further considerations...............................................................117

6.4.1 Comparison of IPT and AFM...............................117

6.4.2 Implementation perspectives for IPT...............................118

6.5 Summary.............................................................................119

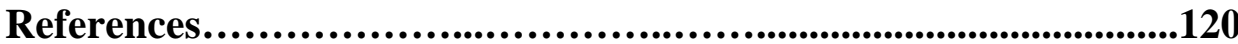


7 Conclusions and outlook......................................123

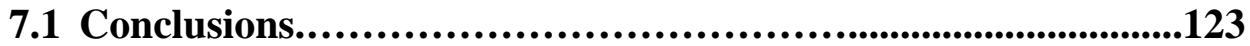

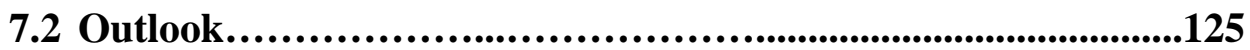

7.2.1 IPT for fundamental cell-mechanical studies................125

7.2.2 IPT for potential applications in nanomedicine..............127

7.2.3 Miniaturization and automatization .......................127 


\section{List of Abbreviations}

1PMR One-point particle tracking microrheology

2PMR Two-point particle tracking microrheology

ABPs Actin-binding proteins

AF Actin Filament

AFM Atomic force microscopy

AMC Acto-myosin contractility

AN Actin network

ATP Adenosine triphosphatase

BIPs Ballistically injected particles

BSA Bovine serum albumin

CSLM Confocal scanning laser microscopy

DAPI 4',6-diamidino-2-phenylindole, a fluorescent dye for nuclear staining

DiI Vybrant cell lipid staining solution

DMEM Dulbecco's modified eagel medium

E*

Apparent Young's modulus

EGM Endothelial cell growth medium

EGs Endogenous granules

FBS Fetal bovine serum

FDT Fluctuation-dissipation theorem

FITC Fluorescein isothiocyanate

GG Gelatin gel

GSER Generalized Stokes-Einstein relation

HMEC-1 human microvascular endothelial cell line

IDL Interactive Data Language

IF Intermediate filament

iMSD Individual Mean Squared Displacment

IPT Intracellular particle tracking

LA Latrunculin A

MAPs Microtubule associated proteins

MCF-10A Benign human breast fibrocystic epithelial cell line

MCF-7 Malignant human breast epithelial adenocarcinoma cell line

ME Micro-Environment

MPTM Multiple particle tracking microrheology

MSD Mean squared displacement 
MT Microtubule

Nile Red lipophilic dye for EGs staining

PA-S Benign human pancreas adenocarcinoma cell line Patu8988S

PA-T Malignant human pancreas adenocarcinoma cell line Patu8988T

PBS Phosphate buffer saline

PTM Particle tracking microrheology

SGM Soft-glassy material

SGR Soft-glassy rheology

SL Simple liquid 


\section{Chapter 1}

\section{Introduction}

This chapter introduces the field of cell mechanics and the aim of the thesis project. An overview is given about experimental approaches in cell mechanical studies. Specifically the applications of intracellular particle tracking and Atomic Force Microscopy in the studies of cell mechanics are discussed in detail. 


\subsection{Cell mechanics}

Cell mechanics $(1,2)$ is the field of studying how cells detect, modify and respond to mechanical cues from their environment. Cells are a very fascinating demonstration of nature's intricate and well-coordinated micro-mechanical objects. Both internal and external mechanical responses can occur in living cells. It is known that cells exert internally generated forces on the materials to which they adhere through a transmembrane protein called integrin (3). In turn, the stiffness of the substrate onto which cells are attached, also affects the cellular structure, motility and growth. Besides this behavior, cells can also detect the imposition of forces arising from the external tissue or fluid environment, through mechanically sensitive molecules on the cell surface such as receptor proteins or ion channels. This behavior is termed mechanosensing (4). As a consequence, these external mechanical stimuli can activate a series of biochemical responses, called mechanotransduction $(5,6)$. These responses often also lead to a mechanical response, originating from a deformation or reorganization of the cytoskeletal network.

It has turned out that such integrative mechanical behavior is crucial to maintain the proper morphology and functioning of cells. A good example is given by endothelial cells, for which the interplay of cell connectivity, the internal cytoskeletal networks, nuclei and the external cell matrix, generates a superb ability to withstand shear stress and blood pressure. Conversely, a dysfunction in any of these elements, becomes manifest in mechanical dysfunction leading to cardiovascular diseases, for example atherosclerosis. Another example is that of malignant cancer cells, which turn out to be much softer and therefore more deformable than healthy cells. This property is believed to have intrinsic connection with their metastatic nature. These are just examples; also more generally it is becoming increasingly clear that cell mechanics plays a central role in embryogenesis, tissue physiology, as well as the pathogenesis of a wide variety of diseases (7-9).

\subsection{Molecular origins}

To understand the origins of a cell's mechanical properties, the starting point is to study the intracellular microstructure, also known as the cytoskeleton. This 'backbone' of the cell is a protein fibre network which is composed of three chemically and mechanically very different biopolymers $(7,10)$ : actin filaments, microtubules and intermediate filaments: 


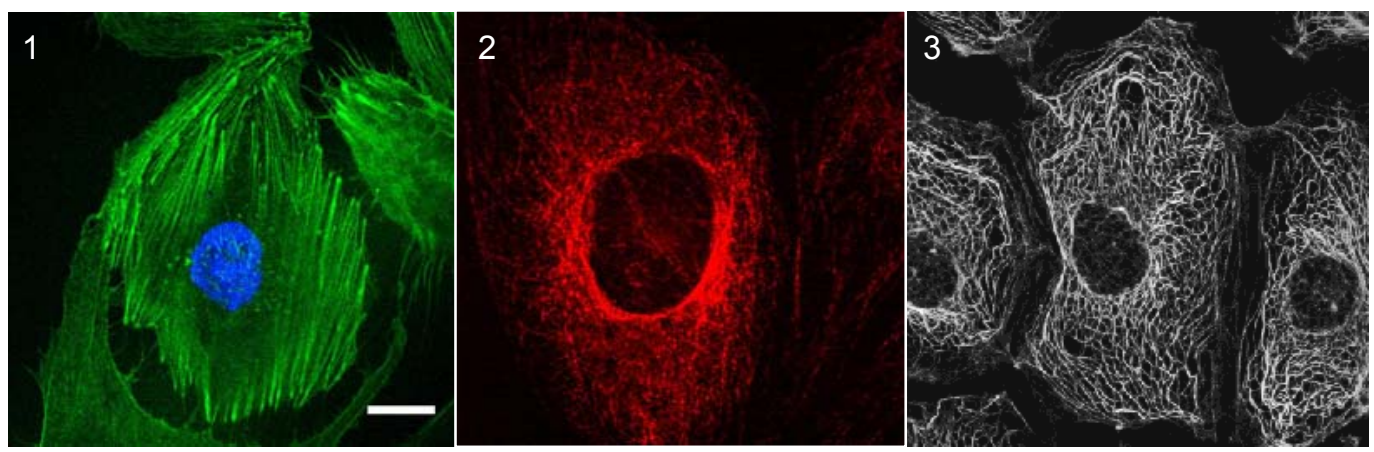

Fig.1.1, Fluorescent staining images of 3 major cytoskeletal components.1, actin network in human endothelial cells stained with FITC-pholloidin, and nucleus in blue (DAPI); 2, microtubule network in human endothelial cells in red;3, Intermediate filament network of keratin in epithelial cells (courtesy of W. W. Franke). Scale bar is $10 \mu \mathrm{m}$ for all images.

Actin filaments (AFs):

Actin filaments (F-actin or microfilaments) are two-stranded helical polymers with a diameter $6 \sim 8 \mathrm{~nm}$ composed of the actin monomers. They are spread over the entire cell, but mostly concentrated in the cortex region beneath the cell membrane. The free monomers of actin are globular molecules and therefore called G-actin. F-actin and G-actin are a prominent part of the intracellular proteins in cells, occupying typically $\sim 1-10 \mathrm{wt} \%$ of the total protein content in non-muscle cells. AFs play an important role in cell motility, integrity and adhesion. In in vitro studies, AFs were found to have a persistence length $L_{\mathrm{p}}$ of $10-15 \mu \mathrm{m}$ and a bending stiffness $K_{\mathrm{B}}$ of $7 \times 10^{-26} \mathrm{Nm}^{2}$.

\section{Microtubules(MTs):}

Microtubules are relatively long, hollow cylinders composed of tubulin heterodimers as the basic subunit. The inner diameter of MTs is $14 \mathrm{~nm}$, and outer diameter $25 \mathrm{~nm}$. MTs function in cell migration, especially during mitosis, and contribute to cell integrity. The persistence length of MTs is $\sim 6 \mathrm{~mm}$ in vitro, however, in vivo this number can be several orders of magnitude smaller. MTs are much more rigid than AFs with a bending stiffness $K_{\mathrm{B}}$ of $2.6 \times 10^{-23} \mathrm{~N} / \mathrm{m}^{2}$.

Intermediate filaments (IFs):

Among the three major components of cytoskeleton, IFs have been the least studied. They are ropelike fibers with a diameter $\sim 10 \mathrm{~nm}$. IFs constitute roughly $1 \%$ of total proteins in most cells, but this can rise up to $85 \%$ in cells such as epidermal keratinocytes and neurons. The major functions of IFs are related to structural integrity, for example of the nuclear lamina which is just beneath the 
inner nuclear membrane. Other types of IFs, for example vimentin in endothelial cells is believed to provide mechanical rigidity to the cells. IFs have a persistence length of 1-3 $\mu \mathrm{m}$, with bending stiffness similar to Afs: $4-12 \times 10^{-27} \mathrm{~N} \mathrm{~m}^{2}$ in vitro.

In living cells, the cytoskeleton is continually remodeled through two major processes, which depend on biochemical energy conversion (i.e. require more than just thermal energy):

\section{Polymerization / depolymerization of biopolymers}

The assembly and disassembly of AFs occurs via a process called treadmilling in the presence of ATP. Hundreds of actin-binding proteins (ABPs) control linear elongation, shortening and architectural organization of actin filaments in response to signaling cascades set in motion by environmental cues. There are 3 major types of microfilaments: i) linear bundles, for example filapodia and contractile bundles; ii) two-dimensional networks, eg. lamellapodia; and iii) three-dimensional networks, such as the actin cortex.

Like AFs, also MTs undergo polymerization / depolymerization process in the presence of GTP. This is termed dynamic instability. The forces induced in this process can cause the buckling of MTs, however this buckling can also be diminished via lateral reinforcement of the surrounding medium (11). Microtubules can also be cross-linked by proteins, for example in axons, microtubules are bundled into a tight core of aligned filaments via microtubule associated proteins (MAPs).

IFs are generally believed to form a passive network that is not as dynamic as AFs and MTs since they are not acted on by ATP driven motors. In recent work (12) it was found that the IF network is predominantly elastic, albeit with a small but measurable viscous modulus.

\section{Force generation by molecular motor proteins}

Myosin motor families mediate ATP dependent relative sliding of actin filaments to generate forces that result in for example in muscle contraction, or contractile stresses in non-muscle cells, cell locomotion and control of cell shape. During this process, a transient complex called actomyosin complex is formed by the association of myosin motors with actin filaments. Two other types of motor proteins, kinesins and dyneins are microtubule associated motor proteins, which carry membrane enclosed organelles, such as mitochondria, Golgi stacks, or secretory vesicles and walk along the MT network to the appropriate locations in the cell. They are GTP dependent motor proteins and mainly responsible for 
intracellular vesicle transportation and organization of cellular substructure. The functionality of motor proteins is controlled via regulatory proteins or small ions. For instance, $\mathrm{Ca}^{2+}$ can interfere with the phosphorylation of myosin motors to enhance the contractility of the actomyosin complex.

The study of cytoskeleton mechanics is complicated due to the interplay between the mechanical properties of the passive biopolymer network and the active forces that are generated from the motor proteins, and polymerization / depolymerization of the biopolymers.

\subsection{Theoretical models}

The rich and complex mechanical behavior of cells has inspired researchers to develop several distinct models of the cytoskeleton. Most of these models have been restricted to predictions or descriptions of passive material properties, as are obtained in rheological experiments. To clarify the context of these models, we first review some basic rheological concepts.

\subsubsection{Rheology}

Rheology (13-16) is the field of studying how materials store and dissipate mechanical energy as a function of deformation and time scale. The most basic examples of rheological behavior are given by Newtonian liquids and Hookean solids. For a Newtonian liquid, the stress needed to reach a certain deformation rate is described by a proportionality constant known as the viscosity. A direct observation of our daily life, is that honey flows much slower (under gravity) than water; this is due to the higher viscosity. Analogously, for elastic solids the stress needed to achieve an equilibrium deformation is also described by a proportionality constant, known as the elastic modulus. Also the type of deformation should be specified in a rheological experiment; in most cases this is a shear deformation. Such deformations are applicable e.g. when materials flow through pipes or when particles move through the material.

In fundamental and applied rheology studies, the Newtonian liquid and Hookean solid are mainly used as reference cases. Most materials studied in rheology are so-called complex fluids, characterized by a structure at supra-molecular length scales (like polymer or particle networks). These structures give rise to a complex rheological behavior, which integrates elements of both liquid-like and solid-like behavior. A good example is linear viscoelastic behavior described by the frequency dependent shear modulus $\mathrm{G}^{*}(\omega)$. 


\subsubsection{Viscoelastic behavior of complex fluids}

Viscoelastic materials can show both liquid and solid like behaviors, depending on the timescale of the mechanical experiment. This ability applies to living cells, but also to particle suspensions, polymer solutions or polymer networks (15). Many of such complex materials have some kind of supramolecular structure, which can be deformed by external stress, and can recover. A good example is a polymer solution in which cross-linking can be achieved in a controllable way. In the absence of crosslinks, liquid like behavior is obtained, whereas in the presence of permanent links, an elastic gel is found. In the inter-mediate case of weak crosslinks, bonds between polymer chains are continually being formed and broken, giving rise to a transient network that has a relaxation time. If the external deformation is very fast then it will behave like an elastic solid, since there was not enough time to break and reform the bonds. But for slow deformations, the bonds will be broken (and new ones formed) before they could store significant deformation. Then the same material will behave like a fluid. Hence, the comparison of the experimental time scale and the relaxation time of the material determine which behavior is manifested.

A common method to describe such combined fluid/solid (viscoelastic) behavior is phenomenological models in which viscous behavior is represented by dashpots and elastic behavior by springs. The most commonly used models are the Maxwell and Kelvin-Voigt models, and their serial combination, known as the Burger model.

(A)

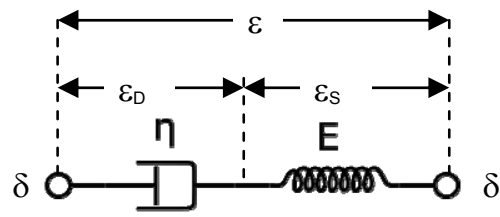

(B)

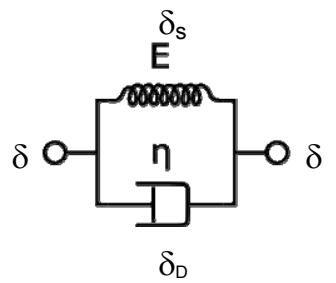

(C)

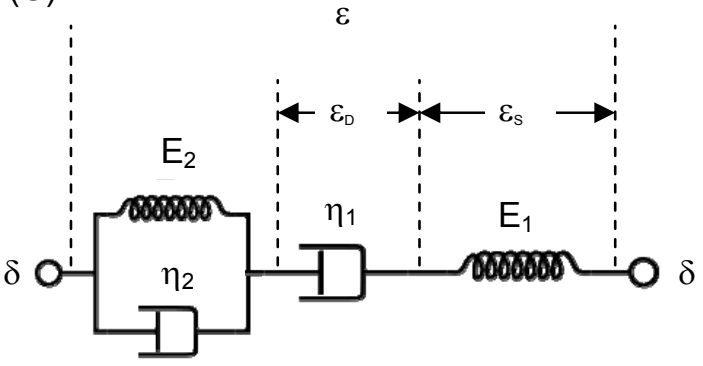

Fig. 1.2, Schematic view of viscoelastic models. (A) Maxwell model; (B) KlevinVoigt model; (C) Burger model.

We now briefly discuss these models. 
1, the Maxwell model can be represented by a purely viscous damper (dashpot, D) and a purely elastic spring (spring, S) connected in series, as shown in the Fig.1.2 (A). The model can be represented by the following equation:

$$
\begin{gathered}
\sigma_{\text {Total }}=\sigma_{D}=\sigma_{S} \\
\varepsilon_{\text {Total }}=\varepsilon_{D}+\varepsilon_{S} \\
\frac{d \varepsilon_{\text {Total }}}{d t}=\frac{d \varepsilon_{D}}{d t}+\frac{d \varepsilon_{S}}{d t}=\frac{\sigma}{\eta}+\frac{1}{E} \frac{d \sigma}{d t}
\end{gathered}
$$

where $\mathrm{E}$ is the elastic modulus and $\eta$ the viscosity. When the material is under a constant stress $\sigma_{\text {Total }}$, the generated strain $\varepsilon_{\text {Total }}$ contains two parts, an elastic component $\left(\varepsilon_{\mathrm{S}}\right)$ which occurs instantaneously, and relaxes immediately upon release of the stress; and a viscous component $\left(\varepsilon_{\mathrm{D}}\right)$ that grows with time as long as the stress is applied. The Maxwell model is the simplest description of a viscoelastic liquid, with a single characteristic (relaxation) time given by $\eta / E$.

2, the Kelvin-Voigt model consists of a Newtonian damper and Hookean elastic spring connected in parallel, as shown in Fig. 1.2 (B). It is used to explain the creep behavior of polymers. The model is represented by the first-order differential equation,

$$
\sigma(t)=E \varepsilon(t)+\eta \frac{d \varepsilon(t)}{d t} .
$$

Upon application of a constant stress, the material deforms at a decreasing rate, asymptotically approaching the steady-state strain. The Kelvin model is the simplest decription of a viscoelastic solid, with a single characteristic time given by $\eta / E$.

3, In the Burger model (Fig.1.2(C)), Maxwell and Kelvin models are combined. It gives an example of how the complexity of the model can be increased. Many viscoelastic materials cannot be described with a single characteristic time, and to take that into account, additional (networks of) springs and dashpots can be added, in principle without limitations. However as long as the springs and dashpots cannot be tied to a physical process in the material, the description remains phenomenological.

We remark here that these viscoelastic models only capture a part of the spectrum of possible rheological behaviors. An important assumption is that the deformation of the material is sufficiently small to not significantly modify the structure of the (polymer) network. At large deformations such changes do occur, and the relation between stress and strain becomes nonlinear. 


\subsubsection{Mechanical models for the cell}

To describe the rheology of living cells is more challenging than for engineering materials. One important reason for this is that biological cells, due to their molecular architecture, display heterogeneity. The question then arises how to take that into account: should attempts be made to describe global cell behavior in a simplistic way, e.g. by treating it as an elastic body with a modulus E, or should more sophisticated approaches be attempted? Detailed reviews for mechanical models in living cells can be found in (17-20). In general these models are derived using either a continuum approach or a micro/nano structural approach.

\subsubsection{Continuum models}

Continuum models treat the cell as a body with certain continuum material properties. It includes cortical shell-liquid core models (liquid-drop model) (21$25)$, the solid model $(26,27)$ and the biphasic model $(27-29)$. The liquid-drop model is generally used for suspended cells. The solid model is suited for studying adherent cells; it assumes the cells as homogeneous construct without the distinct cortical layer. In the biphasic model, liquid and solid phases coexist, and their deformations are coupled via equations of motion. This model has been aplied to musculoskeletal cells.

The advantage of continuum approaches is that it is easier and more straightforward to do computations at the cell level. It can provide details about the distribution of stresses and stains induced in the cell which, in turn, can be useful in determining the distribution and transmission of forces to the cytoskeletal and subcellular components. However, it can not provide insight into the detailed molecular deformation and interaction events. For example the structural heterogeneity of cells, or active forces generated within the cells are not captured by these models. Moreover, when the deformations are large enough to modify the structure of cytoskeleton, these models are not valid anymore.

\subsubsection{Micro/nano structural models}

In this section we discuss some of the main models that have been proposed in literature, to describe the passive rheological behavior of living cells.

\section{Tensegrity model}

This classic model was firstly proposed by Ingber (30). It describes the cell as a mechanical framework consisting of opposed elastic struts capable of bearing compressive load. Tensegrity structures consist of elements under tension (ropes) and elements under compression (bars) that produce antagonist forces. In this 
proposed model, actin cables are supposed to play the role of ropes under tension, while microtubules are the compressional elements. It was proven in various studies that prestressed cells can exert traction forces on the substrates when they are crawling (31-33). The importance of this prestress is related with their biological functions such as mechanosensing. This prestress is mainly generated by myosin motors, and can be partially balanced by microtubules but mostly by cell adhesions with extra-cellular matrix and adjacent cells $(11,34-36)$. It is known that microtubule depolymerization can induce tension in cells due to the activation of signal transduction pathways which increases myosin mediated contractility of the cell $(37)$. Brangwynne et al. $(11,34)$ found that microtubules in vivo undergo short-wave bending fluctuations under compressional forces. All these findings have been corroborated with the picture of the tensegrity model.

\section{2. 'Soft-glassy rheology' model (SGR)}

This recent model developed by Sollich et al. (38) predicts a viscoelastic solid in which relaxation process are driven by non-thermal stress fluctuations (e.g. generated by molecular motors in case of living cells). These fluctuations are described with an effective noise temperature. This model has been used to describe a variety of materials (foams, pastes, colloids, emulsions), that have in common that the mechanical moduli are small, the dynamics are slow, and the viscoelasticity shows a weak-power-law frequency dependence (38). This behavior is ascribed to a free-energy landscape, in which many metastable states (local energy wells) occur, and where an exponential distribution of well-depths applies. Since this is also found in systems that are close to a glass transition, the materials showing this behavior have been termed soft glassy materials (SGMs). When SGMs are not exposed to external perturbation, they behave like solid, but upon imposition of sufficient strain or stress, they readily soften and fluidize. Fabry et al. described the frequency dependent rheology of cells with the SGR model (39-41). Recent studies using magnetic twisting cytometry indicated that the SGR model could be valid at intermediate timescales, i.e. larger than the timescales where the dynamics of single filamentous is found (42).

\subsubsection{Active forces}

A limitation of existing models is that they do not distinguish the contributions of intrinsic material properties of the cells or the active forces generated by the intracellular molecular activities, e.g. the contributions of ATP driven processes. Attempts had been made in modeling active force generation and locomotion, e.g. in micropipette studies on neutrophils (43), or in studies on contractile forces in axons (44). However, to what extent motor activity influences the "passive" mechanical response of the cytoskeleton is an unresolved question. 


\subsubsection{Summarizing statements on theoretical modeling}

The present situation in modeling the mechanical behavior of living cells can be summarized as follows: the experimental technique that is used can strongly favor the choice of a particular mechanical model, and the mechanical model that is used can strongly affect the interpreted outcome of the experiment. Continuum and micro/nanostructural approaches both have their drawbacks and both have failed so far, to give a consistent picture of mechanical properties of living cells. Perhaps a hybrid of the two approaches could provide a better approach.

Despite all these discrepancies, some consensus has also been reached on the understanding of cell mechanics: the linear viscoelastic moduli exhibit a power law frequency dependence, cell rheology is scale-free (no characteristic relaxation times determine the dynamics of the cytoskeletal matrix), cells are prestressed, and stiffness and dissipation are altered by stretch (20).

\subsection{Experimental techniques in cell mechanics}

\subsubsection{Overview}

Eventually our understanding of the mechanical behavior of the cell in terms of the molecular constituents of the cytoskeleton and the mechanical models for them, has to come from experiments. Over the past 50 years, many approaches (1, $2,19,45-48$ ) have been developed to measure the mechanical properties (viscoelasticity, deformability) and responses (stiffening/softening over time and length scales) of living cells. Most commonly used approaches used for cell mechanical studies include two subdivisions, 'bottom-up' and 'top-down' methods.

Bottom-up methods use the in vitro reconstitution of 'functional modules' of the cytoskeleton (i.e. cytoskeletal components and/or motor proteins) with the aim to unravel the biological complexity from its physical basis. It builds on the idea of using artificial minimal systems to mimic the mechanical behaviour of the real cells. The advantage of this approach is that the systems are relatively easy to control, and relatively easy to understand from a physical point of view. Several excellent reviews involving bottom-up methods can be found (49, 50). Key findings from these studies are that cross-linked or entangled actin networks show a weak frequency dependent linear visco-elasticity, and a stress-stiffening, that resembles the nonlinear mechanics of living cells.

Top-down methods involve in vivo studies of living cells' mechanical properties or mechanical responses, and were used in this thesis research. The integrated 
mechanical behavior can be directly measured based on single or multiple cells, with or without the application of external mechanical stress. Top-down cell mechanical methods can be classified into active and passive approaches. In this context 'active' refers to the measurement principle (rather than to the cell). In active techniques, the deformation of the cell is controlled via external means, either directly as a deformation or as a force. These quantities are generally well controlled, and the amplitude and timescale can be tuned by the experimentalist.

Active approaches as shown in Table 1.1 are focused on the deformability and elasticity of cells. Here measurements are generally achieved by manipulating either the plasma membrane \& the underneath actin cortex, or the intracellular region, by applying a force/pressure or oscillatory torque and examining the physical response of the cytoskeleton. Each technique has its own advantages and disadvantages regarding the implementation and interpretation and regarding the length- and time-scales they probe. The technique itself is often well-defined, but the cell can introduce considerable complexity. For example in case of large scale deformations, cells can show a complex nonlinear response. Where the nonlinearity comes from can also be unclear, since strain fields can be rather inhomogeneous. Also the combined responses from the cell surface and interior can confound interpretation (47).

Passive approaches include the measurement of rheology-related properties of local regions within the cells or alternatively of whole cells. Also measurements of the cellular response to the external cell matrix without external mechanical perturbation fall into the same category. The characteristics are given in table 1.2. The advantages of applying passive approaches are their low invasiveness and the relatively easy experimental setup. Both methods are not strictly rheological methods. For example particle tracking microrheology in cells can be confounded by difficulties of interpretation, for example related to probe-matrix interaction, heterogeneity of the system, or the interplay of active and passive cytoskeletal dynamics. This will be discussed next. 
Table 1.1, Characteristics of active approaches in cell mechanical studies.

\begin{tabular}{|c|c|c|c|c|c|c|c|}
\hline & Cells & $\begin{array}{l}\text { mechanical } \\
\text { properties }\end{array}$ & physical principle & & $\begin{array}{l}\text { applied } \\
\text { force / } \\
\text { pressure/t } \\
\text { orque } \\
\end{array}$ & $\begin{array}{l}\text { applied } \\
\text { deformation }\end{array}$ & $\begin{array}{l}\text { applied } \\
\text { frequency }\end{array}$ \\
\hline $\begin{array}{l}\text { Microneedle force } \\
\text { transducers }\end{array}$ & suspended single cells & local & $\begin{array}{l}\text { stretching cells with } \\
\text { micropipettes }\end{array}$ & & $0.1-10 \mathrm{nN}$ & $\begin{array}{l}\text { depends on cel } \\
\text { / pipette } \\
\text { diameter ratio }\end{array}$ & $\mathrm{N} / \mathrm{A}$ \\
\hline Micropippette as piration & suspended single cells & local/global & suction & & $\begin{array}{l}0.1- \\
10^{\wedge} 5 \mathrm{~Pa}\end{array}$ & $\begin{array}{l}\text { depends on cel } \\
\text { / pipette } \\
\text { diameter ratio }\end{array}$ & $\mathrm{N} / \mathrm{A}$ \\
\hline Atomic force microscopy & adherent single cells & local & Indentation & & $0.1-10 \mathrm{nN}$ & $\begin{array}{l}<50 \mathrm{~nm} \text { or } \\
<10 \% \text { of cell } \\
\text { height }\end{array}$ & $\begin{array}{l}0.1- \\
300 \mathrm{~Hz}\end{array}$ \\
\hline magnetic tweezer & adherent single cells & local & $\begin{array}{l}\text { twisting attached } \\
\text { microbeads with } \\
\text { magnetic field }\end{array}$ & & $\mathrm{pN}-\mathrm{nN}$ & $1 \mathrm{~nm}-1 \mathrm{um}$ & $\begin{array}{l}0.01- \\
1000 \mathrm{~Hz}\end{array}$ \\
\hline Laser tweezer & adherent single cells & local & $\begin{array}{l}\text { moving or oscillatin } \\
\text { attached microbead } \\
\text { laser tweezer }\end{array}$ & s with & $\sim 10 \mathrm{pN}$ & $10 \mathrm{~nm}$ & $\begin{array}{l}0.01- \\
100 \mathrm{~Hz}\end{array}$ \\
\hline microplate rheometer & adherent single cells & global & $\begin{array}{l}\text { cells stretched betw } \\
\text { two plates, one ind } \\
\text { force, another acts } \\
\text { force transducer }\end{array}$ & & $\begin{array}{l}1 \mathrm{nN}- \\
10 \mathrm{uN}\end{array}$ & 50nm-50um & $\begin{array}{l}0.001- \\
10 \mathrm{~Hz}\end{array}$ \\
\hline cell monolayer shearing & adherent multiple cells & global & $\begin{array}{l}\text { shearing between ty } \\
\text { plates }\end{array}$ & & $>=0.1 \mathrm{~Pa}$ & $>1 \%$ & $\begin{array}{l}0.001- \\
30 \mathrm{~Hz}\end{array}$ \\
\hline \multicolumn{8}{|l|}{ Microfabricated Force } \\
\hline Optical stretcher & suspended single cells & global & $\begin{array}{l}\text { axial deformation by } \\
\text { additive surface for } \\
\text { induced by counter } \\
\text { propagating laser b }\end{array}$ & & $\sim 1 \mathrm{nN}$ & $<0.1$ um & $\mathrm{N} / \mathrm{A}$ \\
\hline \multicolumn{8}{|c|}{$\begin{array}{l}\text { *Ref. (from top to bottom): }(21,22,51,52),(12,53,54),(39,55-57),(58-60),(41,61),(62),(63),(64,65) \\
\text { Table 1.2, Characteristics of passive approaches in cell mechanical studies. }\end{array}$} \\
\hline & $\begin{array}{ll} & \text { me } \\
\text { Cells } & \text { pro } \\
\end{array}$ & $\begin{array}{ll}\text { chanical } \\
\text { perties } & \mathrm{Ph}\end{array}$ & then & Force & & Deformation & Frequency \\
\hline $\begin{array}{l}\text { Elastic substreates as } \\
\text { force transducers }\end{array}$ & $\begin{array}{l}\text { 3D embeded cells } \\
\text { /semi- adherent glot } \\
\text { cells }\end{array}$ & $\begin{array}{l}\mathrm{me} \\
\mathrm{ge}\end{array}$ & $\begin{array}{l}\text { easuring the force } \\
\text { neralted by cells }\end{array}$ & $\begin{array}{l}\text { cell tr } \\
\text { force } \\
\text { 10uN }\end{array}$ & $\begin{array}{l}\text { ractional } \\
\text { up to }\end{array}$ & $\begin{array}{l}\text { substrate } \\
\text { wrinkling, or } \\
\text { deformation of } \\
\text { pillars }\end{array}$ & N/A \\
\hline $\begin{array}{l}\text { Passive particle tracking } \\
\text { microrheology }\end{array}$ & adherent cells & $\begin{array}{l}\text { tra } \\
\mathrm{Br} \\
\text { ord } \\
\mathrm{mi}\end{array}$ & $\begin{array}{l}\text { cing the random } \\
\text { ownian dynamics of } \\
\text { yanelles or } \\
\text { croinjected particles. }\end{array}$ & $\begin{array}{l}\text { Bowr } \\
\text { Brow } \\
\text { depe } \\
\text { proce }\end{array}$ & $\begin{array}{l}\text { nian or non- } \\
\text { Inian (ATP } \\
\text { ndent } \\
\text { esses) }\end{array}$ & $\begin{array}{l}\text { Mean square } \\
\text { displacement, } \\
\text { creep function }\end{array}$ & $\begin{array}{l}0.1-30,000 \\
\mathrm{rad} / \mathrm{s}\end{array}$ \\
\hline
\end{tabular}

*Ref. (from top to bottom): (31-33, 66, 67), (68-75)

\subsubsection{Particle Tracking}

Tracking the motions of particles inside living cells can be seen as a mechanical method in the sense that the motion of intracellular particles is the consequence of driving forces and resistive forces operating within the cell. Through quantification of the driving force, the viscoelastic resistance can be inferred from the particle motion. So far this principle has mostly been applied to engineering materials, for which the driving forces can be assumed to be thermal (in passive experiments). Since cells are much more complex, we discuss the aspects of particle tracking microrheology and intracellular particle tracking in separate sections below. 


\subsubsection{Microrheology}

There has been a long history of using colloidal probe particles as tracers in study of viscoelastic materials. The first finding enabling the later application of particle tracking microrheology was by a Scottish biologist, Robert Brown (1827) who found that pollen grains suspended in water were moving incessantly and erratically. In 1905, Albert Einstein analyzed Brownian motion theoretically as $D=\mu_{P} k_{B} T$, where $\mathrm{D}$ is the diffusion coefficient, $\mu_{\mathrm{P}}$ is the diffusivity of the particle, $\mathrm{k}_{\mathrm{B}}$ is Boltzmann's constant, and $\mathrm{T}$ is the absolute temperature. Together with Stokes' law discovered by George Gabriel Stokes in 1851, $F_{d}=6 \pi \eta R v$ which describes the drag force $F_{d}$ exerted on spherical objects with radius $R$, moving through a viscous liquid with a velocity $v$ (small enough to keep the flow laminar) in a fluid with viscosity $\eta$, this gives the Stokes-Einstein relation,

$$
D=\frac{k_{B} T}{6 \pi \eta R} .
$$

This work was extended by Jean Baptiste Perrin (1948) who demonstrated that the mean-square-displacement (MSD) of gutta-percha particles in water is directly proportional to time, with a proportionality constant that describes the frictional dissipation in the liquid surrounding the particles. Combining these elementary findings leads to the well-known expression for the MSD of a diffusive system: the MSD of a particle in $\mathrm{n}$ dimensions depends linearly on time (t) with a proportionality constant $\mathrm{D}$ defined as the diffusive coefficient for translational motion:

$$
\left\langle r^{2}\right\rangle=2 n D t
$$

with $\left\langle r^{2}\right\rangle=\left\langle r_{x}{ }^{2}\right\rangle+\left\langle r_{y}{ }^{2}\right\rangle$ the mean square fluctuations in the $\mathrm{x}$ and $\mathrm{y}$ directions (for $\mathrm{n}=2$ ).

When particles diffuse through a viscoelastic medium or are transported in a non-diffusive manner, $\left\langle r^{2}\right\rangle$ becomes nonlinear with time and can be formally described with a power law, $\left\langle r^{2}\right\rangle=2 n C t^{\alpha}$ where $\alpha$ is the diffusive exponent and $\mathrm{C}$ a constant. In a simple Newtonian liquid (e.g. water or glycerol), $\alpha$ is equal to 1 in formula (4). The viscosity of the fluid is thus calculated by combining equation (3) and (4),

$$
\eta=\frac{2 n k_{B} T}{6 \pi R} \frac{t}{\left\langle r^{2}\right\rangle} .
$$

In a simple Hookean material, $\alpha$ is equal to 0 , and hence the MSD is independent of lagtime t. The elastic shear modulus can then be calculated as 


$$
G=\frac{2 n k_{B} T}{6 \pi R\left\langle r^{2}\right\rangle} .
$$

The appearance of $\mathrm{k}_{\mathrm{B}} \mathrm{T}$ in the enumerator of both expressions underlines that the motions of the particles are assumed to be driven by thermal collisions from the surrounding molecules. This is why this analysis method is called passive microrheology. In a passive complex fluid where both viscous and elastic elements exist, a sub-diffusive behavior is observed, $0<\alpha<1$. In a non-passive system, for example living cells with motor-protein-assisted-motion (76-78), one can find $1<\alpha<2$, which is called superdiffusive behavior. For ideal ballistic motion in which particles move unidirectionally, $\alpha=2$. An overview of these behaviors can be found in Fig.1.3.

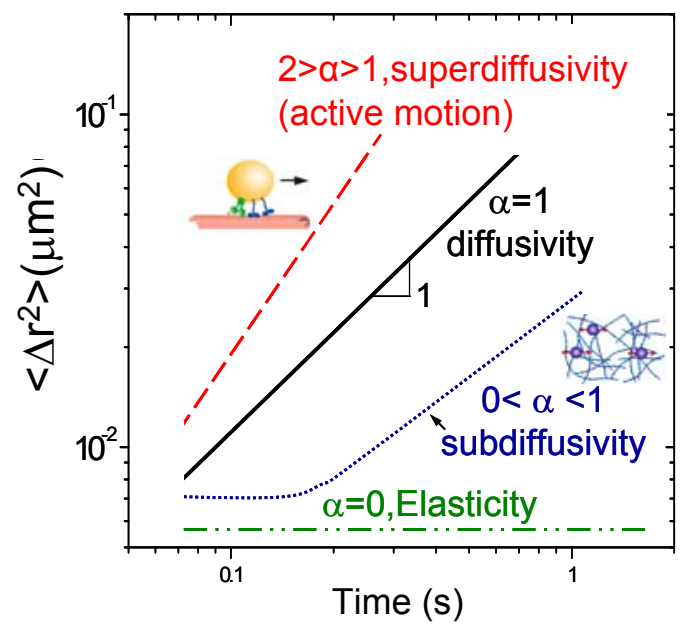

Fig.1.3, Types of MSDs encountered in PTR.

Quantitative analysis of subdiffusive motion can allow the calculation of the rheological properties of the material. However, a certain challenge lies in how to transfer from the mean square displacement as a function of time, obtained from subdiffusive motion of particles, to the linear viscoelastic spectra as a function of frequency. The linear viscoelasticity is normally expressed in the storage modulus ( $\left.G^{\prime}\right)$ and the loss modulus (G'). In inert soft materials, the time dependence of thermally driven probe particle displacements can be interpreted in the framework of the Generalized Stokes-Einstein Relation (GSER) (79) to extract the frequency dependent viscoelastic behavior of the material,

$$
\widetilde{D}(s)=\frac{k T}{6 \pi a s \tilde{\eta}_{s}}
$$


where $a$ is the radius of the probe sphere, $\tilde{\eta}_{s}$ is the Laplace transformed frequency dependent viscosity, $\widetilde{D}(s)$ is the Laplace transformed frequency dependent diffusion coefficient and $\mathrm{s}$ is the Laplace frequency.

However, besides some technical issues of how to transform from time domain to frequency domain (79), also several physical conditions are critical to validate this conversion: (i) the probes should not show specific binding to or modification of the local microstructure of the medium in which they are embedded, (ii) they are bigger than the mesh size of the structure that is responsible for the rheology, (iii) the driving force for the probe particles is thermal fluctuation, (iv) "no-slip" boundary conditions should apply at the surface of the probe particle, and (v) the medium to be probed is homogeneous in order to extract the bulk rheological properties of the material. These criteria are relatively easier to satisfy in inert engineering materials, formulated from a small number of compounds. However, in living cells due to their biological complexity and structural heterogeneity, experimental verification of the fulfillment of these criteria is needed and can be far from straightforward.

In addition to this version of particle tracking microrheology (also known as 1PMR) which correlates single particle MSDs as a function of time to the rheological properties of the material, another technique called two-point microrheology (2-PMR) was presented in $2000(80,81)$. In this method, the correlated movements of pairs of neighboring particles are used to measure the relative viscoelastic response on the timescale of the single probe particles. In contrast to the conventional way of computing MSDs, the effective single particle MSDs obtained from 2-PMR are insensitive to material heterogeneities, (variations in) tracer size and boundary conditions at the surface of the probes. In 2-PMR, the displacement-cross-correlation functions consistently depend on tracer pair separation $r$ as $\sim 1 / r$.

\subsubsection{Intracellular particle tracking (IPT)}

Many studies aimed at quantifying the mechanical properties of living cells have used passive particle tracking microrheology. Three kinds of probes have been attempted in this kind of studies: phagocytosed or endocytosed particles, endogenous granules (EGs) and microinjected particles. Motions of phagocytosed or endocytosed particles are normally rather complex, varying from subdiffusive to superdiffusive (48). Their particle dynamics is generally driven directly by molecular motors, and/or indirectly via the movements of cytoskeletal filaments. This makes these probes unsuitable for measuring rheological properties from their MSDs. 
EGs and microinjected particles have been more widely used to examine intracellular rheology. Endogenous granules $(68,82)$ are naturally present cellular organelles (usually lipid droplets and mitochondria) that are associated with specific biological functions in living cells. The use of EGs as intracellular probes is motivated by the (potential) capability to measure cellular mechanical properties with minimal disruption of the intracellular structure. However comparison of MSDs obtained with 1-PMR and 2-PMR has demonstrated that also in the dynamics of EGs, superdiffusivity can occur (68). To create a passive cellular environment, ATP depletion was applied in one study (68), to disrupt the non-Brownian contributions to EGs motion.

Alternatively, probes with selectable size and surface chemistry have also been injected into cells either by a ballistic gun or a microinjector (70-74). In certain cells, microinjected and ballistically injected particles (BIPs) have been found to behave similarly (70). BIPs have the advantage of the introduction of many particles per cell, simultaneously in many cells. Prior studies have noted that polystyrene latex particles, with either (negatively charged) carboxyl groups (73) or a coating of polyethylene glycol chains (83) on the outer surface, approach the ideal 'inert probe chemistry', although it has also been remarked that a definitive establishment of probe-matrix interactions has not yet been reached. The mesh size of the cytoskeletal network has been addressed in several studies as either 50 $\mathrm{nm}$ in the perinuclear area (73) or $100 \mathrm{~nm}$ in cellular cortical actin networks (84). A comparison for the suitability of EGs and BIPs as intra-cellular rheological probes is given in table 1.3. This overview presents the generally accepted current view on IPT. Views on the physical interpretation of MSDs have evolved considerably in the past 10 years. We see that most of the criteria are not a priori fulfilled to validate a direct conversion from MSDs to viscoelastic moduli. If a passive intracellular environment could be indeed reached (as suggested in (68)), then this would allow rheological studies of living cells. In other cases still valuable information could be obtained from intracellular MSDs, i.e. probematrix interactions or the mechanics of cytoskeletal networks. 
Table 1.3, Evaluation of the suitability of using EGs and microinjected particles as rheological probes in living cells.

\begin{tabular}{|l|l|l|}
\hline & EGs & Microinjected particles \\
\hline $\begin{array}{l}\text { Probe } \\
\text { surface } \\
\text { chemistry }\end{array}$ & $\begin{array}{l}\text { Unknown (membrane } \\
\text { enclosed) }\end{array}$ & $\begin{array}{l}\text { Controllable, usually (-) charged } \\
\text { carboxylated or polyethylene } \\
\text { glycol (PEG) coated particles }\end{array}$ \\
\hline Probe size & Variable, mean size $\sim 500 \mathrm{~nm}$ & Known (commonly used 100nm) \\
\hline Driving force & $\begin{array}{l}\text { Motor-driven active motion } \\
\text { exists }\end{array}$ & $\begin{array}{l}\text { Unknown (believed to be thermal } \\
\text { in most studies) }\end{array}$ \\
\hline $\begin{array}{l}\text { No-slip } \\
\text { boundary } \\
\text { condition }\end{array}$ & Unknown & Unknown \\
\hline References & $(68,85)$ & $(70-74)$ \\
\hline
\end{tabular}

\subsubsection{Atomic Force Microscopy}

Atomic force microscopy (AFM) has also been used as a tool for measuring the (visco-) elastic properties of living cells at the nanoscale $(12,53,54)$. The basic principle has been described elsewhere (86) and is briefly summarized here. The central element is a cantilever spring with a tip attached to its free end (see Fig. 1.4) When the AFM tip contacts the cell surface, due to the attractive or repulsive forces between the tip and the cell surface, the angular deflection of the cantilever will change. This is detected via a laser beam that is reflected against the back side of the cantilever. The location of this spot is then constantly monitored by a position-sensitive photodetector consisting of quadrant photodetector. The difference between two photodiode signals then indicates the bending or torsion of the cantilever. These deflection signals can be either used to extract the information about surface topography of the cells or about mechanical properties of the living cell. The force $F$ applied by the AFM can be obtained via Hooke's law, $F=K_{c} d$, where $K_{c}$ is the spring constant of cantilever, and $d$ the deflection of cantilever end. This force is generally balanced by the response force originating from the material, which is often expressed as a function of indentation depth. The indentation depth is obtained by subtracting the deflection from the displacement $Z$ of the cantilever base. 


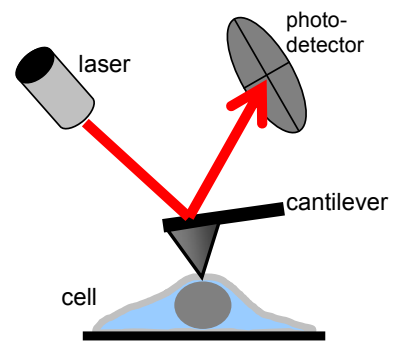

Fig.1.4, Schematic view of an AFM measurement on a living cell.

\subsubsection{Four commonly used AFM modes in cell mechanical studies}

In Fig. 1.5, the most commonly used modes in AFM are illustrated. Each of these will be discussed below.

1, Force-distance mode (Fig. 1.5.1) is the most commonly used measurement of elastic properties of the cell's membrane and underlying cytoskeleton (87). The loading force of the cantilever can be plotted as a function of the depth of indentation as the AFM tip is pushed against the cell surface. The Young's modulus can then be quantified using various models. The most commonly used model is the Hertz model (1881) (87). If the tip is approximated by a sphere with the radius $R$, and the cell is approximated by a thick slab, then the force on the cantilever is approximated by

$$
F=\frac{3 \sqrt{R}}{4} \frac{E}{1-v^{2}} \delta^{3 / 2},
$$

where $\delta$ is the depth of the indentation, and $E$ and $v$ are respectively the Young's modulus and the Poisson ratio of the cell. Bilodeau (1992) has extended this formula to a four-sided pyramidal tip:

$$
F=\frac{3}{4} \tan (\alpha) \frac{E}{1-v^{2}} \delta^{2},
$$

where $\alpha$ is the half opening angle of the AFM tip. Which of the two equations is most accurate depends on the depth of indentation. A typical tip has a height of several microns and a radius of curvature at its apex of 25-50 nm. Hysteresis (i.e. the approach and retraction curves do not coincide, see Fig. 1.5.3) often appears in force-distance measurements on living cells. This effect, indicating that energy dissipated during the approach and retraction could be due to a (partly) viscous deformation of the cell. However, the magnitude of the corresponding viscosity can be not resolved from this measurement. 
2, Creep mode (Fig.1.5.2) is the measurement of the displacement of the cell surface as a function of time at a constant loading force, which is controlled by the AFM feedback circuit. Also the creep response depends on the viscoelastic properties of the cell. Wu et al (88) have studied in detail the relationship between the viscoelastic properties and the cytoskeletal architecture of L929 cells with and without drugs that disrupt the cytoskeleton.

3, Force modulation mode (Fig.1.5.3) is the measurement of the dynamic response of the loading force with respect to an external periodic strain. This mode allows measuring the frequency response of both the storage and loss moduli of living cells, after a correction for tip-cell contact geometry and hydrodynamic (i.e. viscous) drag. This mode has been applied to study human lung epithelial cells (89) and air smooth muscle cells (90) at frequencies of 0.1 $100 \mathrm{~Hz}$.

4, Stress relaxation mode (Fig.1.5.4) is the measurement of the time dependence of the loading force while the cantilever base is held fixed. Studies done by Okajima $(91,92)$ reported that the decay of loading force observed on the HepG2 cells should be attributed to the viscoelastic properties of these cells. The relaxation process appeared to involve timescales of $<1.5 \mathrm{~s}$.

1

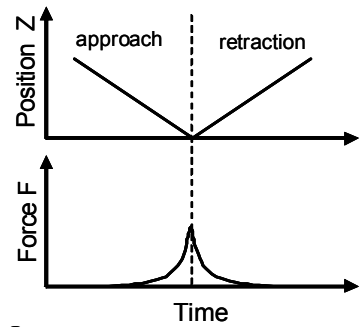

2

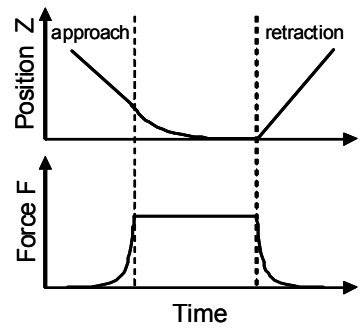

3

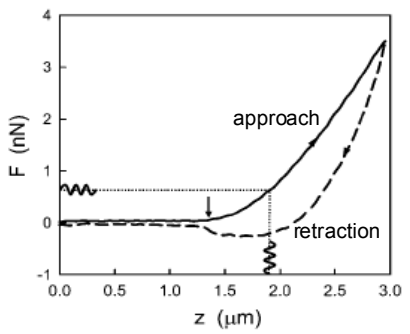

4

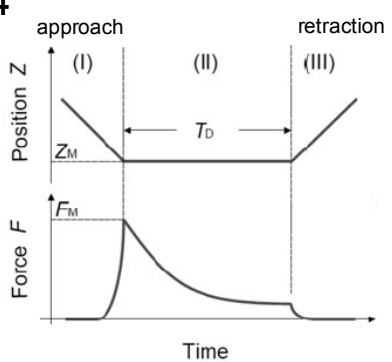

Fig.1.5, Schematic view of four commonly used AFM measurements in cell mechanical studies. 1.4.3 and 1.4.4 are reprinted from (89)and (91)respectively. 


\subsubsection{Main issues in AFM measurements on living cells}

In spite of its sophistication, AFM sometimes still needs to be considered as a semi-quantitative technique. This applies in particular to measurements on living cells. The most important reasons are given below.

\section{Spatial heterogeneity of the cell surface}

The apparent elastic modulus can vary dramatically for different regions on the cell surface. For example, the cell nuclear region, bulky membrane region and thin membrane region.

\section{Determination of the contact point}

Because cells are very soft, the precise contact point where the tip starts to indent on the cells is very difficult to determine. The large uncertainty in the contact point can lead to significant errors in the estimation of the indentation depth and elastic moduli (93).

\section{Models to calculate elasticity of the cells}

In force-distance mode, the most commonly used model is the Hertz model. However, living cells are anisotropic and not necessarily behave elastically without a viscous component. In addition, the Hertz model is not appropriate where adhesive forces are present. The Poisson ratio cannot be measured in the indentation experiment, although in practice 0.5 (the case of an incompressible body) is usually assigned. Other models are, for example theories of elastic shells $(94,95)$ and finite element models $(53,96)$. The elastic modulus calculated using different models can vary by more than one order of magnitude (96).

\section{Characteristics of the AFM cantilever}

The choice of the cantilever spring constant and accurate calibration hereof is critical to quantitative measurements. The stiffness of the cantilever should be comparable to the effective elastic stiffness of the cell because otherwise either the deflection becomes too low (for a too stiff cantilever), or alternatively the attractive or repulsive, non-contact forces between the tip and the sample could obscure the definition of the contact point (for too soft cantilever).

\section{Other}

Other uncertainties include the hydrodynamic drag force, the effect of finite sample (i.e. cell) thickness, and the precise geometry of AFM tip.

In conclusion, AFM force measurements on single cells should be carefully processed and interpreted taking into account the limitations of AFM. Many of the mentioned issues are less serious when semi-quantitative comparative studies are done, e.g. when studying the effect of a drug or when comparing healthy and diseased cells. 


\subsection{This thesis}

At the start of this thesis research, the first intracellular particle tracking (IPT) studies had been reported in literature, but a lot was still unclear. In several papers (70-74), IPT had been presented as a microrheological method, allowing the measurement of viscoelastic moduli of the cell interior, at the timescales (typically 0.1-30 s) accessible to video microscopy. However, other papers had demonstrated violations of the fluctuation-dissipation theorem that underlies the GSER used in IPT. Also ATP depletion had been applied to cells, with apparent success, since a universal behavior was found for the calculated viscoelastic moduli. This indicated that there were many perspectives but also many uncertainties associated with IPT. Some issues had to be addressed or readdressed in particular. All of these were centered on the question, which information can be extracted from an intracellular MSDs. Separate issues related to this central question will be treated in the individual chapters that follow.

Chapter 2 discusses in detail the materials and experimental methods that were used in this thesis work.

Chapter 3 addresses sampling issues in IPT, in relation to the intracellular heterogeneity. Strong variations between MSDs of individual particles present at different locations in the same cell imply that getting an average MSD that represents the cell as a whole, is not always trivial. Especially if the number of particles per cell is limited, or if there would be bias for the occurrence of particles in certain regions, then the "error bar" in the MSD corresponding to the cell could become rather large.

A related question is, what kind of information could be obtained from the MSDs of the individual particles. In a complex dynamic body as a living cell, the dynamic behavior of the cytoskeleton could show a palette of amplitudes and (sub, super) diffusive behaviors. Also a changeover from one behavior to another might take place within the time that a particle is tracked. This implies that ensembles of intracellular particles could reveal information about intracellular dynamics beyond the level of the average MSD function. The findings in this chapter will be used as a basis for the analysis of cell-averaged MSDs as well as distributions for dynamic properties like the amplitude and exponent, as will be used in the subsequent chapters.

Besides these statistical aspects, also questions about the physical meaning of intracellular MSDs will be considered. In an early experiment, both EGs and 
BIPs were studied inside the same cell, and the (average) MSDs looked strongly different. This raised a series of questions. Could one of the two (or both) reveal intracellular rheology? Or alternatively, could evidence be found for ATP dependent driving forces? Which differences in micro environment (cytoskeletal components, structure, probe-matrix interaction) could be responsible for the different MSDs? These questions were addressed in two related studies.

Chapter 4 reports about a study into the dynamics of EGs in human endothelial cells. To evaluate the possible microenvironment of EGs and possible driving mechanism of EGs motion, various drug interventions targeted on different cytoskeletal components and active forces were applied. Chapter 5 describes a similar study on the dynamics of BIPs in the same type of endothelial cells.

In yet another angle of approach, intracellular MSDs could also be seen as a signature of the health state of the cell. The use of MSDs for this purpose had hardly been explored in literature, but could have great potential for analyzing small amounts of cell material for diagnostic or prognostic purposes. In chapter 6, the dynamics of both BIPs and EGs were studied in two pairs of tumor cells with different metastatic potentials. Also elasticity measurements with AFM were done on the same cell types, to corroborate the IPT results or to assess the differences with the findings with IPT. 


\section{References:}

1. C. R. Ethier and C.A.Simmons, Introductory biomechanics: from cells to organisms, Cambridge University Press, Cambridge, UK (2007).

2. Y. Wang and D.E.Discher, Methods in cell biology: cell mechanics, Elsevier Inc., Califonia, USA (2007).

3. D. E. Discher, P. Janmey and Y. L. Wang, "Tissue cells feel and respond to the stiffness of their substrate," Science 310, 1139-1142 (2005)

4. P. A. Janmey and D. A. Weitz, "Dealing with mechanics: mechanisms of force transduction in cells," Trends in biochemical sciences 29(7), 364370 (2004)

5. H. Huang, R. D. Kamm and R. T. Lee, "Cell mechanics and mechanotransduction: pathways, probes, and physiology," Am J Physiol Cell Physiol 287, C1-C11 (2004)

6. D. E. Ingber, "Cellular mechanotransduction:putting all the pieces together again," FASEB J. 20, 811-827 (2006)

7. S. Suresh, "Biomechanics and biophysics of cancer cells," Acta Biomaterialia 3, 413-438 (2007)

8. S. Suresh, J. Spatz, J. P. Mills, A. Micoulet, M. Dao, C. T. Lim, M. Beil and T. Seufferlein, "Connection between single-cell biomechanics and human disease states: gastrointestinal cancer and malaria," Acta Biomaterialia 1, 15-30 (2005)

9. G. Y. H. Lee and C.T.Lim, "Biomechanics approaches to studying human diseases," Trends in Biotechnology 25(3), 111-118 (2007)

10. B. Alberts, A. Johnson, J. Lewis, M. Raff, K. Roberts and P. Walter, Molecular biology of the cell, Garland Science, New York, USA (2002).

11. C. P. Brangwynne, F.C.Mackintosh, S.Kumar, N.A.Geisse, J.Talbot and e. a. L.Mahadevan, "Microtubules can bear enhanced compressive loads in living cells because of lateral reinforcement," J. Cell Biol. 173, 733-741 (2006)

12. S. Sivaramakrishnan, J. V. DeGiulio, L. Lorand, R. D. Goldman and K. M. Ridge, "Micromechanical properties of keratin intermediate filament networks," PNAS 105, 889-894 (2008)

13. V. Breedveld and D. J. Pine, "Microrheology as a tool for highthroughput screening," Materials science J. 38, 4461-4470 (2003)

14. F. C. Mackintosh and C.F. Schmidt, "Microrheology," Current Opinion in Colloid \& Interface Science 4, 300-307 (1999)

15. C. W. Macosko, Rheology: Principles, Measurements and Applications, John Wiley \& Sons, New York (1994). 
16. T. A. Waigh, "Microrheology of complex fluids," Rep.Prog.Phys. 68, 685-742 (2005)

17. K. E. Kasza, A. C. Rowat, J. Liu, T. E. Angelini, C. P. Brangwynne, G. H. Koenderink and D. A. Weitz, "The cell as a material," Curr. Opin. in Cell Biol. 19, 101-107 (2007)

18. C. T. Lim, "Mechanical models for living cells - a review," J.Biomech. 39, 95-216 (2006)

19. P. A. Pullarkat, P. A. Fernandez and A. Ott, "Rheological properties of the eukaryotic cell cytoskeleton," Physics Reports 449, 29-53 (2007)

20. X. Trepat, G. L. Lenormand and J. J. Fredberg, "Universality in cell mechanics," Soft matter 4, 1750-1759 (2008)

21. E. Evans and B. Kukan, "Passive material behavior of granulocytes based on large deformation and recovery after deformation tests," Blood 64(5), 1028-1035 (1984)

22. E. Evans and A. Yeung, "Apparent viscosity and cortical tension of blood granulocytes determined by micropipet aspiration," Biophys. J. 56(1), 151-160 (1989)

23. C. Dong, R. Skalak, K. L. Sung, G. W. Schmid-Schonbein and S. Chien, "Passive deformation analysis of human leukocytes," J. Biomech. Eng. (1988)

24. G. W. Schmid-Schonbein, Y. Y. Shih and S. Chien, "Morphometry of human leukocytes," Blood 56(5), 866-875 (1980)

25. M. A. Tsai, R. S. Frank and R. E. Waugh, "Passive mechanical behavior of human neutrophils: power-law fluid," Biophys. J. 65(5), 2078-2088 (1993)

26. W. R. Jones, P. H. Ting-beall, G. M. Lee, S. S. Kelley, R. M. Hochmuth and F. Guilak, "Alterations in the Young's modulus and volumetric properties of chondrocytes isolated from normal and osteoarthritis human cartilage," J. Biomech. 32(2), 119-127 (1999)

27. D. P. Theret, M. J. Levesque, M. Sato, R. M. Nerem and L. T. Wheeler, "The appliction of a homogeneous half-space model in the analysis of endothelial cell micropipette measurements," J. Biomech. Eng. 110(3), 190-199 (1988)

28. A. C. Schieh and K. A. Athanasiou, "Biomechanics of single chondrocytes and osteoarthritis," Crit. Rev. Biomed. Eng. 30(4-6), 3-0$343(2002)$

29. A. C. Schieh and K. A. Athanasiou, "Principles of cell mechanics for cartilage tissue engineering," Ann. Biomed. Eng. 31(1), 1-11 (2003)

30. D. E. Ingber, "Cellular tensegrity: Defining new rules of biological design that govern the cytoskeleton," J. Cell Sci. 104, 613 (1993) 
31. M. Demco, T. Oliver, A. Ishihara and K. Jacobson, "Imaging the traction stresses exerted by locomoting cells with the elastic substratum method," Biophys. J. 70, 2008-2022 (1996)

32. A. K. Harris, P. Wild and D. Stopak, "Silicone rubber substrata: a new wrinkle in the study of cell locomotion," Science 208(4440), 177-179 (1980)

33. J. L. Tan, J. Tien, D. M. Pirone, D. S. Gray, K. Bhadriraju and C. S. Chen, "Cells lying on a bed of microneedles: an apporach to isolate mechnical force," Proc. Natl. Acad. Sci. USA. 100(4), 1484-1489 (2003)

34. C. P. Brangwynne, F. C. MacKintosh and D. A. Weitz, "Force fluctuations and polymerization dynamics of intracellular microtubules," Proc. Natl. Acad. Sci. USA. 104, 16128-16133 (2007)

35. S. Kumar, I. Z. Maxwell, A. Heisterkamp, T. R. Polte, T. P. Lele, M. Salanga, E. Mazur and D. E. Ingber, "Viscoelastic retraction of single living stress fibers and its impact on cell shape, cytoskeletal organization, and extracellular matrix mechanics," Biophys. J. 90, 3762-3773 (2006)

36. D. Stamenovic, B. Suki, B. Fabry, N. Wang and J. J. Fredberg, "Rheology of airway smooth muscle cells is associated with cytoskeletal contractile stress," J. Appl. Physiol. 96, 1600-1605 (2004)

37. D. E. Ingber, S. R. Heidemann, P. Lamoureux and R. E. Buxbaum, "Opposing views on tensegrity as a structural framework for understanding cell mechanics," J. Appl. Physiol. 89,1663-1678 (2000)

38. P. Sollich, F. Lequeux, P. Hébraud and M. E. Cates, "Rheology of soft glassy materials," Phys. Rev. Lett. 78, 2020 - 2023 (1997)

39. B. Fabry, G. N. Maksym, J. P. Butler, M. Glogauer, D. Davajas and J. J. Fredberg, "Scaling the microrheology of living cells," Phys. Rev. Lett. 87(14), 148102 (2001)

40. P. Bursac, G. Lenormand, B. Fabry, M. Oliver, D. A. Weitz, V. Viasnoff, J. P. Butler and J. J. Fredberg, "Cytoskeletal remodelling and slow dynamics in the living cell," Nat. Mater. 4, 557-561 (2005)

41. N. Desprat, A.Richert, J.Simeon and A.Asnacios, "Creep function of a single living cell," Biophys. J. 88, 2224-2233 (2005)

42. L. Deng, X. Trepat, J. P. Butler, E. Millet, K. G. Morgan, D. A. Weitz and J. J. Fredberg, "Fast and slow dynamics of the cytoskeleton," Nat. Mater. 5, 636-640 (2006)

43. M. Herant, W. A. Marganski and M. Dembo, "The mechanics of neutrophils: synthetic modeling of three experiments," Biophys. J. 84, 3389-3413 (2003)

44. R. Bernal, P. A. Pullarkat and M. Francisco, "Mechanical Properties of Axons," Phys. Rev. Lett. 99, 018301-018305 (2007) 
45. G. Y. H. Lee and C.T.Lim, "Biomechanics approaches to studying human diseases," Trends Biotechnol. 25, 111-118 (2007)

46. A. E. Pelling and M. A. Horton, "An historical perspective on cell mechanics," Eur. J. Physiol. 456, 3-12 (2008)

47. K. J. Van Vliet, G. Bao and S. Suresh, "The biomechanics toobox: experimental approaches for living cells and biomolecules," Acta materialia 51, 5881-5905 (2003)

48. D. Weihs, T. G. Mason and M. A. Teitell, "Bio-microrheology: A frontier in microrheology," Biop. J. 91, 4296-4305 (2006)

49. A. R. Bausch and K. Kroy, "A bottom-up approach to cell mechanics," Nature Physics 2, 231-238 (2006)

50. K. Kroy, "Elasticity,dynamics and relaxation in bioploymer networks," Curr. Opin. in Colloid \& Interface Science 11, 56-64 (2006)

51. E. Evans, "New membrane concept applied to the analysis of fluid shearand micropipette-deformed red blood cells," Biophys. J. 13, 941-954 (1973)

52. M. Sato, M. J. Levesque and R. M. Nerem, "An application of the micropipette technique to the measurement of the mechanical properties of cultured bovine aortic endothelial cells," Trans. ASME J. Biomech. Eng. 109, 27-34 (1987)

53. G. T. Charras and M. A. Horton, "Single cell mechanotransduction and its modulation analyzed by atomic force microscope indentation," Biophys. J. 82, 2970-2981 (2002)

54. G. T. Charras, P. P. Lehenkari and M. A. Horton, "Atomic force microscopy can be used to mechanically stimulate osteoblasts and evaluate cellular strain distributions," Ultramicroscopy 86, 85-95 (2001)

55. A. R. Bausch, F. Ziemann, A. A. Boulbitch, K. Jacobson and E. Sackmann, "Local measurements of viscoelastic parameters of adherent cell surfaces by magnetic bead microrheometry," Biophys. J. 75, 20382049 (1998)

56. G. N. Maksym, B. Fabry, J. P. Butler, D. Navajas, D. J. Tschumperlin, J. D. Laporte and J. J. Fredberg, "Mechanical properties of cultured human airway smooth muscle cells from 0.05 to $0.4 \mathrm{~Hz}$," J. Appl. Physiol. 89, 1619-1632 (2000)

57. P. A. Valberg and D. F. Albertini, "Cytoplasmic motions, rheology, and structure probed by a novel magnetic particle method," J. Cell Biol. 101, 130-140 (1985)

58. A. Ashkin, "Optical trapping and manipulation of neutral particles using lasers," Proc. Natl. Acad. Sci. USA. 94, 4853 (1997) 
59. J. P. Mills, L. Qie, M. Dao, C. T. Lim and S. Suresh, "Nonlinear elastic and viscoelastic deformations of the human red blood cell with optical tweezers," Mol. Cell Biol. 1, 169 (2004)

60. M. P. Sheetz, Laser Tweezers in Cell Biology, Academic Press, San Diego, USA (1998).

61. P. Fenandez, P. A. Pullarkat and A. Ott, "A master relation defines the nonlinear viscoelasticiy of single fibroblasts," Biophys. J. 90, 3796-3805 (2006)

62. L. Eichinger, X. B. Köppel, A. A. Noegel, M. Schleicher, M. Schliwa, K. Weijer, W. Witke and P. A. Janmey, "Mechanical perturbation elicits a phenotypic difference between dictyostelium wild-type cells and cytoskeletal mutants," Biophys. J. 70, 1054 (1996)

63. S. Yang and M. T. A. Saif, "Microfabricated Force Sensors in the Study of Cell Mechanical Response," Experimental Mechanics 49, 135-151 (2009)

64. J. Guck, R. Ananthakrishnan, H. Mahmood, T. J. Moon, C. C. Cunningham and J. Käs, "The optical stretcher: a novel laser tool to micromanipulate cells," Biophys. J. 81, 767-784 (2001)

65. F. Wottawah, S. Schinkinger, B. Lincoln, R. Ananthakrishnan, M. Romeyke, J. Guck and J. Käs, "Optical rheology of biological cells," Phys. Rev. Lett. 94, 098103 (2005)

66. C. G. Galbraith and M. P. Sheetz, "A micromachined device provides a new bend on fibroblast traction forces," Proc. Natl. Acad. Sci. USA. 94, 9114-9118 (1997)

67. J. Lee, M. Leonard, T. Oliver, A. Ishihara and K. Jacobson, "Traction forces generated by locomoting keratocytes," J. Cell Biol. 127, 19571964 (1994)

68. B. D. Hoffman, G. Massiera, K. M. V. Citters and J. C. Crocker, "The consensus mechanics of cultured mammalian cells," Proc. Natl. Acad. Sci. USA.103, 10259-10264 (2006)

69. A. W. C. Lau, B. D. Hoffman, A. Davies, J. C. Crocker and T. C. Lubensky, "Microrheology, stress fluctuations, and active behavior of living cells," Phys. Rev. Lett. 91, 198101 (2003)

70. P. Panorchan, J. S. Lee, B. R. Daniels, T. P. Kole, Y. Tseng and D. Wirtz, "Probing cellular mechanical responses to stimuli using ballistic intracellular nanorheology," Meth. Cell. Biol. 83, 115-140 (2006)

71. P. Panorchan, J. S. Lee, T. P. Kole, Y. Tseng and D. Wirtz, "Microrheology and ROCK Signaling of human Endothelial cells embedded in a 3D matrix," Biophys. J. 91, 3499-3507 (2006) 
72. Y. Tseng, T. P. Kole, S. H. J. Lee and D. Wirtz, "Local dynamics and viscoelastic properties of cell biological systems," Curr. Opin. in Colloid \& Interface Science 7, 210-217 (2002)

73. Y. Tseng, T. P. Kole and D. Wirtz, "Micromechanical mapping of live cells by multiple-particle-tracking microrheology," Biophys. J. 83, 3162$3176(2002)$

74. Y. Tseng, J. S. Lee, T. P. Kole, I. Jiang and D. Wirtz, "Microorganization and visco-elasticity of the interphase nucleus revealed by particle nanotracking," J. Cell Sci. 117, 2159-2167 (2004)

75. S. Yamada, D. Wirtz and S. C. Kuo, "Mechanics of living cells measured by laser tracking microrheology," Biophys. J. 78, 1736-1747 (2000)

76. A. Caspi, R. Granek and M. Elbaum, "Enhanced diffusion in active intracellular transport," Phys. Rev. Lett. 85, 5655-5658 (2000)

77. C. Pangarkar, A. T. Dinh and S. Mitragotri, "Dynamics and spatial organization of endosmomes in mammalian cells," Phys. Rev. Lett. 95, $158101(2005)$

78. J. Snider, F. Lin, N. Zahedi, V. Rodionov, C. C. Yu and S. P. Gross, "Intracellular actin-based transport: How far you go depends on how often you switch," Proc. Natl. Acad. Sci. USA. 101, 13204-13209 (2004)

79. T. G. Mason and D. A. Weitz, "Optical Measurements Of FrequencyDependent Linear Viscoelastic Moduli Of Complex Fluids," Phys. Rev. Lett. 74,1250-1253 (1995)

80. J. C. Crocker, M. T. Valentine, E. R. Weeks, T. Gisler, P. D. Kaplan, A. G. Yodh and D. A. Weitz, "Two-point microrheology of inhomogeneous soft materials," Phys. Rev. Lett. 85, 888-891 (2000)

81. A. J. Levine and T. C. Lubensky, "One and two particle microrheology," Phys. Rev. Lett. 85(8), 1774-1777 (2000)

82. A. Lau, B. D. Hoffman, A. Davies, J. C. Crocker and T. C. Lubensky, "Microrheology, stress fluctuations, and active behavior of living cells," Phys. Rev. Lett. 91, 198101 (2003)

83. P. Panorchan, D. Wirtz and Y. Tseng, "Structure-function relationship of biological gels revealed by multiple-particle tracking and differential interference contrast microscopy: The case of human lamin networks," Phys. Rev. E 70, 0419061-0419067 (2004)

84. W. Gutkowski and T. A. Kowalewski, Mechanics of the 21st century, Springer, Warsaw, Poland (2005).

85. K. M. Van Citters, B. D. Hoffman, G. Massiera and J. C. Crocker, "The role of F-actin and myosin in epithelial cell rheology," Biophys. J. 91,3946-3956 (2006) 
86. H. J. Butt, B. Cappella and M. Kappl, "Force measurements with the atomic force microscope: technique, interpretation and applications," Surf. Sci. Rep. 59, 1-152 (2005)

87. M. Radmacher, "Studying the mechanics of cellular processes by atomic force microscopy," Method Cell Biol. 83, 347-372 (2007)

88. H. W. Wu, T. Kuhn and V. T. Moy, "Mechanical properties of L929 cells measured by atomic force microscopy: Effects of anticytoskeletal drugs and membrane crosslinking," Scanning 20(5), 389-397 (1998)

89. J. Alcaraz, L. Buscemi, M. Grabulosa, X. Trepat and B. Fabry, "Microrheology of human lung epithelial cells measured by atomic force microscopy," Biophys. J. 84, 2071-2079 (2003)

90. B. A. Smith, B. Tolloczko, J. G. Martin and P. Gruetter, "Probing the viscoelasic behavior of cultured airway smooth muscle cells with atomic force microscopy: stiffening induced by contractile agonist," Biophys. J. 88, 2994-3007 (2005)

91. T. Okajima, M. Tanaka, S. Tsukiyama, T. Kadowaki, S. Yamamoto, M. Shimomura and H. Tokumoto, "Stress relaxation of HepG2 cells measured by atomic force microscopy," Nanotechnology 18, 084010084015 (2007)

92. T. Okajima, M. Tanaka, T. Tsukiyama, T. Kadowaki, S. Yamamoto, M. Shimomura and H. Tokumoto, "Stress relaxation measurement of fibroblast cells with atomic force microscopy," Jpn. J. Appl. Phys. 46(8B), 5552-5555 (2007)

93. S. L. Crick and F. C. P. Yin, "Assessing micromechanical properties of cells with atomic force microscopy: importance of the contact point," Biomechan. Model Mechanobiol. 6, 199-210 (2007)

94. E. A. Hassan, W. F. Heinz, M. D. Antonik, N. P. Dcosta, S. Nageswaran, C. A. Schoenenberger and J. H. Hoh, "Relative Microelastic mapping of living cells by atomic force microscopy," Biophys. J. 74, 1564-1578 (1998)

95. L. Scheffer, A. Bitler, E. Ben-Jacob and R. Korenstein, "Atomic force pulling: probing the local elasticity of the cell membrane.," Eur. Biophys. J. 30, 83-90 (2001)

96. T. Ohashi, Y. Ishii, Y. Ishikawa, T. Matsumoto and M. Sato, "Experimental and numerical analyses of local mechanical properties measured by atomic force microscopy for sheared endothelial cells," BioMed. Mater. Eng. 12, 319-327 (2002) 


\section{Chapter 2}

\section{Materials and Methods}

This chapter gives an overview of materials and methods used in this thesis work. First, the cell lines, fluorescent cell staining and cellular interventions using various drugs are introduced. Second, ballistic microinjection is discussed in detail, covering the principle, procedure and the quality assessment. The key experiments in this thesis are intracellular particle tracking (IPT) and combined atomic force microscopy and confocal scanning laser microscopy (AFM-CSLM) experiments. These techniques including calibrations will be discussed in the last part of this chapter. 


\subsection{Cells}

\subsubsection{Cell culture}

Human microvascular endothelial cells (Hmec-1, a gift from Dr. P. Koolwijk, TNO, Leiden, The Netherlands) at 25-30 passages, are cultured at $37{ }^{\circ} \mathrm{C}$ in a humidified $5 \% \mathrm{CO}_{2}$ environment in endothelial cell growth medium (EGM) containing hydrocortisone, hFGF, $\mathrm{R}^{3}$-IGF-1, ascorbic acid, hEGF, gentamicin, heparin, and $2 \%$ fetal bovine serum (EGM-2, Lonza, Basel, Switzerland). The Cell culture procedure is as follows: 1) cells are grown on a standard T25 (25 ml) culture flask till confluent; 2) cells are flushed with phosphate buffer saline (PBS) twice to replace the medium; 3) $1 \mathrm{ml} 0.1 \times$ EDTA-trypsin is added to trypsinize cells in a thermal incubator for 2-3 minutes, until majority of the cells have detached from the surface of the culture flask; 4) immediately hereafter, $3 \mathrm{ml}$ of EGM-2 medium is added to neutralize the trypsin; 5) the cell suspension is centrifuged in a tabletop centrifuge at $1200 \mathrm{rpm}$ for $5 \mathrm{~min}$, followed by resuspending the cell pellet in $1 \mathrm{ml}$ of EGM-2 medium by gently mixing; 6) 1/3 of the suspension is transferred to a T25 culture flask pre-coated with Fibronectin $(100 \mu \mathrm{g} / \mathrm{mL}$ solution for $1 \mathrm{~h}) ; 7)$ the cells are left in a thermal incubator at $37^{\circ} \mathrm{C}$, $5 \% \mathrm{CO}_{2}$ for growing.

Before IPT or AFM-CSLM experiments, $1 \mathrm{ml}$ of cell suspension is plated on a Delta $\mathrm{T}$ culture dish (Bioptechs, Butler, PA, USA), previously coated with fibronectin $(100 \mu \mathrm{g} / \mathrm{mL}$ solution). At least 3 hours in thermal incubator are given to allow cell attachment.

Besides Hmec-1, also various tumor cells are studied (a gift from Dr. J. Schnekenburger). Human pancreas adenocarcinoma Patu8988S (PA-S), and Patu8988T (PA-T) cells are cultured in Dulbecco's modified eagle medium containing 5\% FBS, 5\% horse serum and 1\% (2mM) L-glutamine (DSMZ, Germany). Human breast epithelial adeno-carcinoma (MCF-7) cells are cultured in RPMI 1640 (Lonza) containing 10\% FCS, 1\% (2mM) L-glutamine, $10 \mu \mathrm{g} / \mathrm{ml}$ insulin, $1 \mathrm{mM}$ sodium pyruvate and non-essential amino acids. Human breast fibrocystic epithelial cells (MCF-10A) are cultured in MEGM (Lonza).

The culture procedure is similar to that for endothelial cells. However, a difference is that the culture flask does not need pre-coating with fibronectin. In trypsinization $0.25 \times$ EDTA-trypsin is used to incubate with cells. For malignant cells, the trypsinization takes 2-3 min; for benign tumors, 5-10 min is needed.

For each tumor cell line, samples at 2-5 passages are studied. Cells to be analyzed with AFM are grown on a Delta-T culture disk until they reach 80 - 
$100 \%$ confluence, and are kept in $\mathrm{CO}_{2}$ independent medium under physiological conditions until the measurements are completed. This typically lasts $\sim 3-4$ hours.

\subsubsection{Immunofluorescent staining}

\subsubsection{1, Live cell imaging}

To image the cell boundary and nucleus in fluorescent imaging mode, DiI (Vybrant DiI cell staining solution, Invitrogen) is applied to stain cellular lipid structures (Fig.2.1(3)). The optimum protocol is a 1:100 dilution in serum-free medium for 15 minutes, then removing the medium containing DiI, and flushing with PBS 3 times. Application of EGM on the culture flask then renders the cells ready for imaging under standard conditions.

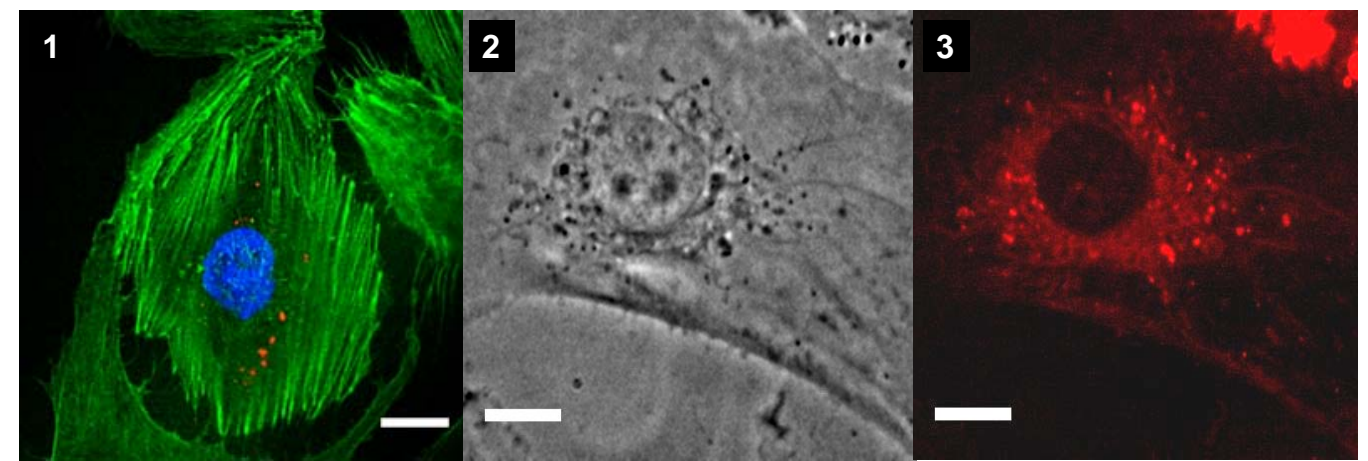

Fig. 2.1, (1), Multi-color fluorescence image showing actin fibers (labeled in green by FITC-phalloidin), the nucleus (labeled in blue by DAPI) and $200 \mathrm{~nm}$ ballistically injected particles (in red) in a HMEC-1 endothelial cell. (2), Phasecontrast image showing endogenous granules (appearing as black spherical spots) in an endothelial cell. (3), Nile-Red staining image of the same cell as in (2). Scale bar is $10 \mu \mathrm{m}$.

We also apply the lipophilic dye Nile Red for staining the endogenous granules, to confirm they are mainly neutral lipid droplets (Fig.2.1 (2)). The protocol is as follows: A Nile Red stock solution of $1 \mathrm{mg} / \mathrm{ml}$ in acetone is prepared; then diluted 10,000 times in serum-free medium to stain cells for $15 \mathrm{~min}$ in a thermal incubator, after which the liquid is replaced by normal medium.

\subsubsection{2, Fixed cell imaging}

To stain the intracellular F-actin network, cells are rinsed with PBS solution and fixed with $4 \%$ formaldehyde in PBS at $37{ }^{\circ} \mathrm{C}$ for $8 \mathrm{~min}$. Next, the cells are permeabilized with a PBS solution containing $0.2 \%$ Triton-X, $0.1 \%$ BSA (Sigma-Aldrich). FITC-phalloidin $(1 \mu \mathrm{M})$ is applied for $30 \mathrm{~min}$ to stain F-actin structures and DAPI (300 nM) is used for 5 min to stain cell nuclei (Fig.2.1 (1)). 
In order to stain microtubule network, samples are further immersed into a solution of $1 \mathrm{mg} / 1$ Rhodamine conjugated goat anti-mouse antibody for 20 minutes. Cell samples are rinsed with PBS afterwards. Immunofluorescence is visualized using an inverted confocal microscope (Zeiss, Germany) with $63 \times$ oil immersion objective.

\subsubsection{Drug and temperature interventions}

All drugs are purchased from Sigma Aldrich, except Blebbistatin (Tebu-bio, Belgium). Drugs are administered by adding them directly to the cell medium whilst the cells are on the microscope. Subsequent delivery to the cell interior then takes place via diffusion through the medium and transport across the cell membrane. The following drug interventions are done: Latrunculin A (200 nM, 2 h), Jasplakinolide (25 nM, 1h), Nocodazole (5 $\mathrm{MM}, 3 \mathrm{~h})$, Cephalomannine (taxol, $100 \mu \mathrm{M}, 1 \mathrm{~h})$, Blebbistatin ( $50 \mu \mathrm{M}, 2 \mathrm{~h})$. 2-D-deoxyglucose and sodium azide at final concentrations of $50 \mathrm{mM}$ and $0.05 \%$ are used for $30 \mathrm{~min}$ as an ATPdepletion cocktail. Each drug intervention effect is analyzed by 2-5 repetitive experiments.

The functions of these drugs are as following. Latrunculin A can bind to actin monomers to promote depolymerization of the actin network in both vivo and vitro [1,2]. Jasplakinolide has been reported to have two effects: 1) to stabilize the actin network in the cortex area [3] and 2) to destabilize the actin network in intracellular region [4]. Nocodazole can depolymerize the microtubule network by blocking the $\beta$-tubulin subunit [5]. Cephalomannine can 'freeze' microtubules by blocking their depolymerization [6] [7]. Blebbistatin [8] is a specific drug to interfere with actin and myosin contraction. Specifically noticed, blebbistatin has been reported [9] to create dose-dependent cell death at wavelengths of 365 and $450-490 \mathrm{~nm}$, but not in the ranges $510-560$ or $590-650 \mathrm{~nm}$. In our IPT experiments, a $\mathrm{Kr}$ laser line $(564 \mathrm{~nm})$ and a TRITC filter $(565 \mathrm{~nm} / 610 \mathrm{~nm})$ are used. ATP depletion is as described [10] to deplete intracellular ATP in the living cells.

Besides drug interventions, also the temperature is varied. This is done using the Bioptechs heating system. After reducing temperature from $37^{\circ} \mathrm{C}$ to $24^{\circ} \mathrm{C}$, a minimum of $30 \mathrm{~min}$ is given to reach thermal equilibrium.

\subsubsection{Temperature control and calibration for IPT and AFM experiments}

To keep the cells at $37{ }^{\circ} \mathrm{C}$ while they are studied on the microscope, they are cultured on a Delta-T dish (Bioptechs) which has a conductive and transparent ITO layer at the bottom. A controlled electrical current is sent through the ITO to 
supply heat. The temperature calibration of the stage is shown in Fig. 2.2. In particle tracking experiments also a heated lid and an objective heater (Bioptechs) are used. Due to water condenstation at the side-wall of the Delta-T dish, cell viability can be maintained for $\sim 6$ hours. Alternatively, a layer of light embryonic oil (Sigma) on top of the cell medium can prevent the problem of water condensation. However, in that case the cell medium can not be replaced anymore. For our IPT experiment, generally heating lid is used to keep the temperature equilibrium.

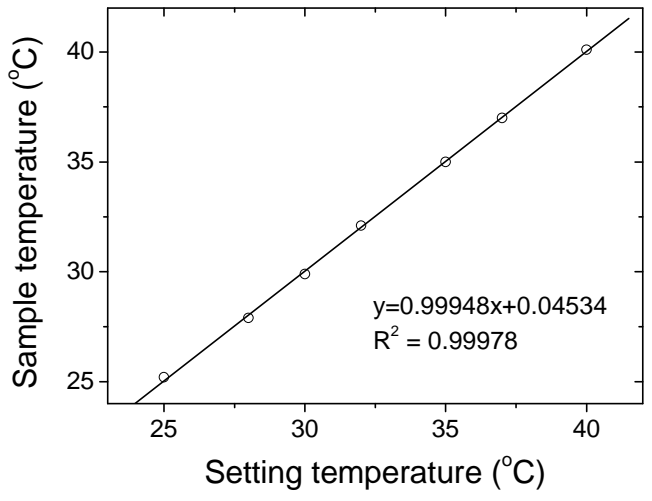

Fig. 2.2, Temperature calibration curve of Delta-T heating stage. Sample temperature is measured using a small Pt-100 sensor that is held in contact with the upper surface of the stage.

\subsection{Ballistic microinjection}

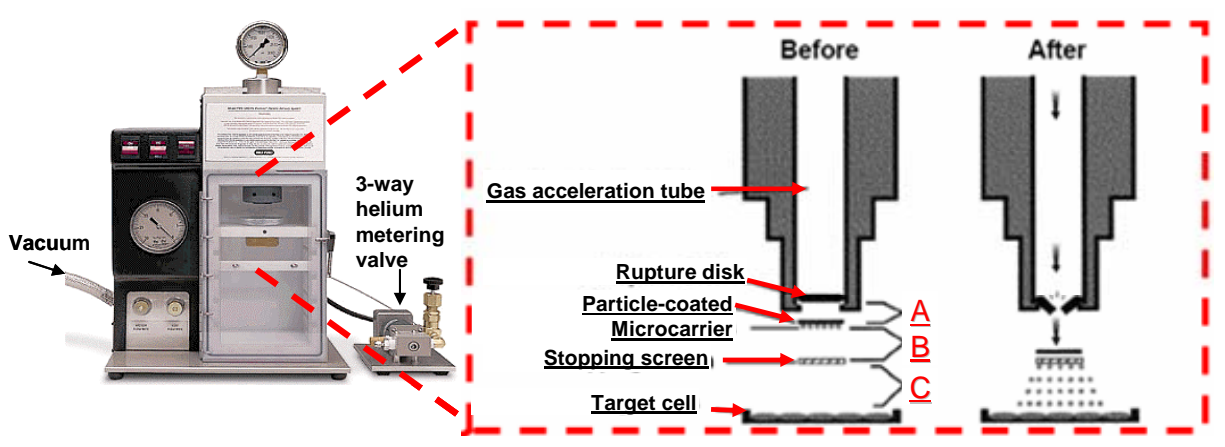

Fig.2.3, Biolistic PDS-1000/He particle delivery system [11].

Ballistic microinjection is a method that uses helium pressure to introduce many nanoparticles simultaneously into living cells. The working principle of the instrument (shown in Fig. 2.3) is to release a pulse of high pressure helium gas 
after the breaking of a rupture disk. This high pressure will then propel (at high velocity) a so-called microcarrier disk loaded with millions of microspheres towards target cells. The disk is stopped once it reaches the stopping screen, however, the nanoparticles which carry the momentum will continue to travel towards the cell culture plate and penetrate the cell membranes. This technique was originally developed for genetic transfection using typically metal particles with a size of $\approx 1 \mu \mathrm{m}$. Panorchan et al [12] extended this application to the injection of polystyrene latex particles with size 100-500 nm. For each particle/cell combination, the injection conditions have to be optimized. A too low particle speed will not lead to injection and a too high speed will destroy the cells. Key parameters are the helium pressure (related with the rupture disk), the vacuum level in the bombardment chamber, the distance from the rupture disk to the microcarrier (A in Fig.2.3), the distance between microcarrier disk and stopping screen (B), and the distance between the stopping screen and the target cells (C).

\subsection{1, Preparation of nanoparticles and microcarriers}

We modify the protocol of Panorchan [12] to prepare microcarrier disks with a dense coating of microspheres, while largely avoiding aggregation of the particles.

The nanoparticles used in our study are carboxylated fluorescent polystyrene spheres with diameter 200nm (Invitrogen) originally in a $2 \% \mathrm{w} / \mathrm{v}$ aqueous suspension. To ensure high quality coating, the nanoparticle suspension is firstly diluted in $100 \%$ ethanol and centrifuged at $8000 \mathrm{rpm}$ for 20 minutes in a Micromax RF microcentrifuge (IEC). The supernatant is then discarded and the particle pellet is resuspended in $100 \%$ ethanol. This procedure is repeated 3 times, resulting eventually in a $2 \% \mathrm{w} / \mathrm{v}$ suspension in ethanol. The particle suspension is further sonicated for 5 minutes to disperse the particles and disaggregate eventual clusters.

Preparation of the microcarrier disks is performed using a spincoater (Special Coating Systems, Inc.). Microcarriers are immersed in isopropanol prior to the coating for cleaning. The procedure is 1) to put a microcarrier disk on the spincoater set a rotation speed of $1000 \mathrm{rpm}$, and let it dry, 2) to pipette $20 \mu \mathrm{l}$ of particle suspension on the microcarrier and carefully spread the particles with the pipette from the center to the edge of the disk while spin-coating, 3) when the particle smears are dry, to pipette an additional $20 \mu \mathrm{l}$ and repeat the same procedure, 4) to leave the microcarrier to dry for at least $1 \mathrm{hr}$ at $50^{\circ} \mathrm{C}$ in an oven or overnight at room temperature. 


\subsection{2, Ballistic injection of nanoparticles}

Bombardment conditions are optimized by exploring different settings for the rupture pressure, vacuum, and the distances $\mathrm{A}, \mathrm{B}$ and $\mathrm{C}$ as shown in table 2.1.

Table 2.1, Optimization of ballistic bombardment conditions.

\begin{tabular}{|c|c|c|c|c|c|}
\hline $\begin{array}{l}\text { Helium } \\
\text { Pressure } \\
\text { (psi) }\end{array}$ & $\begin{array}{l}\text { Vaccum } \\
\text { (mmHg) }\end{array}$ & $\begin{array}{l}\text { Distance } \\
\text { (A) }\end{array}$ & $\begin{array}{l}\text { Distance } \\
\text { (B) }\end{array}$ & $\begin{array}{l}\text { Distance } \\
\text { (C) }\end{array}$ & Results \\
\hline 1100 & 28 & $1 / 4$ inch & $5 \mathrm{~mm}$ & $3 \mathrm{~cm}$ & $\begin{array}{l}50-60 \% \text { survival; } 5-10 \% \text { with } 1- \\
2 \text { particles; } 1 \% \text { 10-20 particles }\end{array}$ \\
\hline 1100 & 28 & $\begin{array}{l}1 / 8 \\
\text { inch }\end{array}$ & $15 \mathrm{~mm}$ & $3 \mathrm{~cm}$ & $\begin{array}{l}20-30 \% \text { survival; almost no } \\
\text { particles }\end{array}$ \\
\hline 1100 & 28 & $\begin{array}{l}1 / 8 \\
\text { inch }\end{array}$ & $15 \mathrm{~mm}$ & $6 \mathrm{~cm}$ & $\begin{array}{l}80-90 \% \text { survival; almost no } \\
\text { particles }\end{array}$ \\
\hline 1350 & 28 & $\begin{array}{l}1 / 8 \\
\text { inch }\end{array}$ & $5 \mathrm{~mm}$ & $3 \mathrm{~cm}$ & $\begin{array}{l}30-40 \% \text { survival; } 5-10 \% \text { with } \\
5-10 \text { particles }\end{array}$ \\
\hline 1350 & 28 & $1 / 4$ inch & $5 \mathrm{~mm}$ & $3 \mathrm{~cm}$ & $\begin{array}{l}40-50 \% \text { survival; most of cells } \\
\text { have particles; } 5 \%>20 \\
\text { particles }\end{array}$ \\
\hline 1350 & 28 & $1 / 4$ inch & $15 \mathrm{~mm}$ & $3 \mathrm{~cm}$ & $\begin{array}{l}20-30 \% \text { survival; } 10 \% 1-5 \\
\text { particles, a few }>10 \text { particles }\end{array}$ \\
\hline 1350 & 28 & $\begin{array}{l}1 / 8 \\
\text { inch }\end{array}$ & $15 \mathrm{~mm}$ & $6 \mathrm{~cm}$ & $\begin{array}{l}70-80 \% \text { survival; } 1 \%<10 \\
\text { particles }\end{array}$ \\
\hline 1550 & 28 & $1 / 4$ inch & $5 \mathrm{~mm}$ & $3 \mathrm{~cm}$ & $\begin{array}{l}20-30 \% \text { survival; most of cells } \\
\text { have } 1-2 \text { particles, few with } 5- \\
10 \text { particles }\end{array}$ \\
\hline 1550 & 28 & $1 / 4$ inch & $5 \mathrm{~mm}$ & $6 \mathrm{~cm}$ & $\begin{array}{l}80-90 \% \text { survival; few cells have } \\
\text { particles }\end{array}$ \\
\hline
\end{tabular}

For all our living mammalian cells and $200 \mathrm{~nm}$ polystyrene spheres, ballistic bombardments are performed using $1350 \mathrm{psi}(\sim 9.3 \mathrm{MPa})$ rupture disks, $28 \mathrm{~mm}$ $\mathrm{Hg}(\sim 3.7 \mathrm{kPa})$ vacuum, distance A (Fig. 2.3$)=1 / 4$ inch $(6.35 \mathrm{~mm})$, distance B $=6$ $\mathrm{mm}$ and distance $\mathrm{C}=30 \mathrm{~mm}$. The bombardment procedure is as follows: 1) load the rupture disk, particle coated microcarrier and stopping screen; 2) remove the supernatant (except for a thin layer) from the cell culture, put the cell culture dish in the middle of the sample compartment (and close the door); 3) allow the vacuum pressure to reach $28 \mathrm{~mm} \mathrm{Hg}$ and quickly switch to hold mode; 4) allow the helium gas to pass through the gas acceleration tube till the rupture disk bursts; 5) release the vacuum in the chamber; 6) take the sample and intensively flush it with fresh medium, to prevent endocytosis of non-injected particles. 
After a 30 min waiting time for cell recovery, cells are trypsinized. The cell suspension is centrifuged in a table top centrifuge at $1200 \mathrm{rpm}$ for 5 minutes; after which the supernatant containing the suspended (i.e. non-injected) particles is discarded. Then the cell pellet is resuspended and cells are plated in a new culture flask precoated with $100 \mu \mathrm{g} / \mathrm{mL}$ fibronectin; Cells are allowed to adhere on the new substrate for at least 3 hours before the particle tracking experiments. Cells that have died do not adhere and can hence easily be removed. Using optimized injection parameters, we achieve $\approx 50 \%$ survival rate of the cells, and the cells contain up to 15-40 particles per single Hmec-1 cell.

\section{3, Intracellular particle tracking (IPT)}

The setup used for the intracellular particle tracking experiments is shown in Fig. 2.4. To perform experiments under physiological conditions, a heating stage, a heated lid and an objective heater (Bioptechs) are used to keep cells at $37^{\circ} \mathrm{C}$ and $5 \% \mathrm{CO}_{2}$, which is supplied as a 5/95\% $\mathrm{CO}_{2}$ /air mixture at $\sim 0.2$ bar overpressure. Before supplying this gas to the cells, it is moisturized by feeding it through a closed chamber containing $5 \% \mathrm{CO}_{2}$ saturated water. No significant difference in particle dynamics is found with or without $\mathrm{CO}_{2}$ supply. Hmec-1 cells can survive $\sim 6 \mathrm{~h}$ without significant morphological changes.

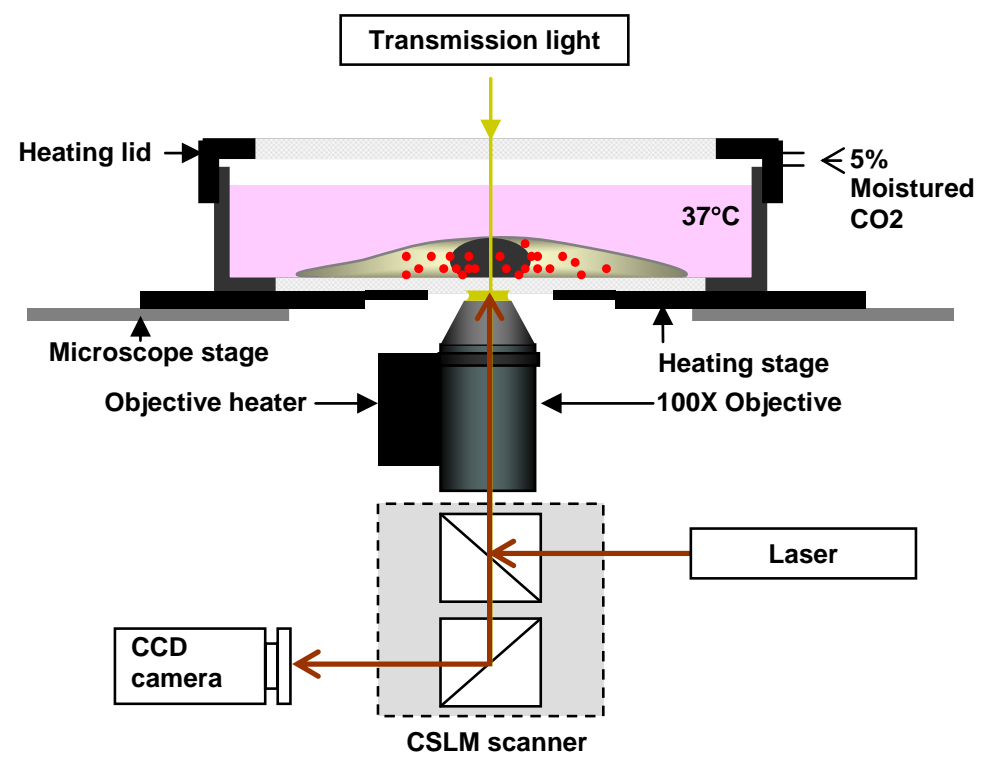

Fig.2.4, Schematic view of the setup used for intracellular particle tracking. 


\subsubsection{Phase Contrast and Confocal Scanning Laser Microscopy (CSLM)}

To visualize living cells, $100 \times$ phase contrast imaging module is implemented on a Nikon TE300 inverted microscope. As described elsewhere [13], phase contrast imaging is a conventional technique that employs an optical mechanism to translate minute variations in phase (due to different refractive index of the examined specimen in comparison to the surrounding medium) into corresponding changes in amplitude, which can be visualized as differences in image contrast. In phase contrast imaging, the separated surrounding and diffracted wavefronts emerging from the specimen are projected onto different locations in the objective rear focal plane (the diffraction plane at the objective rear aperture) causing an increased brightness of background light and a reduced brightness of diffracted light from objects with higher refractive index.

For our CSLM we use an UltraView LCI10 system (Perkin Elmer), containing a $20 \mathrm{~mW}$ Ar/Kr combined laser, a $300 \mathrm{~W}$ Xenon lamp, a Nikon Eclipse TE300 inverted microscope, a Yokogawa confocal unit based on the double Nipkow disk principle, and a 12-bit Hamamatsu CCD camera. A 100x oil objective with NA 1.3 is used for particle tracking. With this system, a pixel resolution of 0.13 $\mu \mathrm{m}$ per pixel, and a minimum exposure time of $60 \mathrm{~ms}$ per frame can be reached. CSLM is a valuable tool for obtaining high resolution images and 3-dimensional reconstruction of samples [13]. In our CSLM, a laser beam is passing through a rotating spinning disk with pinholes. This pinhole blocks all out-of focus light of both excitation and emission and allows sample illumination / detection at very small focal volume, thus increasing the resolution of imaging.

\subsubsection{Probe types}

Two kinds of intracellular probe particles are intensively studied in this thesis, ballistically injected particles (BIPs) and endogenous granules (EGs). The EGs are confirmed to be predominantly lipid droplets as proven by staining with Nile Red (Invitrogen) as shown in Fig 2.1 (3). These granules have a mean diameter of $\sim 0.5 \mu \mathrm{m}$. They appear as dark objects (Fig.2.1 (2)) in phase contrast imaging mode. For ballistic injection red-fluorescent carboxylated polystyrene particles with a diameter of $0.2 \mu \mathrm{m}$ are used (Invitrogen). They appear as bright red spots in confocal fluorescence imaging mode when using a $\mathrm{Kr}(561 \mathrm{~nm})$ laser line and a TRITC filter $(535 \mathrm{~nm} / 615 \mathrm{~nm})$. Image 2.1 (1) shows a typical Hmec-1 cell with injected fluorescent particles. 


\subsubsection{Multiple particle tracking}

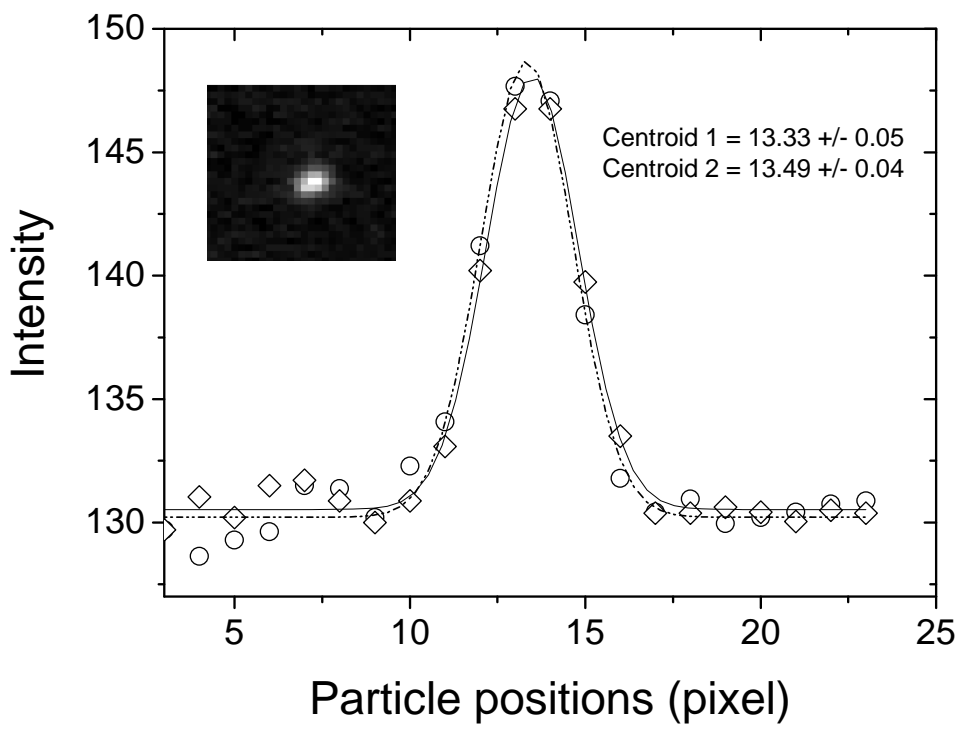

Fig. 2.5, Positions of a particle in two consecutive images. Inset shows the fluorescent image of the particle.

The particles are localized using the publicly available particle-tracking code based on the paper by Crocker and Grier [14], written and extended in Interactive Data Language (IDL, Creaso) by several authors. Excellent tutorials can be found online, e.g. [15]. Essentially, a brightness-weighted centroid algorithm is used to localize the particles in two dimensions. Then from the time dependence of the particle position, a trajectory is constructed. The image of a typical particle spans $\sim 5 \times 5$ pixels (Fig. 2.5 inset). The location of the centroid is determined by 2D Gaussian fitting of the intensity profile, with an accuracy of $\sim 0.05$ pixels, corresponding to $0.05 \times 0.13 \mu \mathrm{m}=6.5 \mathrm{~nm}$. Images of probe particles are taken at a constant rate. The particle position in two consecutive images can be determined as shown in Fig. 2.5. By analyzing the displacement of the centroid of a particle over a certain lag time $\tau$, one can compute the mean squared displacement (MSD) in $2 \mathrm{D}$,

$$
\left\langle\Delta r^{2}(\tau)\right\rangle=\left\langle[x(t+\tau)-x(t)]^{2}+[y(t+\tau)-y(t)]^{2}\right\rangle,
$$

where $<>$ means time average.

Several parameter settings are critical to define a 'valid particle' for IPT study. A detailed description can for example be found at [15]. Briefly, spatial filtering is applied to reject image features that were too small (e.g. pixel noise) or too large 
(e.g. aggregates) to correspond to individual particles. Particles that are almost out of the focal plane are rejected via their intensity and area. Then finally, to construct particle trajectories, a maximum displacement criterion have to be used to facilitate finding the same particle unambiguously in subsequent images.

For some probe/cell combinations, particle displacements can get rather small $(<50 \mathrm{~nm})$ To assess the accuracy of localization, an experiment was conducted by bombarding particles directly on a glass surface and by measuring the MSDs of these immobilized particles with the same experimental conditions as for the cell experiments. This resolution is assessed to be $10-15 \mathrm{~nm}$ (Fig. 2.6).

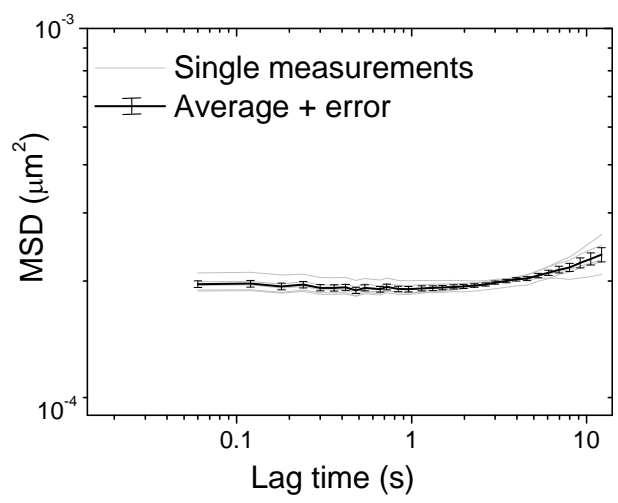

Fig.2.6, Mean square displacements of particles immobilized on a glass surface.

\subsection{Combined AFM-CSLM experiments}

The basic principle of AFM was discussed in Chapter 1. We use a home-built AFM head [16] interfaced to a commercial Veeco Nanoscope III controller (Fig. 2.7). This AFM scanner is placed on top of our CSLM microscope, to allow selection of the cells and alignment of the AFM tip with respect to the cell (Fig. 2.8). Confocal Scanning Laser Microscopy is a conventional tool that allows high resolution imaging and 3D scanning of a variety of samples, including living cells. To facilitate visualization, the cells were stained by DiI (Invitrogen) and to increase cell viability for AFM measurements, they are incubated in $\mathrm{CO}_{2}$ independent medium (to allow optimum $\mathrm{pH}$ value). A Piezo stage (Physik Instrumente) controls the $\mathrm{X}, \mathrm{Y}$ and $\mathrm{Z}$ positions (range: $200 \times 200 \times 200 \mu \mathrm{m}$ ) of the sample stage to allow the positioning and alignment. 


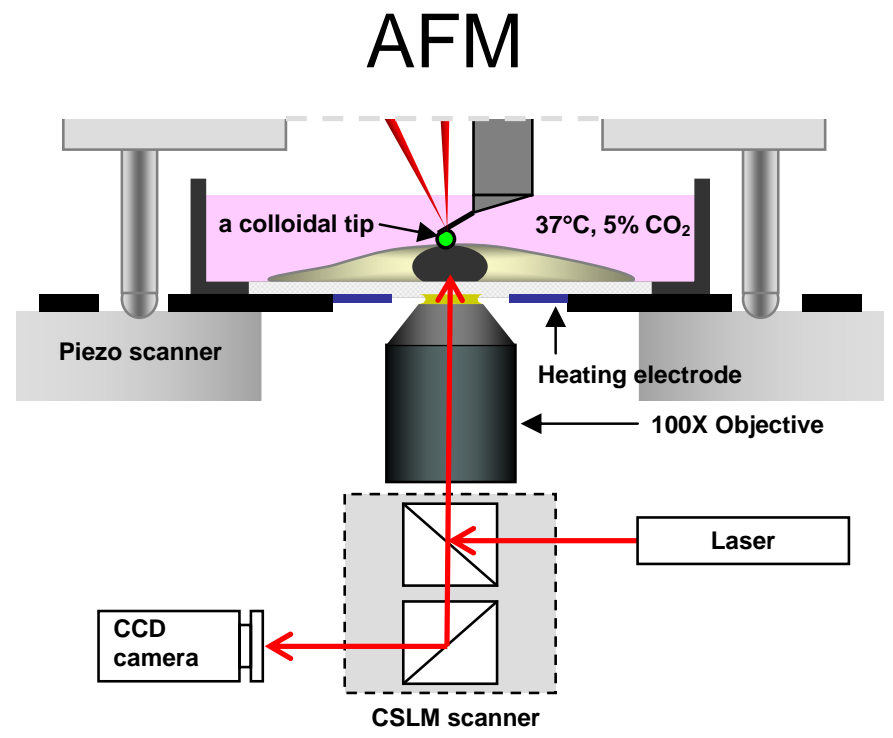

Fig. 2.7, Schematic view of a cell indentation experiment using AFM-CSLM.

An impression of an AFM indentation experiment visualized with the CSLM can be seen in Fig. 2.8.

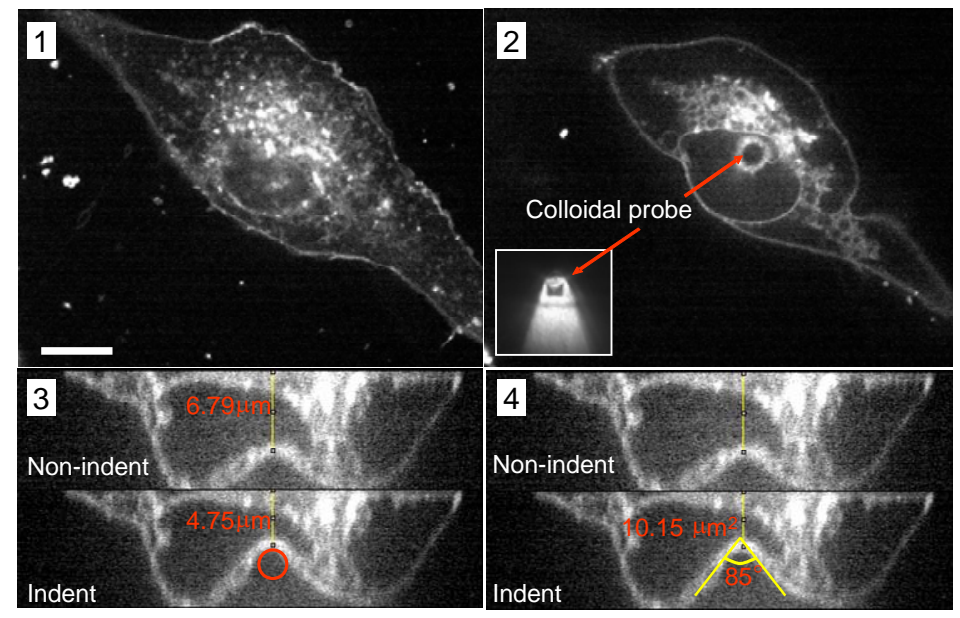

Fig. 2.8, Confocal images of a Hmec-1 cell indented with a $3.7 \mu$ m diameter glass sphere (1) a Hmec-1 cell stained by DiI. Scale bar: $10 \mu \mathrm{m}$ (2) the cell is indented by a colloidal probe (see arrow). Inset shows a colloidal tip in reflective imaging mode. (3) Figures indicate distance of probe to substrate. Indentation depth can be calculated as $1.78 \mu \mathrm{m}$ (4) Figures show contact angle and contact area of probe on cell ( $\triangle A)$. Pressure at maximum indentation can be calculated as $F / \Delta A$. 


\subsubsection{Force distance experiments}

Cell elasticity measurements are done in force-distance mode using silicon nitride $\left(\mathrm{Si}_{3} \mathrm{~N}_{4}\right)$ cantilevers (Microlevers) having an experimentally determined spring constant of $0.03 \mathrm{~N} / \mathrm{m}$ and a pyramidal tip with a half opening angle of $15^{\circ}$. Typical approach-retraction and force-distance curves are shown in Fig. 2.9. The tip is positioned on top of the center of the nucleus.

To find the contact point between the tip and the cell, the AFM tip is gently lowered via the piezo-stage control unit, until a significant deflection increase is found. At that point the tip is retracted to be out-of-contact. After setting the ramping frequency and amplitude, force-distance curves are collected for 3-5 times at the center of the nuclear location on the cell. To verify that the indentation itself does not cause immediate changes in the cell's mechanical response, 10-20 subsequent measurements are collected at the same spot (on top of the nuclear region). It is then ascertained that the values of $E^{*}$ do not show significant differences.

A more accurate determination of the contact point is obtained by a least-squares fitting of a quadratic dependence to the force-indentation depth curve with an accuracy of $\sim 50 \mathrm{~nm}$. In experiments aimed at revealing the dependence of $\mathrm{E}^{*}$ on loading rate, cells are indented at loading amplitude $2.45 \mu \mathrm{m}$ and different loading frequencies ranging from $0.05-2 \mathrm{~Hz}$, which is equivalent to speeds of $246 \mathrm{~nm} / \mathrm{s}-9.82 \mu \mathrm{m} / \mathrm{s}$.

A typical AFM experiment lasts about 4-5 hours including 30 min of thermal stabilization after the tip is introduced into the liquid phase. To obtain an optimum signal to noise ratio, cells are indented typically at $2-8 \mathrm{nN}$, which corresponds with indentation depth of about $1 \mu \mathrm{m}$. 

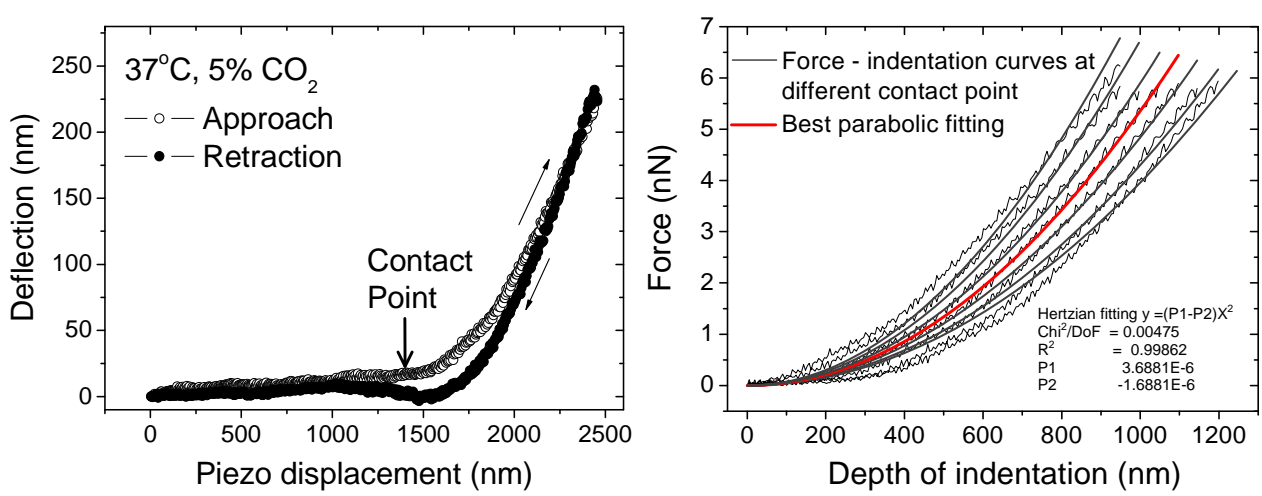

Fig. 2.9, Left, approach-retraction curves of a PA-T cell indented at loading speed $2.45 \mu \mathrm{m} / \mathrm{s}$. Right, force-indentation curves at different contact points (see approach-retraction curve) varying from $1500 \mathrm{~nm}$ to $1200 \mathrm{~nm}$ (left to right). The red line indicates the best fitting which gives apparent Young's modulus $\sim 1 \mathrm{kPa}$.

Table 2.2, Comparison of Hertzian fitting parameters of the force-indentation curves in Fig. 2.9 at different contact points.

\begin{tabular}{|c|r|r|r|r|r|r|r|}
\hline Contact point $(\mathrm{nm})$ & $1200 \mathrm{~nm}$ & $1250 \mathrm{~nm}$ & $1300 \mathrm{~nm}$ & $\mathbf{1 3 5 0} \mathbf{n m}$ & $1400 \mathrm{~nm}$ & $1450 \mathrm{~nm}$ & $1500 \mathrm{~nm}$ \\
\hline Chi^2 $^{\wedge}$ & 0.0475 & 0.0349 & 0.0122 & $\mathbf{0 . 0 0 5 3}$ & 0.0065 & 0.0266 & 0.0566 \\
\hline $\mathrm{R}^{\wedge} 2$ & 0.9877 & 0.9909 & 0.9968 & $\mathbf{0 . 9 9 8 6}$ & 0.9983 & 0.9929 & 0.9844 \\
\hline $\mathrm{E}^{\star}(\mathrm{Pa})$ & 0.7981 & 0.8437 & 0.9161 & $\mathbf{0 . 9 8 5 3}$ & 1.0588 & 1.1706 & 1.2772 \\
\hline
\end{tabular}

To ensure the accuracy of fitting, we do a comparison of the parabolic fitting for the force-indentation curves at different contact points (Fig. 2.9). The fitting parameters are listed in table 2, in which the Bold numbers indicate the best fit. For different contact point varying $+/-150 \mathrm{~nm}$ from the best fitting point (1350 $\mathrm{nm})$, about $20-25 \%$ inaccuracy of elastic moduli $(\sim 1 \mathrm{kPa})$ can be assumed. This value is consistent with the literature reported value [17]. In our experiments, accuracy of $+/-50 \mathrm{~nm}$ can be achieved, which is equivalent to $6 \sim 7 \%$ inaccuracy.

\section{References:}

[1] Spector, I., Shochet, N.R., Kashman, Y., and Groweiss, A., 1983, Latrunculins: novel marine toxins that disrupt microfilament organization in cultured cells, Science, 219 (4584), pp. 493-495.

[2] Coué, M., Brenner, S. L., Spector, I., and Korn, E. D., 1987, Inhibition of actin polymerization by latrunculin A. FEBS Lett. 213, pp. 316-318. 
[3] Bubb, M.R., Senderowicz, A.M.J., Duncan, K.L.K., and Korn, E.D., 1994, Jasplakinolide, a cytotoxic natural product, induces actin polymerization and competitively inhibits the binding of phalloidin to F-actin, J. Biol. Chem., 269, pp. 14869-14871.

[4] Michael, R.B., Spector, I., Beyer, B.B., and Fosen, K.M., 2000, Effects of jasplakinolide on the kinetics of actin polymerization, J. Biol. Chem., 275, pp. 5163-5170.

[5] Vasquez et. al, 1997, Nanomolar concentrations of nocodazole alter microtubule dynamic instability in vivo and in vitro, Mol.Biol.Cell, 8, pp. 973.

[6] Schiff, P.B., and Horwitz, S.B., 1980, Taxol stabilizes microtubules in mouse fibroblast cells, Proc. Natl. Acad. Sci., 77, pp. 1561-1565.

[7] Yvon, A.C., Wadsworth, P., and Jordan, M.A., 1999, Taxol Suppresses dynamics of individual microtubules in living human tumor cells, Mol. Biol. Cell, 10, pp. 947-959.

[8] Kovacs, M., Toth, J., Hetenyi, C., Csizmadia, A.M., and Sellers, J.R., 2004, Mechanism of blebbistatin inhibition of myosin II, J. Biol. Chem., 279, pp. 35557-35563.

[9] Kolega, J., 2004, Phototoxicity and photoinactivation of blebbistatin in UV and visible light, Biochem. Biophys. Res.Commu., 320, pp.1020-1025.

[10] Hoffman, B.D., Massiera, G., Van Citters, K.M., and Crocker, J.C., 2006, The consensus mechanics of cultured mammalian cells, Proc. Natl. Acad. Sci. U. S. A., 103, pp.10259-10264.

[11] Manual of Biolistic PDS-1000/He particle delivery system, Bio-Rad, Califonia, 2000.

[12] Panorchan, P., Lee, J. S., Daniels, B. R., Kole, T. P., Tseng, Y., and Wirtz, D., 2006, Probing cellular mechanical responses to stimuli using ballistic intracellular nanorheology, Meth. Cell. Biol., 83, pp. 115-140.

[13] Douglas, M., 2001, Fundamentals of Light Microscopy and Electronic Imaging, John Wiley \& Sons., USA.

[14] Crocker, J.C., Grier, D.G., 1996, Methods of digital video microscopy for colloidal studies, J.Colloid Int. Sci. 179, 298-310.

[15] http://www.physics.emory.edu/ weeks/idl/index.html.

[16] Van der Werf, K.O., Putman, C.A.J., Degrooth, B.G., Segerink, F.B., Schipper, E.H., Van Hulst, H.F., Greve, J., 1993, Compact stand-alone atomic-force microscope, Review of Scientific Instruments, 64(10),pp. 2892-2897.

[17] Crick, S. L., Yin, F. C-P, 2007, Assessing micromechanical properties of cells with atomic force microscopy: importance of the contact point, Biomechan. Model Mechanobiol., 6, pp. 199-210 


\title{
Chapter 3
}

\section{Mapping of Spatiotemporal Heterogeneous Particle Dynamics in Living Cells}

\begin{abstract}
Colloidal particles embedded in the cytoplasm of living mammalian cells have been found to display remarkable heterogeneity in their amplitude of motion. However, consensus on the significance and origin of this phenomenon is still lacking. We conducted experiments on Hmec-1 cells loaded with about 100 particles, to reveal the intracellular particle dynamics as a function of both location and time. Central quantity in our analysis is the amplitude (A) of the individual Mean Squared Displacement (iMSD), averaged over a short time. Histograms of $A$ were measured: 1) over all particles present at the same time and 2) for individual particles as a function of time. Both distributions showed significant broadening compared to particles in Newtonian liquid, indicating that the particle dynamics varies with both location and time. However, no systematic dependence of $A$ on intracellular location was found. Both the (strong) spatial and (weak) temporal variations were further analyzed by correlation functions of $A$. Spatial cross- correlations were rather weak down to interparticle distances of 1 micron, suggesting that the precise intracellular probe distribution is not crucial for observing a dynamic behavior that is representative for the whole cell. Temporal correlations of $A$ decayed at $\sim 10 \mathrm{~s}$, possibly suggesting an intracellular reorganization at this time scale. These findings imply 1) that both individual particle dynamics and the ensemble averaged behavior in a given cell can be measured if there are enough particles per cell, and 2) that the amplitude and power-law exponent of iMSDs can be used to reveal local dynamics. We illustrate this by showing how super- and subdiffusive behaviors may be hidden under an apparently diffusive global dynamics.
\end{abstract}




\subsection{Introduction}

Recent literature has witnessed a strong increase in the use of colloidal particles as local tracers of dynamic processes in soft materials [1,2]. What makes these particles so suitable is that they are both small enough to show Brownian motion, and large enough to probe the deformations of the soft material network. Then the motion of each particle is driven by mechanical excitations from surrounding molecules on one hand, and damped by its viscoelastic microenvironment on the other. Conversely, information about these forces can also be obtained by analyzing the statistics of particle motion. A good example is the study of passive engineering materials with particle tracking microrheology (PTM); here the assumptions that all particle motions are driven by thermal collisions and that the fluctuation-dissipation theorem (FDT) applies, are used to calculate viscoelastic properties of the material from the Mean Squared Displacement (MSD) of the particles as a function of lag time [3].

Alternatively, also the interior (i.e. cytoplasm) of living biological cells has been studied with the methods from PTM [4-20]. Measuring the statistical motions of (endogenous or microinjected) particles provides a direct way to study intracellular mechanics at (sub) micron lengthscales. Yet there are also certain challenges involved. In the earliest PTM studies on soft biological matter it was recognized that besides the size, also the chemistry of the probe can have strong influence on the MSD functions obtained [9, 21, 22]. More recently, also contributions of ATP dependent processes to particle dynamics have been identified [17, 19, 23]. Together, these studies have resulted in a general consensus that the FDT is violated, and that intracellular particle MSDs can reflect thermal or ATP dependent driving forces, viscoelastic damping, or combinations thereof. which information can be obtained from MSDs measured inside living cells then depends on the experimental conditions and on the time and/or lengthscales addressed. Restricting the analysis to short lag times [23], and suppressing active motion by ATP depletion [13] are possibly strategies to measure viscoelastic properties of the cytoplasm. Alternatively, in cases where probes cannot be assumed to be chemically inert and driven by thermal collisions, their MSDs can still provide unique information. For example, probes attached to specific intracellular structures (like the actin network or microtubules), can reveal structural or dynamic changes in these networks.

An additional aspect encountered in intracellular studies is that the dynamic behavior varies from particle to particle. Considering the complexity and structural heterogeneity of living cells, this finding is not surprising, but it also 
raises the question of how to deal with this heterogeneous dynamics. Most studies have been aimed at measuring a behavior representative for the whole cell. For single large $(4 \mu \mathrm{m})$ beads [13] this was achieved by temporal averaging, while in studies with several small $(100-500 \mathrm{~nm})$ particles distributed over the cell $[6,7,10,14]$, both spatial and temporal averaging were used to obtain an overall MSD. However, that obtaining such a representative MSD from just a few particles per cell is far from trivial is also clear, considering that the MSD amplitude sometimes varies over more than an order of magnitude $[4,5,9,15,16]$.

Relatively few studies have been aimed at studying these heterogeneities themselves. Using multiple particle tracking (one-point microrheology, 1PMR), Heidemann and Wirtz [4] and Kole et al [5] reported cytoplasmic stiffening near the leading edges of migrating Swiss 3T3 fibroblasts, in agreement with the required local functionality of the cell. In another study, nonmigrating fibroblasts were reported to have a mechanical compliance that depended on the distance from the nucleus. This was described as an intrinsic mechanical heterogeneity [9]. Alternatively, other researchers addressed the aspect of spatial heterogeneity via both one- and two-point microrheology (2PMR) [15,16,24]. Since the $\mathrm{D}_{\mathrm{rr}}$ correlator was found to scale with interprobe distance $r$ as $\sim 1 / r$ for $2 \mu \mathrm{m}<r<8$ $\mu \mathrm{m}$ (as expected for a homogeneous medium), Van Citters et. al. concluded that gross-scale mechanical heterogeneity was absent in TC7 epithelial cells. However experiments with a single large bead per cell had shown large amplitude variations from cell to cell $[15,16]$. These were attributed to variations in (adhesive) contact between probe and matrix. In a study by Bursac et. al., a relation between binding state and MSD behavior was found for externally attached beads [19]. Thus in literature, different physical origins have been suggested for the spatially heterogeneous dynamics found in cells: mechanical heterogeneity $[4,5]$ and a variability in tracer-cytoskeleton contacts $[16,19]$.

The temporal aspect of heterogeneity has been addressed even less, even though the potential role hereof has been pointed out $[15,20,25,26]$. A clear example is the intermittent dynamics [20], displayed by particles that are for short episodes actively transported via linkage to a motor protein, but otherwise free to diffuse. Also less conspicuous temporal variations can occur. For example, cytoskeleton remodeling can expose a particle to a new microenvironment, or a change in the binding state between probe and matrix can cause a change in dynamics. The consequences of such events on time averaged MSDs have hardly been explored so far. 
Thus a lot is still missing in our understanding of the spatiotemporal heterogeneity of intracellular particle dynamics. This lack of knowledge can seriously obstruct interpretation of intracellular particle tracking experiments, in several respects. First it raises the question, under which conditions a 'blind averaging' over intracellular MSDs will produce a total MSD that is representative for the whole cell. Second, due to temporal fluctuations, also the analysis of the individual MSDs may become obscured. By default one might assume that during the timespan in which particles are tracked, the dynamic behavior does not change. But if for example a particle switches intermittently between two simple dynamic behaviors, and the integration time over the trajectory is too long, then these individual behaviors will be washed out, producing an iMSD that is hard to interpret. And third, a lack of knowledge on the phenomenology of spatiotemporal heterogeneity also obstructs finding its physical origin(s).

In this paper we present a detailed study of the spatial and temporal heterogeneity in the dynamics of intracellular particles. Two goals were pursued. First, we wanted to assess the relative importance of spatial and temporal heterogeneity in a chosen type of cell, and measure the characteristic length and time scales over which the dynamic behavior of individual particles can change. And second, we wanted to explore tools to characterize mechanically heterogeneous materials in general. Besides biological cells also many engineering materials display spatial or spatiotemporal heterogeneities. Examples are associating polymer solutions [27], gels [25,28,29], protein suspensions [30], gelled and jammed colloids [1,31], and two phase materials $[32]$.

For the present work we chose to analyze the dynamics of endogenous granules (EGs) in living Hmec-1 cells (also artificially introduced latex particles called BIPs will be considered, albeit in less detail). This intracellular probe has been studied before $[10,13,14,33,34]$, and is known to display heterogeneous dynamics when studied with 1PMR [10]. We will use the iMSD amplitude measured at the shortest lag time as the central quantity. Its dependence on location and time will be studied via correlation functions and variance analysis. To achieve sufficient accuracy we analyze a large data set containing $\approx 10^{5}$ trajectories, of which a significant fraction has long duration (>1000 time steps). Significance of the measured heterogeneities will be assessed by comparing the results to reference cases: experiments in viscous liquid and computer simulations for particles showing Brownian motion. 
Importantly, we will conclude that our particles have distinguishable dynamics within the $150 \mathrm{~s}$ timescale of our experiments. Building on this outcome, we then examine the distributions in MSD amplitude and power-law exponent. We will show that both quantities show a significant distribution, for both EGs and BIPs. The broad range of power-law exponents indicates that not only the motion amplitude, but also the qualitative dynamic behavior of the same kind of particles present inside the same cell, can be very different.

This chapter is further organized as follows. In section 3.2 we describe the technical details of the experiments and the computer simulations. In section 3.3 we develop a number of statistical tools to analyze MSDs and illustrate some of their properties using numerically simulated MSD traces. In section 3.4 we will present a quantitative analysis of the spatial and temporal heterogeneity displayed by EGs in confluent Hmec-1 cells. Based on these findings, we present in Section 3.5 an extended analysis, covering also the different types of dynamic behavior of EGs and BIPs in individual Hmec-1 cells. Conclusions will be drawn in Section 3.6.

\subsection{Experiments}

\subsubsection{Cell Culture}

Human microvascular endothelial cells (Hmec-1, a gift from Dr. P. Koolwijk, TNO, Leiden, The Netherlands) at 25-30 passages, were cultured at $37{ }^{\circ} \mathrm{C}$ in a humidified $5 \% \mathrm{CO} 2$ environment in endothelial cell growth medium containing hydrocortisone, hFGF, R3-IGF-1, ascorbic acid, hEGF, gentamicin, heparin, and $2 \%$ fetal bovine serum (EGM-2, Lonza, Basel, Switzerland). Cells were plated on a Delta $\mathrm{T}$ culture dish (Bioptechs, Butler, PA, USA), precoated with fibronectin $(100 \mu \mathrm{g} / \mathrm{mL}$ solution) and mounted on an inverted microscope before experiments. The dish bottom contains a thin ITO layer, whose temperature was controlled via a heating system (Bioptechs). A heated lid was used to seal the culture dish, and 5\% CO2 was supplied continuously.

\subsubsection{Probe Types}

We used two intracellular probes - endogenous granules (EGs) and ballistically injected particles (BIPs). The EGs, having a mean size of $\approx 0.5 \mu \mathrm{m}$, were confirmed to be mainly lipid droplets and some mitochondria by staining with Nile Red and Rhodamine dyes respectively. These granules appear as dark objects under phase contrast microscopy. For the BIPs we chose fluorescently labeled carboxylated polystyrene spheres with diameter of $0.2 \mu \mathrm{m}$ (Invitrogen). These particles were introduced via ballistic injection as described by Panorchan et. al. [6] and visualized by illuminating with an $\operatorname{Ar}$ laser $(\lambda=488 \mathrm{~nm})$. 


\subsubsection{Intracellular particle tracking}

Probe particles were visualized using a Nikon Eclipse TE300 inverted microscope coupled to a Confocal module (UltraView LCI 10, Perkin Elmer, Cambridge, UK). EGs and BIPs were imaged under phase contrast and fluorescence mode respectively using a $100 \mathrm{X}$ (NA 1.3) objective. Images were recorded with a 12-bit CCD camera (Hamamatsu IEEE 1394 C4742-95-12 ERG). The unit exposure time was set to $60 \mathrm{~ms}$ and the spatial resolution corresponding to the images was $0.13 \mu \mathrm{m}$ per pixel. Probe motion was studied in individual cells (both under confluent and nonconfluent conditions) that contained a large number of particles: typically 40 for BIPs and 80 for EGs. To obtain enough observations for our statistical analysis, we recorded in between 10 and 41 movies (of 2500 frames each) per cell. Statistical analyses (of correlations and variances, see Sec. 3) were always performed on a single cell. The time dependent locations of the particles were obtained using the available code [35], originally based on the paper of Crocker and Grier [36] and written and extended in Interactive Data Language (IDL). The error in our measurement of the particle displacements was $10 \mathrm{~nm}$.

\subsubsection{Computer simulations}

Brownian Dynamics simulations were performed to generate reference data for use in interpretation. The case of uniformly sized particles in a Newtonian liquid was mimicked by spreading typically 200 particles over an XY area, and subsequently letting each particle make an individual random 2D step for each time unit, for a total of 10000 steps per particle. $\mathrm{X}$ and $\mathrm{Y}$ displacements per particle and step were obtained by sampling from Gaussian distributions generated via the Box-Muller method as available in the IDL library. Particle trajectories thus generated were transformed into iMSDs as explained in Section 3.3 .

\subsection{Particle Tracking}

\subsubsection{Mean squared displacement functions}

To describe the dynamics of a single particle $p$, use can be made of its (individual) Mean Squared Displacement function:

$$
\Delta r_{p}^{2}(\tau)=\sum_{t=1}^{N_{t}(p, \tau)}\left[\left(x_{p}(t+\tau)-x_{p}(t)\right)^{2}+\left(y_{p}(t+\tau)-y_{p}(t)\right)^{2}\right] / N_{t}(p, \tau)
$$

with $x_{p}(t)$ and $y_{p}(t)$ representing the location of the particle at time $t$, and $\tau$ the lag time. Since the localizations are made from video images, both $t$ and $\tau$ are expressed in units of the exposure time per image: $t_{l}$. Then the number of contributions $N_{t}(p, \tau)$ is given by $N_{t}(p, \tau)=\left(T_{p}-\tau\right) / t_{1}$ with $T_{p}$ the duration of 
the trajectory of particle $p$. Averaging the iMSD over all particles then produces the total MSD,

$$
\Delta r^{2}(\tau)=\sum_{p=1}^{N_{p}} N_{t}(p, \tau) \Delta r_{p}^{2}(\tau) / \sum_{p=1}^{N_{p}} N_{t}(p, \tau)
$$

with $N_{p}$ the number of particles. This MSD is commonly used in particle tracking studies. Inspired by the dynamic behaviors displayed in some reference cases (diffusive motion in either elastic (a) or viscous (b) media, or ballistic motion (c)), measured MSD functions are often fitted with a power law function:

$$
\Delta r^{2}(\tau) \cong A\left(\frac{\tau}{\tau_{r e f}}\right)^{\alpha}
$$

with $A$ the amplitude at reference time $\tau_{\text {ref }}$ and $\alpha$ the power-law exponent (respectively 0,1 and 2 for the mentioned cases a, b and c). For particles embedded in linear viscoelastic materials, fractional exponents can be found, while also transitions in dynamic behavior can manifest themselves at a certain lag time. To account for such cases, use is made of local measures for $A$ and $\alpha$ :

$$
A=\Delta r^{2}\left(\tau_{r e f}\right), \alpha=\left[\frac{d \ln \left(\Delta r^{2}\right)}{d \ln \tau}\right]_{\tau_{r e f}}
$$

In principle, this description can also be applied to the individual MSD functions. An illustration can be found in Fig. 3.1, for a simulated set of particles in a Newtonian liquid: each of the iMSDs has an amplitude and power-law exponent close to that of the average. This demonstrates that even a single particle trajectory can already provide a (semi-) quantitative measure of the dynamics in the system. It is also seen that the iMSDs display a larger 'noise' in $A$ and $\alpha$ at longer lag times. This is a purely statistical effect, due to the random nature of the individual displacements, combined with the decreasing number of contributions $N_{t}(p, \tau)$ from which the averages are calculated. Fig. 3.1 thus shows both the utility of the iMSD and the potential limitations on its use. 


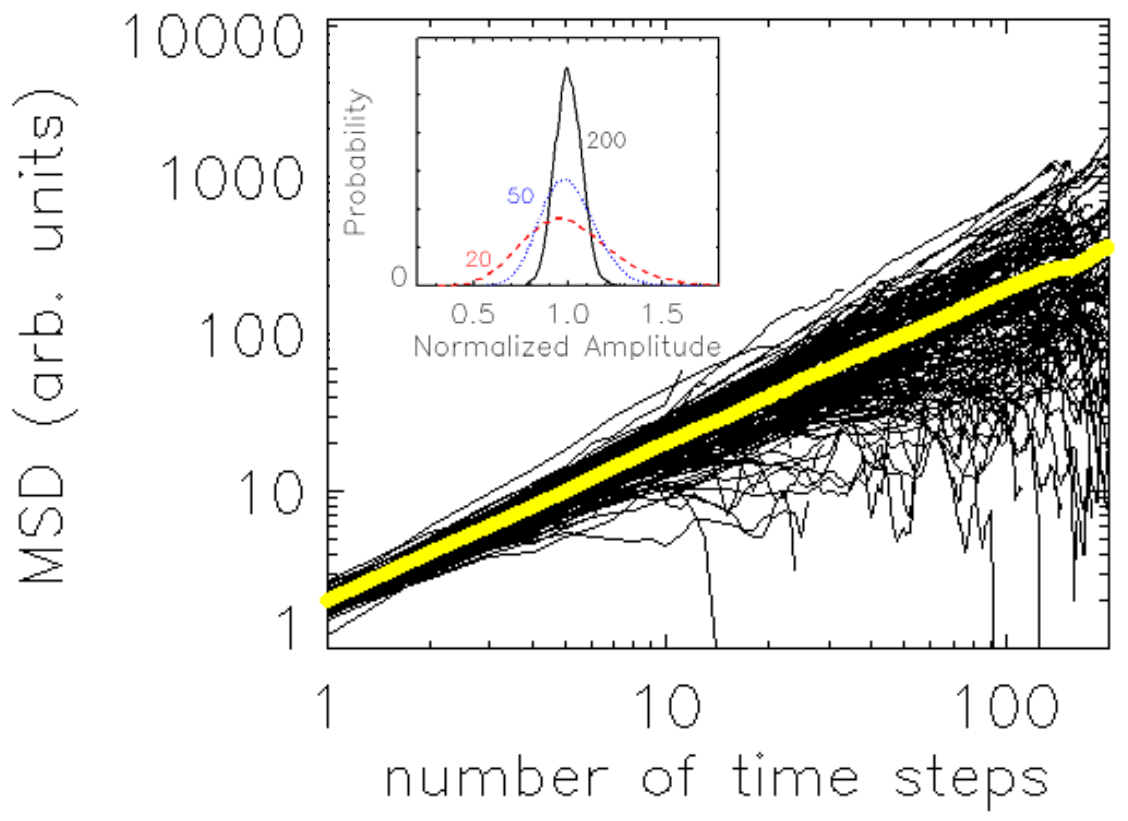

Fig.3.1: Individual Mean Squared Displacement functions obtained by simulation for a purely diffusive system. The yellow central line indicates the total MSD obtained by averaging over all particles. Inset: Normalized distribution of the iMSD amplitude, as a function of the number of contributions to the average: 20 (red dashes) 50 (blue dots) and 200 (black solid line).

\subsubsection{Segmentation}

In the analysis of iMSD functions, the relation between tracklength and noise level may need to be taken into account, especially when making statistical comparisons. One approach is to consider only (fragments of) trajectories that contain the same number of time steps. This number $(N)$ should be taken small enough to keep the fraction of lost (i.e. too short) trajectories low, and large enough to keep the noise level acceptable. The iMSD of such a fragment is then defined as:

$$
\left[\Delta r_{p}^{2}\right]_{N}(\tau)=\sum_{t=1}^{N-\frac{\tau}{t_{1}}}\left[\left(x_{p}(t+\tau)-x_{p}(t)\right)^{2}+\left(y_{p}(t+\tau)-y_{p}(t)\right)^{2}\right] /\left(N-\frac{\tau}{t_{l}}\right)
$$

which has $N-1$ contributions at $\tau=t_{1}, N-2$ at $\tau=2 t_{l}$, etc. In the remainder of this paper, our analysis will mainly be focused on the amplitude $\left(A_{p}\right)$ of this iMSD for $N=50$ and $\tau=t_{1}$ : 


$$
A_{p} \equiv\left[\Delta r_{p}^{2}\right]_{50}\left(t_{1}\right)
$$

\subsubsection{Spatial and time dependence}

The segmentation of trajectories into successive blocks of duration $50 * t_{1}$ also allows considering $A_{p}$ as a function of time:

$$
A_{p}\left(t^{\prime}\right)=\left\{A_{p}\left(t_{1}^{\prime}\right), A_{p}\left(t_{2}^{\prime}\right), A_{p}\left(t^{\prime}{ }_{3}\right), \ldots\right\}
$$

with

$$
t_{i}^{\prime}=50 *\left(i-\frac{1}{2}\right) * t_{1}+t^{\prime}{ }_{0}
$$

a new (coarse) time grid. Here $t^{\prime}{ }_{0}$ represents the real time at which the trajectory of particle $p$ started. By adding this time, the $A_{p}\left(t^{\prime}\right)$ functions are again synchronized for the different particles, so that all of them can be mapped onto a unique real-time grid $t^{\prime}$ (from now on designated as $t$ for notational convenience). In Fig. 3.2, an illustration is given of how $A_{p}(t)$ could look for two hypothetical particles (mimicked by computer simulation). The lower black curve represents a Brownian particle dispersed in a homogeneous liquid with constant viscosity; here the temporal variation of $A$ is purely due to statistical fluctuations. In contrast, the upper red curve corresponds to a particle which transfers to an environment with a higher viscosity. Now a decreasing trend is superimposed onto the fluctuating signal.

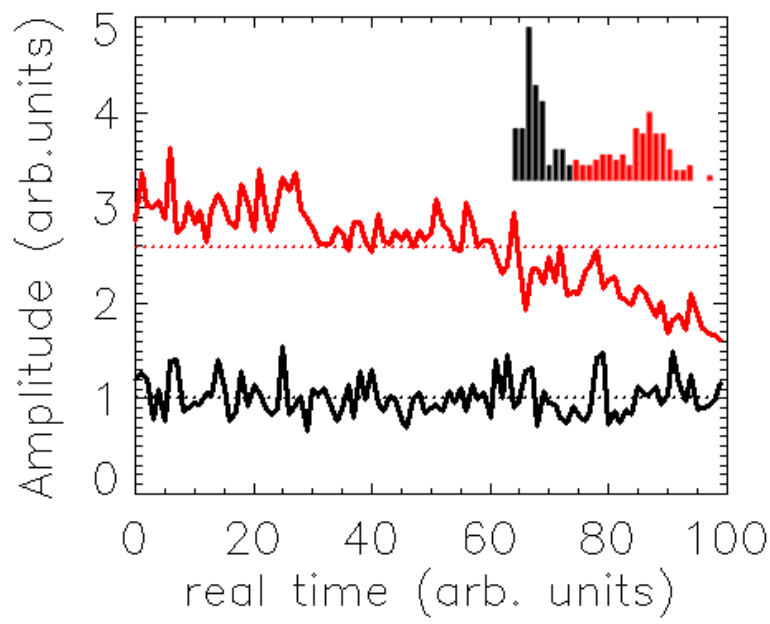

Fig.3.2: Illustration of two exemplary behaviors of the iMSD amplitude $A_{p}$ of particle $p$ as a function of real time. Dotted lines show the average over the 100 observations. The inset shows the corresponding histograms of $A_{p}$ values. 
Besides a real time, also a location can be assigned to $A$, as evident from the fact that each particle $p$ is localized (as a function of time) by the tracking procedure. Considering all particles $p$ present at the same time $t_{i}$, the position dependence of $A_{t_{i}}$ at that time is sampled at the locations $\left(x_{p}, y_{p}\right)$ :

$$
A_{t_{i}}(\underline{r})=\left\{A_{p 1}\left(x_{p 1}, y_{p 1}\right), A_{p 2}\left(x_{p 2}, y_{p 2}\right), \ldots\right\}
$$

Following this approach, the collection of all particle trajectories can be cast into a two dimensional matrix, of $A$-values as a function of particle index (columns) and real-time index (rows). Analysis within a row then allows to compare $A$ values for the same particle at different times (notation: $A_{p}(t)$ ), while analysis within a column allows to compare $A$-values for different particles observed at the same time (notation: $A_{t}(p)$ ). This is also illustrated in Fig. 3.3.

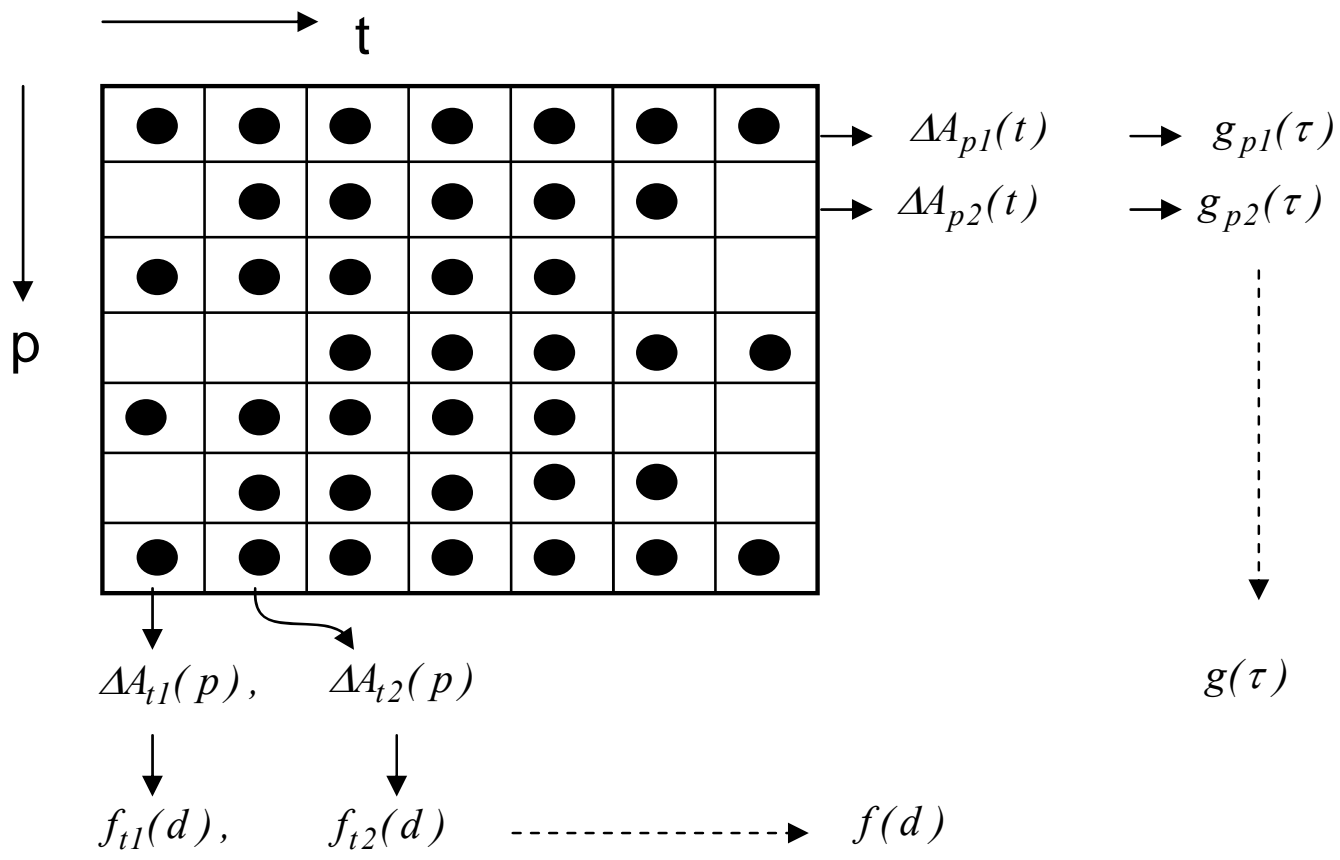

Fig.3.3: Schematic illustration of our analysis of iMSD amplitudes. P labels individual particles (and their positions) while $t$ is the index of a time segment in a chronological series. Filled circles indicate for which matrix elements $(p, t)$ a contribution to A exists. Analysis of the rows allows studying fluctuations of $A$ over time, via autocorrelation functions $g(\tau)$ or via variances. Analysis of the columns allows quantifying spatial variations of $A$ via cross correlations $f(d)$ or via variances.

\subsubsection{Time auto correlation function}


Temporal variations in the iMSD amplitude of a given particle can occur if the particle is transferred into a mechanically different environment. One way to study such changes in $A_{p}$ over time, is to calculate its time autocorrelation function. Defining first the temporal fluctuation $\Delta A_{p}(t)$ of an individual particle $p$ as:

$$
\Delta A_{p}(t) \equiv A_{p}(t)-<A_{p}>_{t}
$$

with $\left\langle A_{p}\right\rangle_{t}$ the time average:

$$
<A_{p}>_{t}=\sum_{t=1}^{N_{t}(p)} A_{p}(t) / N_{t}(p)
$$

and $N_{t}(p)$ the number of contributions (i.e. the number of elements in row $p$ where $A$ exists), the autocorrelation function of $A_{p}(t)$ is given by:

$$
g_{p}(\tau)=\frac{1}{N_{t}(p, \tau)} \sum_{t=1}^{N_{t}(p, \tau)} \Delta A_{p}(t) \Delta A_{p}(t+\tau) / \frac{1}{N_{t}(p)} \sum_{t=1}^{N_{t}(p)}\left[\Delta A_{p}(t)\right]^{2}
$$

with $N_{t}(p, \tau)=\left(T_{p}-\tau\right) / \tau_{1}$ i.e. as before but now with $\tau$ in units of $\tau_{1}=50^{*} t_{1}$. Note that $g_{p}(\tau)$ is normalized per particle. Obtaining a time autocorrelation function with an acceptable noise level requires a very large number of contributions. If this criterion is not met for the individual functions $g_{p}(\tau)$ then it may still be possible to obtain an autocorrelation function with an acceptable signal-to-noise ratio $(S / N)$, by averaging over all particles:

$$
g(\tau)=\sum_{p=1}^{N_{p}} N_{t}(p, \tau) g_{p}(\tau) / \sum_{p=1}^{N_{p}} N_{t}(p, \tau)
$$

This total autocorrelation function $g(\tau)$ will be analyzed in Sec. 3.4.

\subsubsection{Spatial cross correlation function}

Spatial variations of $A$ can be expected in materials where the mechanical properties and/or driving forces depend on location. Then having many probe particles spread out over the material, allows sampling the spatial distribution of these properties. To account for possible temporal variations (i.e. $A$ not only depending on location but also on time), spatial distributions of $A$ will only be considered for particles present at the same time. One way to analyze these is to calculate a spatial correlation function. For this we first define the local deviation $\triangle A_{t}$ (associated with particle $p$ ) as:

$$
\Delta A_{t}(p)=A_{t}(p)-<A_{t}>_{p}
$$

with $\left\langle A_{t}\right\rangle_{p}$ the ensemble average:

$$
<A_{t}>_{p}=\sum_{p=1}^{N_{p}(t)} A_{t}(p) / N_{p}(t)
$$


and $N_{p}(t)$ the number of contributions (i.e. the number of elements in column $t$ where $A$ exists). Then using the coupling between a particle's index $p$ and its position $\underline{r}$, the distance $d_{p q}$ between the centers of two particles $p$ and $q$ is calculated and subsequently binned onto an array $d_{i}=i * d_{1}$, with $d_{1}$ a chosen unit distance. Defining $C_{t, d_{i}}$ as the collection of all ${ }^{N} C_{t, d_{i}}$ particle pairs $(p, q)$ for which $d_{i} \leq d_{p q}<d_{i+1}$ at time $t$, we then calculate the intermediate function:

$$
X_{t}\left(d_{i}\right)=\sum_{p, q \in C_{t, d_{i}}}^{{ }^{N} C_{t, d_{i}}} \Delta A_{t}(p) \Delta A_{t}(q) / N_{C_{t, d_{i}}}
$$

which is then used to calculate the (normalized) spatial correlation function at time $t$ :

$$
f_{t}\left(d_{i}\right)=X_{t}\left(d_{i}\right) / X_{t}\left(d_{i}=0\right)
$$

Finally, averaging over all time segments t and generalizing for all $d_{i}$ then gives the total spatial (auto and cross) correlation function:

$$
f(d)=\sum_{t=1}^{T / \tau} 1 N_{C_{t, d_{i}}} f_{t}(d) / N_{C_{t, d_{i}}}
$$

This function is suited for revealing the presence or absence of a spatial correlation length for A, and will be analyzed in Sec. 3.4.

\subsubsection{Time Variance}

While correlation functions can provide detailed information about characteristic time or length scales at which a quantity (like $A$ ) shows a change, they also require a huge number of observations to achieve a good $S / N$ ratio. In cases where less data are available, one can still use (normalized) variances to quantify heterogeneity in the distribution of $A$. Using the definition Eq. (3.10), the variance in $A_{p}$ of a particle $p$ over time is given by:

$$
\operatorname{var}^{\text {time }}\left(A_{p}\right)=\frac{1}{N_{t}(p)-1} \sum_{t=1}^{N_{t}(p)}\left[\Delta A_{p}(t)\right]^{2}
$$

and transformed into a relative standard deviation with:

$$
\sigma_{\text {rel }}^{\text {time }}\left(A_{p}\right)=\sqrt{\operatorname{var}^{\text {time }}\left(A_{p}\right)} /<A_{p}>_{t}
$$

The average relative standard deviation corresponding to the entire particle set (i.e. all rows in Fig. 3.3) can then be formulated as:

$$
\sigma_{\text {rel }}^{\text {time }}=\sum_{p=1}^{N_{p}}\left(N_{t}(p)-1\right) \sigma_{\text {rel }}^{\text {time }}\left(A_{p}\right) / \sum_{p=1}^{N_{p}}\left(N_{t}(p)-1\right)
$$

It should be noted that the quantity expressed by Eq. 3.19 (and hence also Eqs. 3.20 and 3.21) may show an increase with the amount of time over which $A$ is 
observed: the longer this time, the more opportunity the particle is given to explore all its accessible values of $A$. For example if the analysis in Fig. 3.2 would have been restricted to 50 units rather than 100, then the broadest histogram would have been less broad. This aspect will be further considered in Sec. 3.4.

\subsubsection{Spatial Variance}

The spatial variance of the amplitude can be calculated in an analogous way as in Sec. 3.6, but now by considering columns instead of rows (Fig. 3.3). Then the spatial variance and relative standard deviation are defined for each column (i.e. time $t$ ) as:

$$
\operatorname{var}^{\text {spatial }}\left(A_{t}\right)=\frac{1}{N_{p}(t)-1} \sum_{p=1}^{N p(t)}\left[\Delta A_{t}(p)\right]^{2}
$$

and

$$
\sigma_{\text {rel }}^{\text {spatial }}\left(A_{t}\right)=\sqrt{\operatorname{var}^{\text {spatial }}\left(A_{t}\right)} /<A_{t}>_{p}
$$

The total average over all times is then calculated as:

$$
\sigma_{\text {rel }}^{\text {spatial }}=\sum_{t=1}^{N_{t}}\left(N_{p}(t)-1\right) \sigma_{\text {rel }}^{\text {spatial }}\left(A_{t}\right) / \sum_{t=1}^{N_{t}}\left(N_{p}(t)-1\right)
$$

\subsection{Results}

Fig. 3.4a shows a typical microscope image of a Hmec-1 cell loaded with endogenous granules (EGs). Tracking these particles over 50 steps of $60 \mathrm{~ms}$, calculating the iMSDs, and representing the amplitudes $A$ hereof (c.f. Eq. 3.6) with a color scale, results in Fig.3. 4b. This map (the graphical equivalent of Eq. 3.9) is representative for a set of 2000 of such images. It is shown that the particles are more or less evenly distributed over the accessible part of the cell interior (which excludes the nucleus and the actin cortex). Importantly, the amplitude $A$ varies appreciably between the particles, up to a factor $\approx 16$. This can also be seen from Fig. 3.4c, which shows representative iMSD functions (c.f. Eq. 3.1). The significance of this heterogeneity, compared to a Brownian particle system (analyzed in the same way), is apparent from the probability distributions in Fig. 3.4d.

Fig. 3.4b does not show any obvious systematic trends: neither particles close to each other nor particles close to the nucleus seem to display clearly visible correlations. The latter is in contrast with earlier findings [9] for carboxylated latex particles in Swiss $3 \mathrm{~T} 3$ fibroblasts. For our EGs in quiescent Hmec-1 cells 
there is clearly strong dynamic heterogeneity, but it does not seem linked to any large scale organization within the cell.
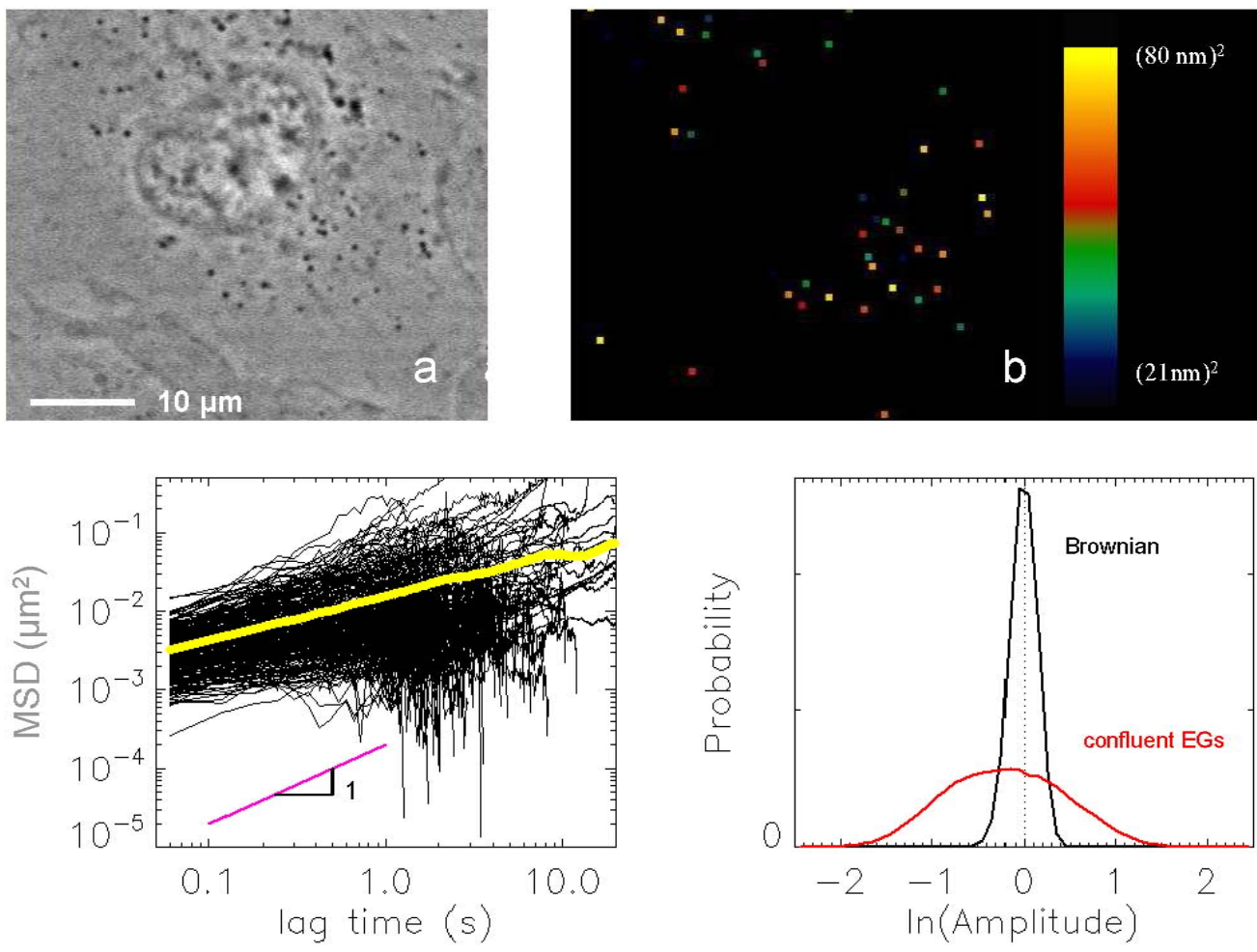

Fig.3.4: a) Microscopy image of a Hmec-1 cell containing endogenous granules, visible as dark objects. b) Reconstruction of a) in which each particle has been assigned an MSD amplitude, represented via a color scale. c) Individual and whole cell MSDs as found in a typical experiment. d) Amplitude histogram for EGs, averaged over all experiments. For comparison, also a histogram for Brownian particles is included. Note the amplitude normalization on the abscissa.

Further (and more quantitative) analyses were performed on the datasets underlying the amplitude maps. Here each dataset contained the real time index and for each particle its $(x, y)$ location and its iMSD amplitude $A$. Although our movies were taken consecutively, time correlations were only calculated within the same movie.

For our correlation analyses, we had to combine the data from all 41 movies to obtain an acceptable accuracy. For the spatial correlations, the number of particle pairs per image is important. On average there were 79 particles per image, giving $\approx 3000$ pairs. For the temporal correlations, both the number of tracks and 
their duration are important. Fig. 3.5 shows the histogram corresponding to the total dataset (before segmentation). Importantly, the number of trajectories longer than 50 steps is high $\left(>10^{4}\right)$ and a significant number of very long trajectories is obtained.

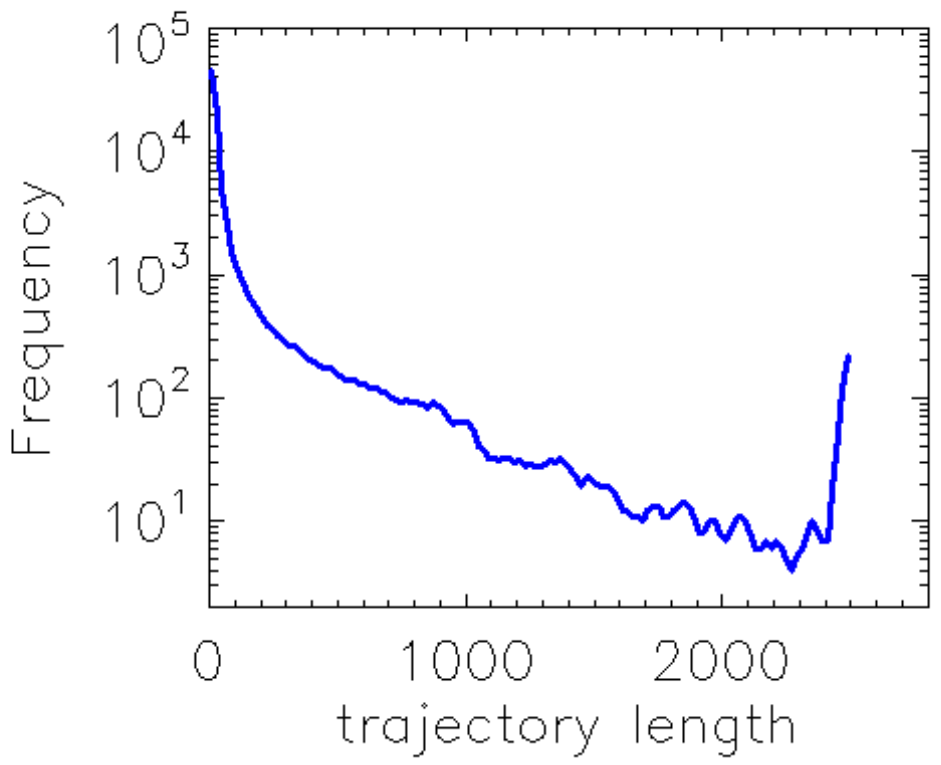

Fig.3.5: Histogram of trajectory lengths, obtained from 41 movies of Endogenous Granules in a confluent Hmec-1 cell.

The spatial correlation function for $A$ (c.f. Eq. 3.18) is shown in Fig. 3.6. The error bars display standard deviations calculated from the spread between the $f(d)$ functions calculated from the 41 individual movies. For comparison we also included the result of the same analysis procedure but now for carboxylated PS latex particles in glycerol (see inset). Probably due to the larger number of particles per image, the noise level is somewhat lower in the latter experiment. The correspondence with the zero level is very good, in line with expectations: for particles showing purely random (i.e. Brownian) motion, correlations between the motion amplitudes of different particles should indeed be absent. 


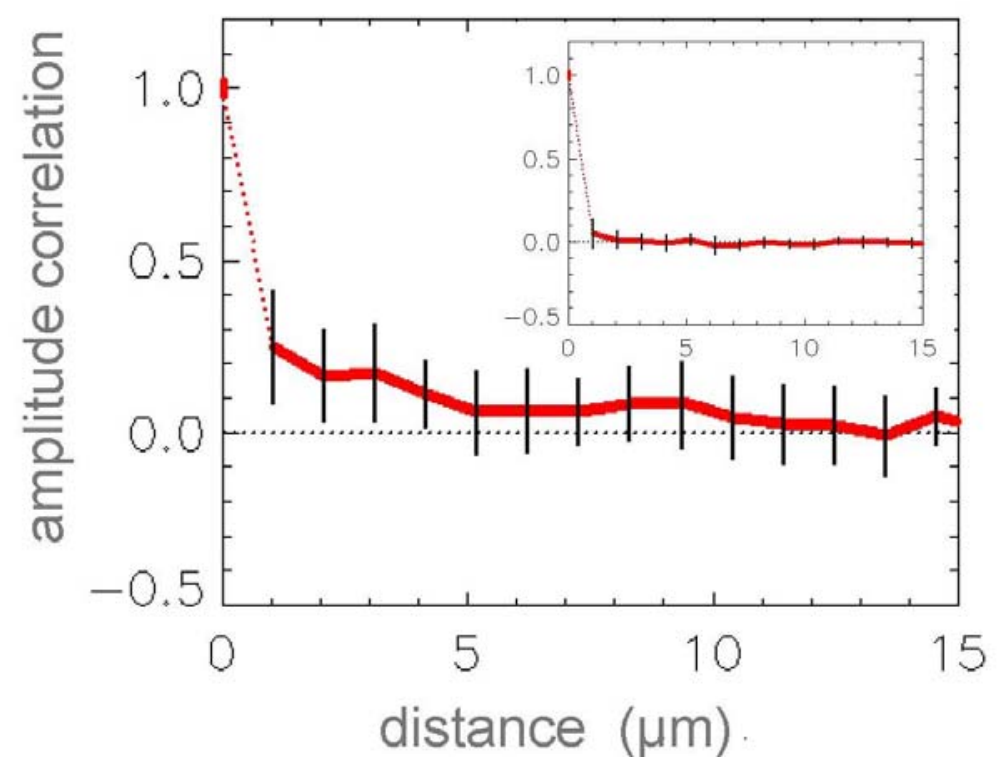

Fig.3.6: Spatial correlation function (c.f. Eq. 3.18) for the iMSD amplitude of EGs in Hmec-1 cells. Inset: reference case of Polystyrene latex particles in glycerol. Vertical bars indicate standard deviations.

For EGs in Hmec-1 cells, the magnitude of the correlation function lies mostly between 0 and 0.1 . Considering that the function is normalized to 1.0 , this suggests that correlations are either weak or absent. The slight upturn of the curve for distances below 4 microns might however still be significant. Making a more definitive statement, would require more particle pairs in close proximity. In our case interparticle separations of $1 \mu \mathrm{m}$ were already relatively sparse $\left(2 \cdot 10^{4}\right.$ contributions), and achieving a sufficient number of contributions at even smaller distances would require a prohibitively large number of movies. Probably for the same reason, Van Citters et. al. [16] were not able to calculate a reliable $\mathrm{D}_{\text {rr }}$ for distances smaller than $2 \mu \mathrm{m}$. As a last remark on this issue, we note that a fundamental lower limit on the spatial resolution would ultimately be set by the fact that the particles are mobile. In our case, the typical displacement over 50 time steps of $60 \mathrm{~ms}$ amounted to $\approx 40 \mathrm{~nm}$.

In summary, the results in Fig. 3.6 mainly confirm our expectation (based on inspection of many images like in Fig. 3.4b), that clear spatial correlations in iMSD amplitude are absent. This result corresponds well to the findings for TC7 epithelial cells, where a similar conclusion was drawn based on an analysis using two-point microrheology [16]. 
We now turn to time correlations. Fig. 3.7 shows the iMSD-time autocorrelation function (diamonds) as calculated from Eq. 3.13, averaging over trajectories from all available movies. For comparison, we also calculated the same function based on the same 41 movies, but now with trajectories segmented into blocks of 20 steps (squares). For both these calculations on EGs in Hmec-1 cells, we used only trajectories of 1000 or more unit time steps, so that at least 20 (respectively 50) time points were available for calculating the autocorrelation function. That this was an adequate criterion to ensure significant results is illustrated by the correspondence between the two curves.

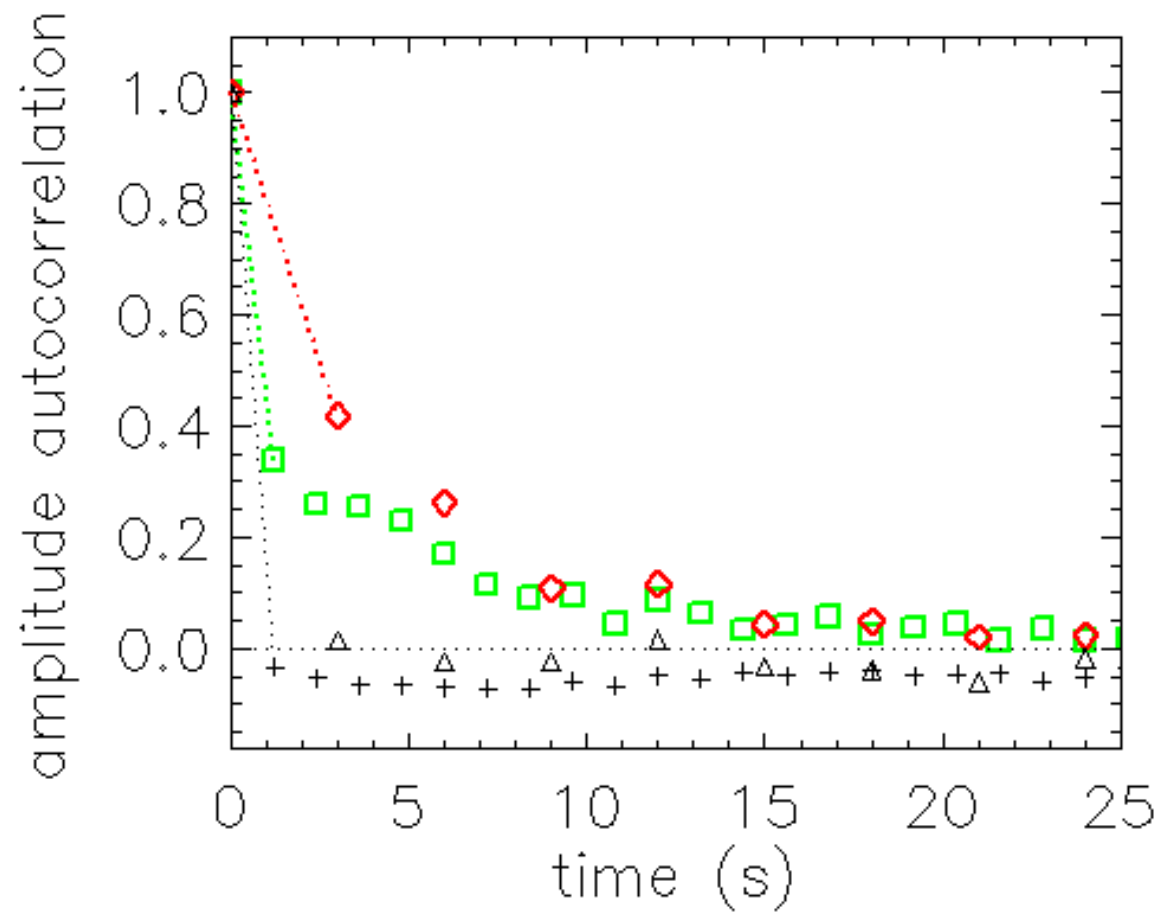

Fig.3.7: Time autocorrelation function (c.f. Eq. 3.13) of the iMSD of EGs in Hmec-1 cells. Red diamonds and green squares correspond to segmentation of trajectories into blocks of 50 and 20 units respectively of $60 \mathrm{~ms} .+$ : latex spheres in glycerol. 4: computer simulation for Brownian spheres.

Moreover we also applied this procedure to particle tracking experiments with latex particles in glycerol, and to data sets generated via Brownian Dynamics simulations. In the latter two cases zero correlation is expected for all lag times (except $\tau=0$ ), since in purely viscous systems the Langevin equation does not contain any memory term that links the current motion of a particle to its previous displacements. The reference data in Fig. 3.7 confirm the immediate 
loss of correlation, and the achievement of values very close to zero. The small negative deviation from zero displayed by the latex/glycerol data is attributed to the finite number of contributions to the correlation function.

Returning to the case of EGs in Hmec-1 cells, it is first of all clear that for times $>10 \mathrm{~s}$ no correlations can be detected. The upturn of the curves for correlation times shorter than $\approx 10 \mathrm{~s}$ suggests that the iMSD amplitude of endogenous granules in Hmec-1 cells takes about $10 \mathrm{~s}$ to decorrelate. Clearly this significant difference from the Brownian reference case suggests the presence of some kind of intracellular reorganization at this timescale. Whether this would be cytoskeleton remodeling, (un)binding to cytoskeletal elements, or simply cage rattling, cannot be stated and requires additional independent measurements.

We further note that while the plotted autocorrelation function indicates the existence of a correlation time, it does not reveal the magnitude of the changes in amplitude variation. To illustrate this: multiplying the deviation $\Delta A_{p}(t)$ given by Eq. 3.10 with an arbitrary constant will not change the correlation function given by Eq. 3.12. So while short-time temporal correlations are present, their quantitative contribution to the MSD amplitude heterogeneity still has to be assessed. Finally we remark that insufficient long trajectories were available to obtain a reliable correlation function for $\tau>25 \mathrm{~s}$. However the fact that $\mathrm{g}(\tau)$ already reaches near-zero values at $\tau=25 \mathrm{~s}$, implies that the time needed for a change in average $A$ comparable to the amplitude of the short-time fluctuations $\triangle A$, is $>25 \mathrm{~s}$.

To assess the relative importance of spatial and temporal variations in iMSD amplitude, we now turn to variance analysis. Analyzing distributions for variance does not require the vast amount of data as needed for the correlation functions, since only one number has to be calculated (rather than a binned distribution). To illustrate the idea we again consider Fig. 3.2, with the two hypothetical $A(t)$ profiles. Both profiles show fluctuations, but these are smaller than the difference between the average magnitudes of $A$. This is also shown by the bar histograms shown in the inset. Besides the average, also the variance is different for the two distributions. This is because upper profile not only fluctuates but also gradually decreases over time.

The comparison in Fig. 3.2 stands model for an important case: the difference in average iMSD between particles is larger than the variations in iMSD shown by individual particles in the experimental time-window (150 s). If this duration were very long, then both particles might explore the same set of accessible iMSD values (assuming the particles remain indistinguishable by nature) and the 
then resulting amplitude histograms would coincide. However on short timescales each particle explores only part of its 'iMSD configuration space'. Hence differences in the two variances: 1) over particles (i.e. space) and 2) over time, can reveal the presence of different micro-environments, as far as they are significant over the (150 s) timescale of the experiment.

Let us now consider the iMSD amplitude variations for EGs in Hmec-1 cells displayed in Fig. 3.8. Clearly, for each of the 41 recorded movies, the temporal variations within the trajectories are significantly smaller than the variations between particles. Moreover the relative standard deviation of the temporal variations is only slightly larger than the expected value of $0.20(\sqrt{2 / N}$ with $N=50$ ) for Brownian displacements (for a Gaussian distribution $P(\Delta x)$ with variance $\sigma^{2}$, the expected variance of $(\Delta x)^{2}$ amounts to $2 \sigma^{4}$, giving a relative standard deviation of $\sqrt{2 \sigma^{4}} / \sigma^{2}$. Then averaging over $N$ samples, this quantity reduces with a factor $1 / \sqrt{N}$ ). Importantly, this demonstrates that the different EGs in Hmec-1 cells experience different microenvironments.

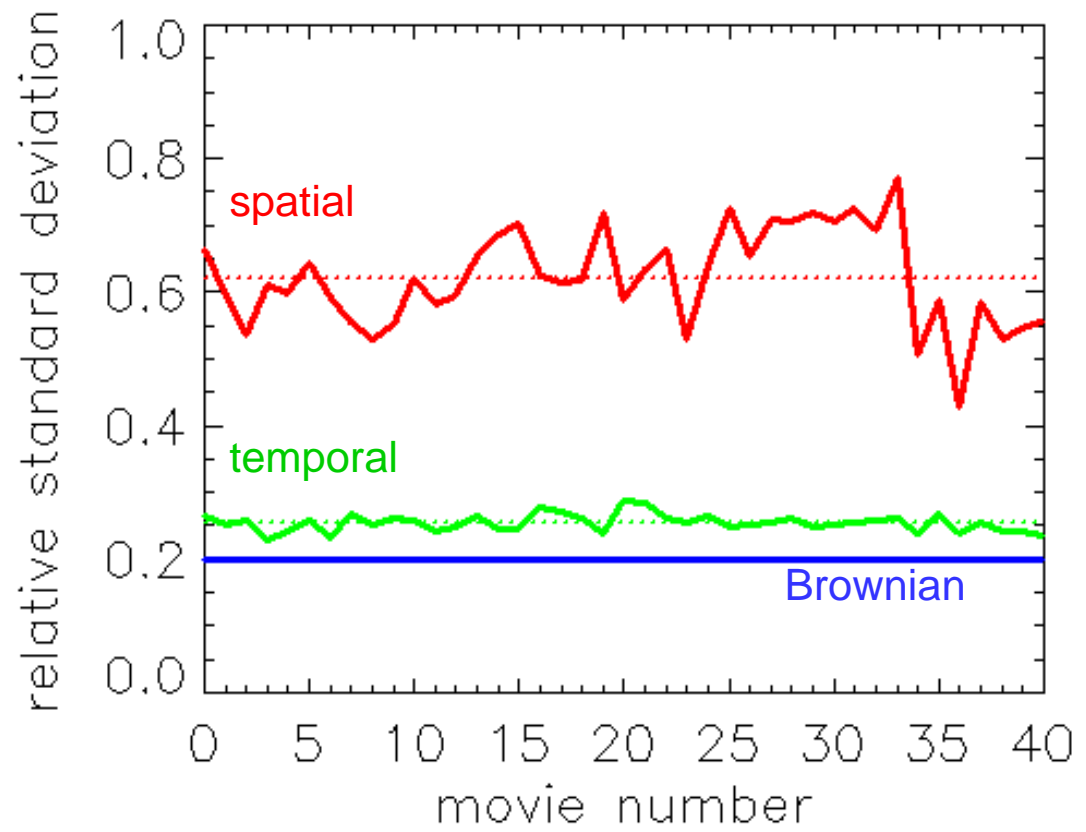

Fig.3.8: Relative standard deviations in the iMSD amplitude of EGs in Hmec-1 cells, calculated in two different manners. Spatial: c.f. Eq. 3.24. Temporal: c.f. Eq. 3.21. Dotted lines indicate averages over the 41 movies. For Brownian particles in Newtonian liquids, both averages coincide at 0.20. 
We also studied the relative standard deviation due to temporal variations in the iMSD of the same particle in more detail. If the relative standard deviation calculated from the time dependence is due to more than stochastic fluctuations alone, which is indeed suggested $(0.26>0.20)$, then a certain time dependence does exist. In that case, the calculated $\sigma_{r e l}$ should increase with the duration of the trajectory (as explained in Sec. 3.6). To examine this time dependence for the EGs, we sorted all non-segmented trajectories according to their length $l$ (see Fig. $3.5)$, and computed $\sigma_{\text {rel }}(l)$ by averaging over all 41 movies. We found that $\sigma_{\text {rel }}(l)$ gradually evolved from 0.20 at a track duration of $10 \mathrm{~s}$, to 0.26 for a duration of $150 \mathrm{~s}$. This indicates that at time scales up to $10 \mathrm{~s}$, the iMSD changes are rather small indeed, and that also the increase in temporal heterogeneity over $150 \mathrm{~s}$ is still modest. Apparently the time needed for important changes is indeed $>>150$ $\mathrm{s}$, as was also suggested from the time autocorrelation function.

\subsection{Discussion}

\subsubsection{Implications for particle tracking studies}

A method for characterizing spatial and temporal heterogeneity in particle dynamics was applied to endogenous granules in living Hmec-1 cells. We found appreciable local variations in iMSD but no systematic dependence on intracellular location (Fig. 3.4b). An important implication hereof, is that the precise distribution of the probes inside the cytoplasm is not crucial for observing a dynamic behavior that is representative for the whole cell. As long as the number of probes is high enough to sample the distribution of microenvironments, the variations in MSD will be averaged out. This means that it is justified to analyze the response of cells e.g. to drug treatments via the standard (i.e. ensemble averaging) particle tracking methods, even if the treated and untreated cells (and hence also their intracellular particle distributions) are different.

Also the absence of important transitions in the MSD of single particles, at least for durations up to $150 \mathrm{~s}$ (Figs. 3.7 and 3.8) has an important implication. It means that individual dynamic behaviors observed in this time window can be considered without an obvious need for trajectory segmentation. In other words, each particle will reflect an individual dynamics, which can be measured and analyzed directly from its MSD-vs.-lag time dependence, regardless of trajectory duration. These considerations will be further used in Section 3.5.2, in which we will analyze distributions of both the amplitude $A$ and the initial exponent $\alpha$ obtained from individual trajectories. 


\subsubsection{Application of individual trajectory analysis}

In this section we follow up on the finding that the heterogeneity in the iMSDs of our Hmec-1 cells is primarily due to differences between (the local environments of) particles. In other words, both the intracellular location of the particle and the integration time used for measuring the iMSD are relatively unimportant. Hence it is sufficient to consider lumped distributions of iMSDs. Besides EGs we here also consider BIPs as described in Sec 2.2. For these particles, less data could be obtained by particle tracking, due to phototoxic effects of the laser illumination (showing within minutes of exposure). Yet, graphs similar to Figs. 3.4 and 3.8 suggested that also for these probes, the spatial heterogeneity was dominant.
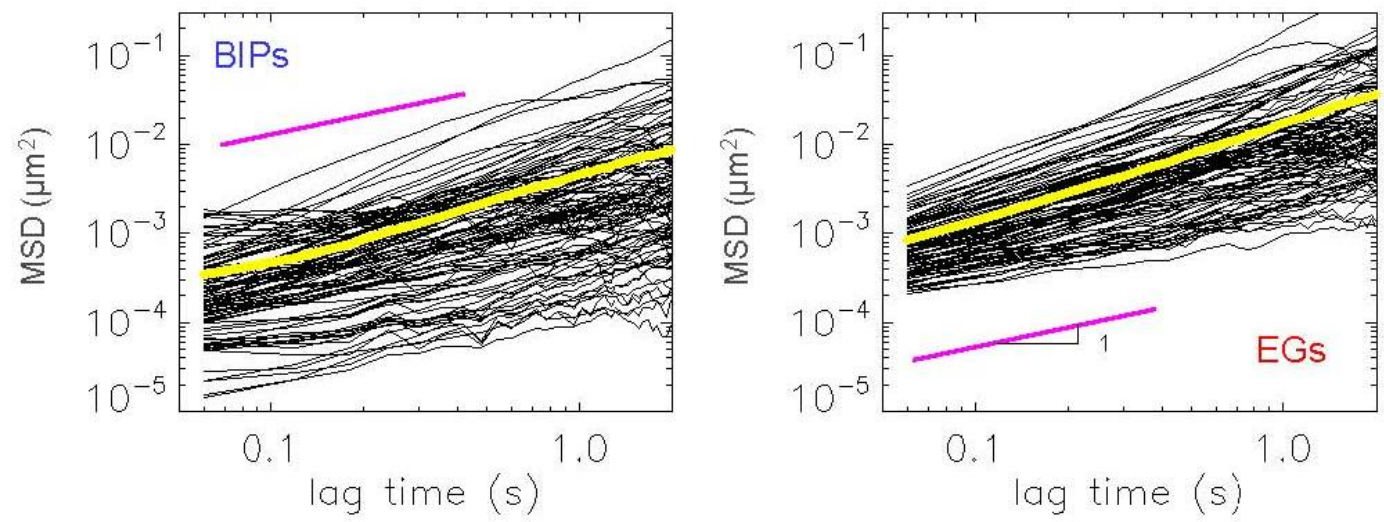

Fig.3.9: Representative set of 100 iMSD functions (based on trajectory segments c.f. Eq. 3.5) for BIPs (left) and EGs (right) in Hmec-1 cells. Average MSDs (c.f. Eq. 3.2) are shown in yellow. Solid magenta lines indicate a power-law exponent of 1 .

In Fig. 3.9 we show typical sets of iMSDs (based on segmented trajectories) of BIPs and EGs in (here non-confluent) Hmec-1 cells. Similar to Fig 4, broad amplitude distributions are found, for the BIPs even more than for the EGs. It is also apparent for both probe types that the (log-log) slopes show variations from particle to particle. In addition, some iMSDs appear noisy. This applies mostly to the BIPs for which the particle displacements are sometimes rather small.

Fitting power laws to the first 3 points of the iMSD functions, we obtained for each trajectory segment an amplitude $A$ and a power-law exponent $\alpha$, which we then collected into histograms. To compare histograms obtained for different probe/matrix combinations, they have to be brought onto a common (i.e. reduced) scale. For the dimensionless $\alpha$ this was not needed. For the amplitude $A$ this was achieved by subtracting $\langle\ln (A)\rangle$ from the distributed values of $\ln (A)$. The 
results shown in Fig. 3.10 are striking. First, the amplitude histograms do not resemble a simple lognormal distribution as found in [16, 39-41]. This applies most strongly to BIPs, for which it could already be seen from Fig. 3.9 that more than one type of particle dynamics occurs. For EGs the correspondence is better.

Also the power-law exponent $\alpha$ shows a remarkably broad distribution. It is now apparent that while the ensemble averaged MSDs for BIPs and EGs are close to simple behaviors (the average $\alpha$ being close to 0 and 1 respectively), in fact a significant variety in the dynamic behavior occurs for both probes. This holds the most strongly for the EGs, where it is suggested that besides diffusive, both suband superdiffusive behaviors occur. A biophysical interpretation of the differences between EGs and BIPs will be given elsewhere [37, 38].
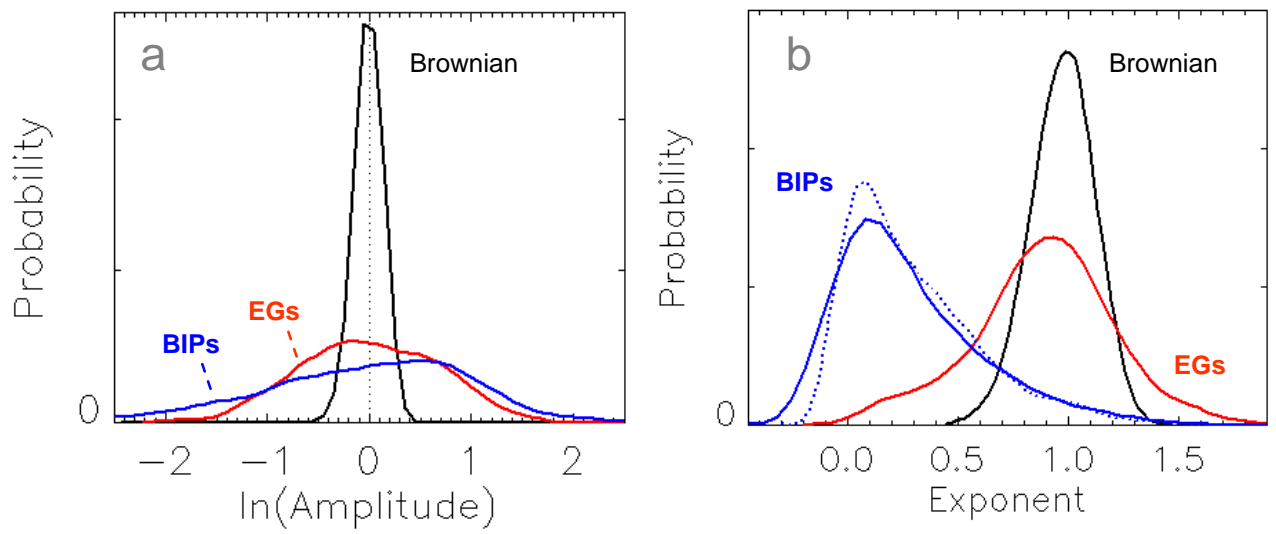

Fig. 3.10: Probability distributions for the logarithm of amplitude (InA) and power-law exponent $(\alpha)$ measured at $\tau \approx 0.1 \mathrm{~s}$, for two probe particles (EGs and BIPs) in individual Hmec-1 cells. For comparison, also the case of monodisperse particles in a Newtonian liquid is included. Distributions for $\ln A$ have been centered on zero. For BIPs the distribution of $\alpha$ is also shown for segmentation into blocks of 200 steps (dotted line).

We now consider some statistical aspects in the analysis of histograms like Fig $3.10 \mathrm{a}$ and $\mathrm{b}$. First of all we remark that dividing trajectories into segments of standard length is recommended when comparing such histograms. One reason for this is that it standardizes the broadening that takes place due to the finite trajectory lengths. This point is most clearly illustrated by the distributions obtained from the Brownian Dynamics simulation. Here each particle had been given the same diffusivity, implying that the expectation values for $A$ and $\alpha$ were exactly the same. Yet distributions are observed. For longer trajectory segments, these distributions become sharper. For our EGs in Hmec-1 cells studied with our 
camera, 50 steps per segment was an optimal choice. Choosing $N>50$ would have meant exclusion of a substantial fraction of the shortest trajectories from the analysis (see Fig. 3.5), possibly giving biased results, since in systems with heterogeneous dynamics the 'faster' particles generally have shorter trajectories [42]. A second reason for segmentation into standard blocks is that this can compensate for bias due to the different frequencies at which 'slow' and 'fast' particles leave and re-enter the focal plane of the microscope (and hence create 'new' trajectories [35]).

We attribute the occurrence of negative values of $\alpha$ for BIPs in Fig. 3.10b, to statistical broadening around an average $\alpha$ that is close to 0 . To corroborate this, we reanalyzed the BIPs trajectories after segmentation into blocks of 200 steps. As can be seen from fig. $3.10 \mathrm{~b}$, the occurrence of negative values has indeed become smaller. Following the same logic, a similar question could be posed about the significance of the apparently superdiffusive behavior $(\alpha>1)$ displayed by a fraction of the EGs. However, in this case a comparison can be made with the curve for the Brownian system, for which the average $\alpha$ is approximately the same. For the latter system, the chance of finding $\alpha>1.4$ for an iMSD based on 50 time steps is very small. Yet for EGs a substantial fraction of trajectories with $\alpha>1.4$ is still observed. This indicates that for EGs there exist superdiffusive behaviors, which are however hidden under an apparently simple diffusive overall MSD. This finding is qualitatively in line with the results of [14, 43].

\subsubsection{Comparison with other particle tracking methods}

The analysis method presented in this paper provides a straightforward extension of 1PMR. As such it also shares advantages and drawbacks of 1PMR. Depending on the selected probes and the embedding material of interest, the MSD function can reflect viscoelastic properties and/or probe-matrix interactions. In 'materials' like living cells, there may also be an additional contribution to the particle dynamics by ATP-dependent driving forces. Consequently, different (combinations of) properties can be studied with 1PMR. Knowledge of the chemistry between the probe and the matrix and/or the ability to eliminate nonthermal driving forces can then simplify interpretation. In this respect, living cells provide the biggest opportunities and challenges. This is however not limited to 1PMR. Also in 2PMR the particle dynamics can no longer be related directly to the intracellular rheology if non-thermal driving forces are acting.

What makes 2PMR unique is that it does not suffer from a lack of knowledge on the probe-matrix interactions, due to the fundamentally different measurement principle. In 2PMR one measures the correlated vectorial displacements of two 
particles, caused by the transmission of strain through the effective medium in between. For interparticle distances large compared to the typical size of a microenvironment, these correlations become insensitive to the microenvironments of the individual probes. Consequently, even in case of local probe-matrix interactions (like adhesion or repulsion, which can cause changes in the local microstructure), the measurement will reflect properties of the medium, that have been averaged over large lengthscales. While this aspect makes 2PMR more comparable to macroscopic rheology, it also makes 2PMR less suited for studying spatial heterogeneities. For example it may be found that $D_{r r}(r)$ does not scale anymore as $\sim 1 / r$, but then other methods will be needed for further inspection of heterogeneities.

Our method is hence complementary to both $1 \mathrm{PMR}$ and 2PMR in their standard application, neither of which reveals detailed information about spatial heterogeneity. Illustrations of spatial heterogeneity, both quantitatively (i.e. iMSD amplitude) and qualitatively (i.e. both sub and superdiffusivity) were given in this paper. In principle this analysis could also be taken further, using software to detect and analyze sub-groups of particles separately, based on their iMSD. Such an approach was already successfully applied to materials containing mechanically distinct (micro) phases [25].

A similar argument holds for temporal heterogeneity. Standard applications of 1PMR and 2PMR are not suited for detecting transitions from one dynamic behavior to the other by the same particle. However with trajectory segmentation as in Eq. 3.5 and variance analysis as in Eq. 3.21, it should be possible to detect for example the occurrence of intermittent dynamics [20,44], even if this occurs for only a small fraction of the particles.

\subsection{Conclusions and Outlook}

In this paper, we addressed spatiotemporal heterogeneity in the dynamics of endogenous lipid granules in living Hmec-1 cells. Careful analysis of large sets of individual particle MSDs, considering distributions over time as well as over particle populations, allowed to conclude that at the timescales pertinent to our experiments $(150 \mathrm{~s})$, particles can be distinguished according to their dynamic behavior. This allowed straightforward interpretation of distributions for the amplitude and power-law exponent. It thus became clear that not only the motion amplitude but also the type of dynamics showed considerable heterogeneity.

The implication hereof for obtaining a reliable ensemble averaged MSD, is that many particles per cell and/or trajectories consisting of many steps are needed. If 
(like in our case) spatial correlations between the different particles are absent, then the intracellular distribution of the particles will not have to be taken into account. The important implication of this outcome is that ensemble averaged MSDs measured in cells of the same type but not the very same cell, can still be meaningfully compared. This opens up the road to diagnosing living cells (e.g. before and after pharmacological interventions) via their MSD, even if the treated and untreated cells are not the same.

Finally we conclude that the statistical tools that were used to analyze spatiotemporal heterogeneity, should be equally applicable to a variety of other materials in which such heterogeneities occur. Measurements of correlation distances and times will require very large datasets. But even if these are not available, a straightforward analysis of relative variances could already provide a quick 'fingerprint' of heterogeneity.

\section{Acknowledgements}

This research was carried out in the "Cell Stress" Strategic Research Orientation of the MESA+ institute for nanotechnology. We thank Dirk van den Ende for stimulating discussions on particle tracking.

\section{References}

1 T. A. Waigh, Reports on Progress in Physics 68, 685 (2005).

2 D. Weihs, T. G. Mason, and M. A. Teitell, Biophysical Journal 91, 4296 (2006).

3 T. G. Mason, Rheologica Acta 39, 371 (2000).

$4 \quad$ S. R. Heidemann and D. Wirtz, Trends in Cell Biology 14, 160 (2004).

5 T. P. Kole, Y. Tseng, I. Jiang, J. L. Katz, and D. Wirtz, Molecular Biology of the Cell 16, 328 (2005).

6 P. Panorchan, J. S. H. Lee, B. R. Daniels, T. P. Kole, Y. Tseng, and D. Wirtz, Cell Mechanics 83, 115(2007).

7 P. Panorchan, J. S. H. Lee, T. P. Kole, Y. Tseng, and D. Wirtz, Biophysical Journal 91, 3499 (2006).

8 Y. Tseng, T. P. Kole, S. H. J. Lee, and D. Wirtz, Current Opinion in Colloid \& Interface Science 7, 210 (2002).

9 Y. Tseng, T. P. Kole, and D. Wirtz, Biophysical Journal 83, 3162 (2002).

10 S. Yamada, D. Wirtz, and S. C. Kuo, Biophysical Journal 78, 1736 (2000).

11 J. C. Crocker and B. D. Hoffman, in Cell Mechanics, 2007), Vol. 83, p. 141.

12 J. C. Crocker, B. D. Hoffman, G. Massiera, and K. Miranda, Biophysical Journal 86, 59A (2004). 
13 B. D. Hoffman, G. Massiera, K. M. Van Citters, and J. C. Crocker, Proceedings of the National Academy of Sciences of the United States of America 103, 10259 (2006).

14 A. W. C. Lau, B. D. Hoffman, A. Davies, J. C. Crocker, and T. C. Lubensky, Physical Review Letters 91, 4 (2003).

15 G. Massiera, K. M. Van Citters, P. L. Biancaniello, and J. C. Crocker, Biophysical Journal 93, 3703 (2007).

16 K. M. Van Citters, B. D. Hoffman, G. Massiera, and J. C. Crocker, Biophysical Journal 91, 3946 (2006).

17 C. Wilhelm, Physical Review Letters 101, 4 (2008).

18 C. Wilhelm, A. Cebers, J. C. Bacri, and F. Gazeau, European Biophysics Journal with Biophysics Letters 32, 655 (2003).

19 P. Bursac, B. Fabry, X. Trepat, G. Lenormand, J. P. Butler, N. Wang, J. J. Fredberg, and S. S. An, Biochemical and Biophysical Research Communications 355, 324 (2007)

20 P. Bursac, G. Lenormand, B. Fabry, M. Oliver, D. A. Weitz, V. Viasnoff, J. P. Butler, and J. J. Fredberg, Nature Materials 4, 557 (2005).

21 J. L. McGrath, J. H. Hartwig, and S. C. Kuo, Biophysical Journal 80, 2487 (2001).

22 M. T. Valentine, Z. E. Perlman, M. L. Gardel, J. H. Shin, P. Matsudaira, T. J. Mitchison, and D. A. Weitz, Biophysical Journal 86, 4004 (2004).

23 D. Mizuno, C. Tardin, C. F. Schmidt, and F. C. MacKintosh, Science 315, 370 (2007).

24 J. C. Crocker, M. T. Valentine, E. R. Weeks, T. Gisler, P. D. Kaplan, A. G. Yodh, and D. A. Weitz, Physical Review Letters 85, 888 (2000).

25 M. T. Valentine, P. D. Kaplan, D. Thota, J. C. Crocker, T. Gisler, R. K. Prud'homme, M. Beck, and D. A. Weitz, Physical Review E 6406 (2001).

26 D. Weihs, M. A. Teitell, and T. G. Mason, Microfluidics and Nanofluidics 3, 227 (2007).

27 C. Oelschlaeger, N. Willenbacher, and S. Neser, Progr. Colloid Polym Sci. DO10.1007/2882 2008 083I

28 F. K. Oppong and J. R. de Bruyn, J. Non-Newtonian Fluid. Mech. 142, 104 (2007).

29 F. K. Oppong, L. Rubatat, B. J. Frisken, A. E. Bailey, and J. R. de Bruyn, Physical Review E 73, 9 (2006).

30 J. Y. Xu, Y. Tseng, C. J. Carriere, and D. Wirtz, Biomacromolecules 3, 92 (2002).

31 W. K. Kegel and A. van Blaaderen, Science 287, 290 (2000).

32 T. Moschakis, B. S. Murray, and E. Dickinson, Langmuir 22, 4710 (2006). 
33 J. H. Dangaria and P. J. Butler, American Journal of Physiology-Cell Physiology 293, C1568 (2007).

34 S. S. Rogers, T. A. Waigh, and J. R. Lu, Biophysical Journal 94, 3313 (2008).

35 http://www.physics.emory.edu/ weeks/idl/

36 J. C. Crocker and D. G. Grier, Journal of Colloid and Interface Science 179, 298 (1996).

37 S.A.Vanapalli, Y. Li, F. Mugele, M.H.G. Duits. On the origins of the universal dynamics of endogenous granules in mammalian cells, Molecular and Cellular Biomechanics, 150, 1-16, 2009

38 Y. Li , S.A.Vanapalli, F. Mugele, M.H.G. Duits. Dynamics of Ballistically Injected Latex Particles in Living Human Endothelial Cells, Biorheology 46(4), 309-321 (2009)

39 B. Fabry, G. N. Maksym, J. P. Butler, M. Glogauer, D. Navajas, N. A. Taback, E. J. Millet, and J. J. Fredberg, Physical Review E 68, 18 (2003).

40 B. Fabry, G. N. Maksym, S. A. Shore, P. E. Moore, R. A. Panettieri, J. P. Butler, and J. J. Fredberg, Journal of Applied Physiology 91, 986 (2001).

41 N. Desprat, A. Richert, J. Simeon, and A. Asnacios, Biophysical Journal 88, $2224(2005)$

42 T. Savin and P. S. Doyle, Physical Review E 76 (2007).

43 A. Caspi, R. Granek, and M. Elbaum, Physical Review Letters 85, 5655 (2000).

44 Z. Bomzon, M. M. Knight, D. L. Bader, and E. Kimmel, Journal of Biomechanical Engineering-Transactions of the Asme 128, 674 (2006). 


\title{
Chapter 4
}

\section{On the Origins of the Universal Dynamics of Endogenous Granules in Mammalian cells}

\begin{abstract}
Endogenous granules (EGs) that consist of lipid droplets and mitochondria have been commonly used to assess intracellular mechanical properties via multiple particle tracking microrheology (MPTM). Despite their widespread use, the nature of interaction of EGs with the cytoskeletal network and the type of forces driving their dynamics - both of which are crucial for the interpretation of the results from MPTM technique - are yet to be resolved. In this report, we study the dynamics of endogenous granules in mammalian cells using particle tracking methods. We find that the ensemble dynamics of EGs is diffusive in three types of mammalian cells (endothelial cells, smooth muscle cells and fibroblasts), thereby suggesting an apparent universality in their dynamical behavior. Moreover, in a given cell, the amplitude of the meansquared displacement for EGs is an order of magnitude larger than that of injected particles. This observation along with results from ATP depletion and temperature intervention studies suggests that cytoskeletal active forces drive the dynamics of EGs. To elucidate the dynamical origin of the diffusive-like nonthermal motion, we consider three active force generation mechanisms molecular motor transport, actomyosin contractility and microtubule polymerization forces. We test these mechanisms using pharmacological interventions. Experimental evidence and model calculations suggest that EGs are intimately linked to microtubules and that microtubule polymerization forces drive their dynamics. Thus, endogenous granules could serve as non-invasive probes for microtubule network dynamics in mammalian cells.
\end{abstract}

This chapter has been published in Molecular and Cellular Biomechanics 150, 1-16 (2009) 


\subsection{Introduction}

Cellular function is intimately linked to their mechanical properties $(1,2)$. For example, the ability of cells to crawl and migrate is influenced by their global mechanical properties as quantified by their frequency dependent shear moduli. In addition, cells constantly modify their local mechanical properties by remodeling their internal microstructure (3). This precise control over cellular mechanical behavior is orchestrated by the cytoskeleton - a multifunctional body consisting of dense networks of biopolymers that is being dynamically modulated by a number of active processes driven by a myriad of cytoskeletal proteins and ATP (4).

Recently, significant advances have been made in the understanding of the mechanical behavior of the living cytoskeleton using a variety of techniques including optical tweezers (5), magnetic twisting cytometry (6), atomic force microscopy (7), particle-tracking microrheology (8) and microplate rheometry (9). Among these techniques multiple particle tracking microrheology (MPTM) is a versatile method as both the local and global cellular mechanical properties can be simultaneously determined using probe particles $(10,11)$. In the literature (12-17) naturally present endogenous granules (usually lipid droplets and mitochondria) have been widely used to assess intracellular rheology. In spite of their widespread use, knowledge of the nature of interaction of endogenous granules (EGs) with the cytoskeleton, that underpins the MPTM method is lacking. Recent evidence suggests that the EGs are sensitive to non-thermal intracellular stress fluctuations (11). However, neither the microscopic origin of these active forces nor the concomitant cytoskeletal biopolymers that drive the motion of endogenous granules have been identified. In this study, we investigate the motion of endogenous granules under a variety of pharmacological conditions and report the first evidence that the dynamics of endogenous granules is intimately linked to the microtubule polymerization forces.

\subsection{Materials and methods}

\subsubsection{Cell Culture and intracellular probes}

Human microvascular endothelial cells (HMECs), human umbilical vein smooth muscle cells and COS-7 African green monkey kidney fibroblasts were cultured at $37^{\circ} \mathrm{C}$ in a humidified $5 \% \mathrm{CO}_{2}$ environment. Cells were plated on a Delta $\mathrm{T}$ culture dish (Bioptechs, Butler, PA, USA), precoated with fibronectin (100 $\mu \mathrm{g} / \mathrm{mL}$ solution). The endogenous granules were identified to be lipid droplets and mitochondria by staining with nile red and rhodamine dyes respectively. The 
mean diameter of the granules was assessed to be $\approx 0.5 \mu \mathrm{m}$. The injected particles were carboxylated fluorescent polystyrene beads of $200 \mathrm{~nm}$ in size and were introduced ballistically into the cells using a Biolistic gun (He/PS 1000, BioRad) according to the protocol of Panchoran et al. (18).

\subsubsection{Interventions}

All drugs were purchased from Sigma Aldrich, except blebbistatin (Tebu-bio, Belgium). Drug intervention studies were performed only with endothelial cells and the following drugs were used: Latrunculin A (200 nM, 2hrs), Blebbistatin (50 $\mu \mathrm{M}, 2 \mathrm{hrs})$, Jasplakilonide $(25 \mathrm{nM}, 1 \mathrm{hr})$, Nocodazole $(5 \mu \mathrm{M}, 3 \mathrm{hrs})$ and Cephalomannine (taxol, $100 \mu \mathrm{M}, 1 \mathrm{hr}$ ). For ATP depletion, 2-D-deoxyglucose and sodium azide at final concentrations of $50 \mathrm{mM}$ and $0.05 \%$ were respectively used for 30 mins. To study temperature effects, the Bioptechs heating system was used to modulate the temperature of the cells between $37{ }^{\circ} \mathrm{C}$ and $24{ }^{\circ} \mathrm{C}$ allowing for a $30 \mathrm{~min}$ waiting period. For all drug intervention experiments ten endothelial cells were tracked over a period of 2-3 hrs.

\subsubsection{Multiple particle tracking experiments}

Probe particles were visualized using a spinning-disk inverted confocal microscope (UltraView LCI 10, Perkin Elmer, Cambridge, UK). Endogenous granules were imaged under phase contrast mode using a $100 \mathrm{X}$ (NA 1.3) objective, while ballistically injected particles were visualized under fluorescence mode. Approximately, 2500 images were recorded with a 12-bit CCD camera (Hamamatsu, Bridgewater, NJ) at $\sim 17$ frames per second for a typical duration of 150 seconds. The spatial resolution corresponding to the images was $0.13 \mu \mathrm{m}$ per pixel. In each cell, 50-100 EGs and 15-40 BIPs were tracked. The particles were identified using the publicly available particletracking code based on the paper by Crocker and Grier (19), written and extended in Interactive Data Language (IDL, Visual Information Solutions, Boulder, CO) by several authors (see acknowledgement).

We now discuss the errors associated with our particle-tracking experiments. The error in our particle displacements was measured to be $\approx 6-10 \mathrm{~nm}$ by tracking immobilized particles. To assess the bias associated with sampling more of the trajectories corresponding to slowly moving particles, we performed block analysis of the images. A single run of 2500 images containing EGs in an endothelial cell was split up into blocks of 300 images and the calculated ensemble-averaged mean-squared displacement was found to be indistinguishable for the various block data sets. To assess the extent of inaccuracy introduced in our data by slow processes (e.g. cell crawling), we 
recorded four consecutive movies of EGs in the same endothelial cell over a period of 30 minutes. We found the mean squared displacement (MSD) curves to exhibit similar temporal response with amplitude variation of $\approx 10-20 \%$.

We associate molecular motor transport with superdiffusive behavior (20-22) i.e. where the power-law exponent corresponding to MSD curves is greater than unity. To identify molecular motor transport (i.e. superdiffusive) behavior in the dynamics of EGs we gathered 10000 single particle (granule) trajectories. We calculated the power-law exponent corresponding to single particle MSDs and generated power-law exponent distributions. We assessed the statistical significance of these distributions by comparing it against Brownian particle simulations (23). In our simulations for Brownian systems, power-law exponents below or above 1.0 were obtained occasionally for trajectory lengths comparable to our experiments, probably due to finite trajectory lengths. This finding indicates that based on the power-law exponent alone, it is not generally possible to tag individual particle trajectories as superdiffusive. We therefore generated power-law exponent distributions and in instances where we observed a portion of the distribution to lie outside the Brownian particle distribution, we attributed it to be statistically significant.

\subsection{Results \& discussions}

4.3.1 Universal diffusive dynamics of endogenous granules are driven by cytoskeletal active forces

Figure 4.1 shows the ensemble mean-squared displacement of endogenous granules analyzed from ten different endothelial cells. We find that in spite of cell to cell amplitude variation in MSD, the temporal response shows a powerlaw slope close to 1 . We use the term diffusive to describe this behavior because of the powerlaw slope of unity. We also tracked endogenous granules in other cell types to verify if this diffusive behavior persists. Figure 4.2 reports the mean-squared displacement (MSD) of endogenous granules in three different mammalian cell lines - endothelial cells $(\alpha=0.90 \pm 0.01)$, smooth muscle cells $(\alpha=0.83 \pm 0.01)$ and fibroblasts $(\alpha=0.94 \pm 0.01)$. Here $\alpha$ is the exponent derived from power-law fit to the data. Remarkably, we find that the dynamics of EGs in all three types of cells is nearly diffusive over the time-scales probed. Similar diffusive behavior has also been observed in macrophages and carcinoma cells (11), but in yeast (14) and amoeba (17), subdiffusive behavior was found. These observations suggest that the diffusive dynamics of endogenous granules might be a universal characteristic of mammalian cells. 


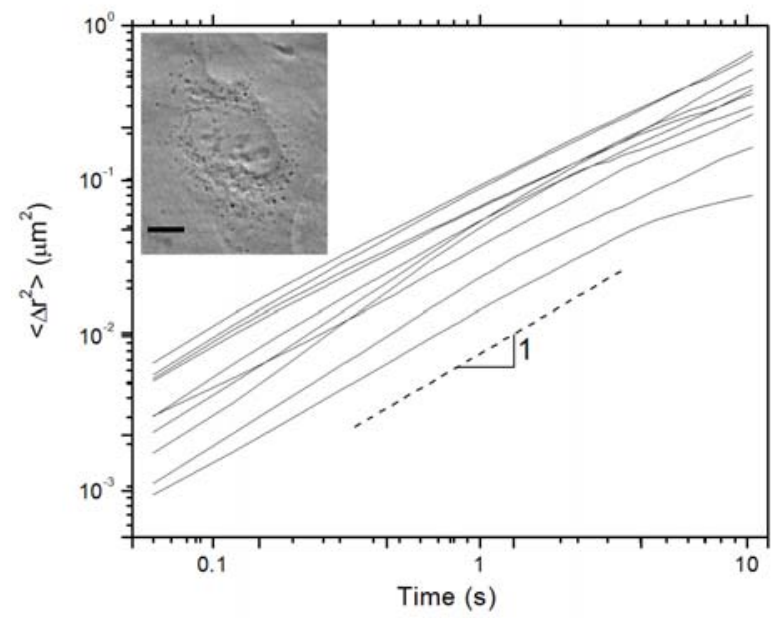

Fig. 4.1: Ensemble dynamics of endogenous granules in endothelial cells (HMEC-1) at $37^{\circ} \mathrm{C}$. The lines are the ensemble-averaged mean-squared displacement of EGs for ten individual cells. Inset shows phase contrast image of an endothelial (HMEC-1) cell with the dark objects being endogenous granules. Scale bar is $10 \mu \mathrm{m}$.

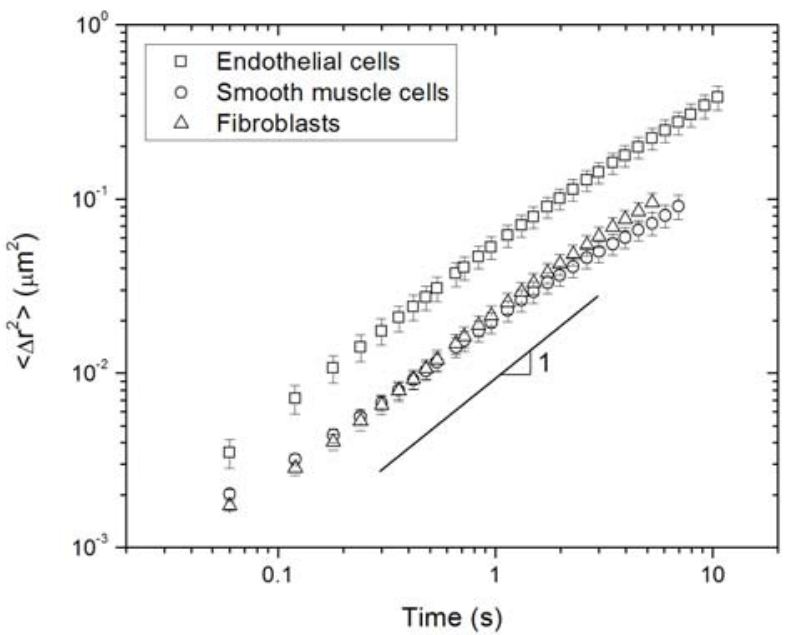

Fig. 4.2: Mean squared displacement of endogenous granules versus lag time at $37{ }^{\circ} \mathrm{C}$ for three different kinds of mammalian cells. The symbols represent the average over $N=10,5$ and 6 cells for endothelial cells, smooth muscle cells and fibroblasts respectively. The error bars are standard errors. 
To assess whether the observed universal diffusive behavior of endogenous granules is driven by passive (thermal) or active (non-thermal) forces we used two approaches. First, we studied the effect of active motion interventions lowering the temperature and ATP depletion - on the ensemble dynamics of EGs. We find that both the treatments reduce the amplitude of the MSD (see Fig. 4.3) to similar values, with temperature reduction and ATP depletion yielding $\alpha=$ $0.76 \pm 0.04$ and $0.66 \pm 0.03$ respectively. These results suggest that cytoskeletal active forces play a central role in determining the dynamics of endogenous granules.

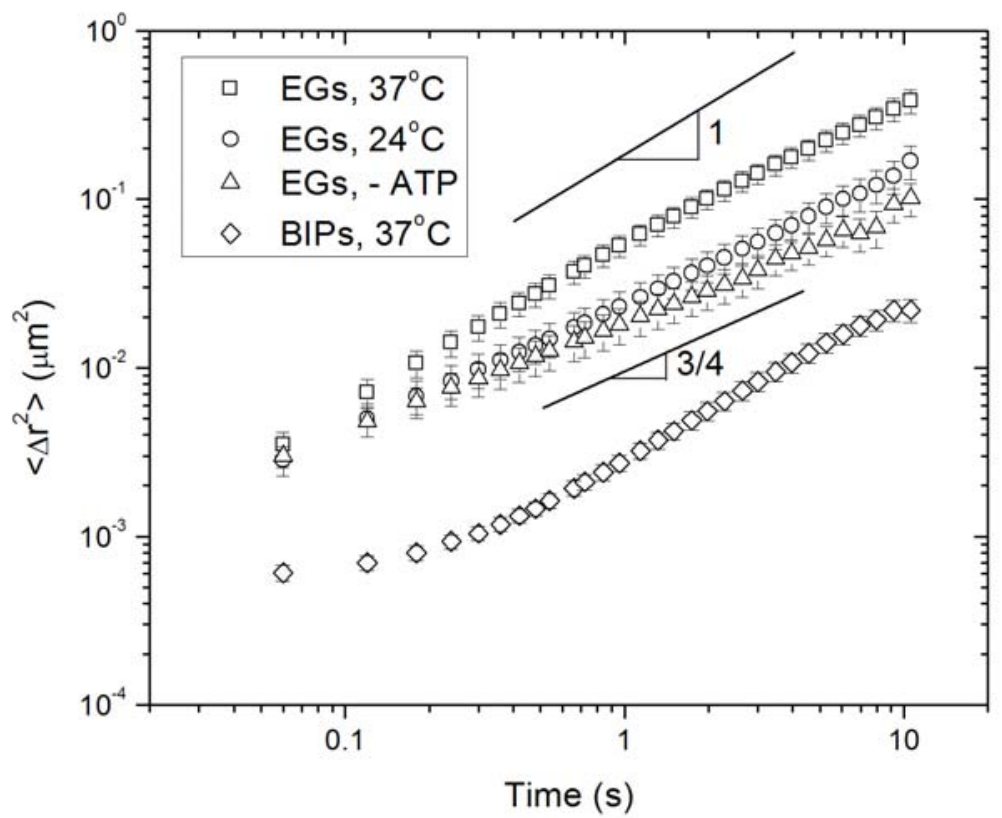

Fig.4.3: Influence of ATP depletion and reduction in temperature on the ensemble dynamics of endogenous granules in endothelial cells. Also shown is the mean-squared displacement of ballistically injected particles. The error bars are standard errors calculated from data acquired on ten endothelial cells.

Second, we performed independent particle tracking experiments with ballistically injected particles (BIPs) and compared their MSD against that of EGs. Remarkably, we find that, although BIPs are about half the size of EGs, their amplitude of MSD is about an order of magnitude smaller than that of EGs, This amplitude difference persists in spite of cell-to-cell variability. To further validate these remarkable differences in the ensemble dynamics of EGs and BIPs, 
we analyzed an additional set of five cells containing both EGs and BIPs. Again, the large difference between the amplitudes of the MSDs for the two probes was observed. Since ballistically injected particles have been previously reported to behave as passive probes in living cells (18), we attribute the enhanced amplitude in the MSD of EGs compared to BIPs to active cytoskeletal forces.

4.3.2 Probing the origins of the non-equilibrium dynamics of endogenous granules

We now probe the origins of the diffusive dynamics of endogenous granules that appears to be a generic feature of mammalian cells and is being driven by nonequilibrium fluctuations. A major contributor to non-equilibrium motion in living cells are ATP-driven active processes (11). At the molecular level there could be many sources of ATP-driven activity in living cells, however from the perspective of cellular mechanical force generation, we consider three known candidates for intracellular active behavior.

4.3.2.1 Molecular motor driven transport

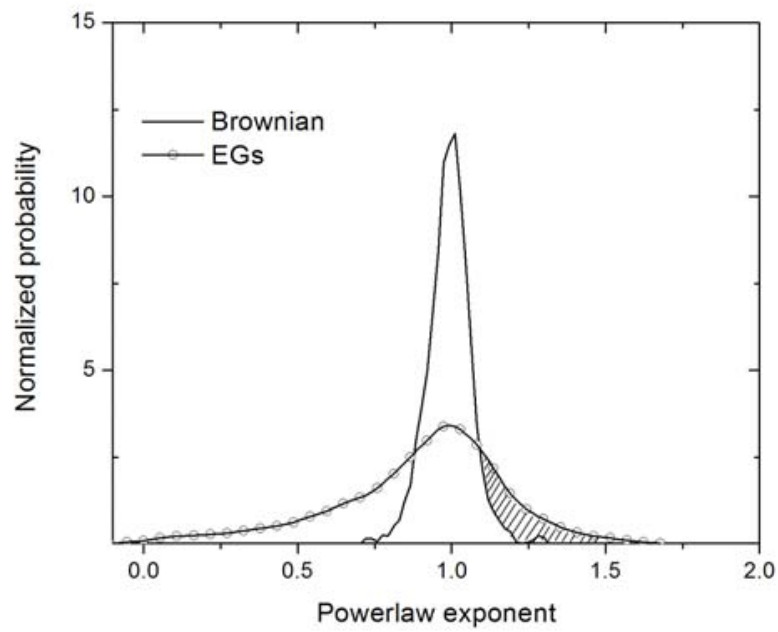

Fig.4.4: Probability distributions of the power-law exponents corresponding to single-particle trajectories for endogenous granules in endothelial cells compared against Brownian particles. The hatched area denotes the statistically significant population of EGs that show superdiffusive behavior.

Intracellular active transport arises from interaction of either kinesin and dynein motors with microtubules or myosins with actin filaments. In both cases, this type of cargo-transport behavior has been shown to result in superdiffusive 
behavior (20-22). We analyze the single-particle trajectories of EGs (c.f. Section 4.2.3) and indeed observe super-diffusive behavior as shown in Figure 4.4. However, the fraction of EGs exhibiting such intracellular transport is rather small. Eliminating the statistically significant superdiffusive trajectories $(\alpha>1.2$ as per fig.4.4) and re-analyzing the data (not shown), we still find the dynamics of EGs to be predominantly diffusive. Therefore it is unlikely that the ensemble dynamics of endogenous granules is being driven simply by intracellular transport coupled to motor protein activity.

\subsubsection{Actomyosin contractility}

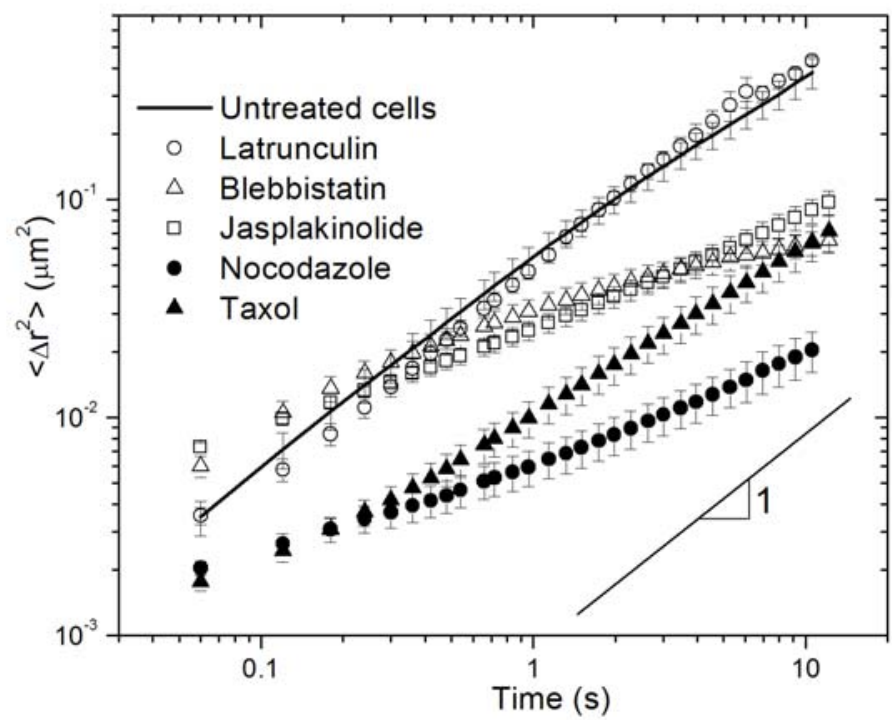

Fig. 4.5: Effect of specific pharmacological interventions on the dynamics of endogenous granules in endothelial cells.

Contractile mechanical forces are generated in living cells via actin-myosin interactions, where the myosin II motor cross-links and slides on actin filaments. Here we consider if such actin-myosin contractile forces are important for the observed diffusive dynamics of endogenous granules. This hypothesis has been motivated by a recent in vitro study (24) involving a composite network of actin filaments, microtubules and myosin II motors that has shown motor-induced non-thermal fluctuations to be diffusive-like. To test this hypothesis in vivo we subjected the endothelial cells to latrunculin, blebbistatin and jaspakilonide drug interventions. Latrunculin (LA) binds to actin monomers and inhibits the 
polymerization of actin filaments, implying that the associated actin binding proteins (including myosin isoforms) are presumably inactivated as well. Blebbistatin specifically disrupts the activity of the myosin II motor and jasplakinolide stabilizes the actin network by inducing actin polymerization.

In Figure 4.5 we show that the LA intervention does not significantly alter the MSD of endogenous granules, although from the cell morphology and immunofluorescene staining of the actin network (data not shown) we observe that LA was active and efficient. This observation suggests that the intracellular actin network does not affect the dynamics of endogenous granules. Contrary to the above conclusion, both blebbistatin and jasplakinolide interventions yield an MSD amplitude and slope that is different from that of EGs in the untreated cells. We reconcile this apparent discrepancy and discuss the importance of actinmyosin contractility for EG dynamics in Section 4.3.3.

\subsubsection{Microtubule bending forces}

Non-equilibrium microtubule bending modes in living cells could arise either from acto-myosin contractility (24) or from microtubule polymerization forces (25). Here, we consider the bending dynamics of microtubules driven by nonthermal forces as a possible source of diffusive dynamics of EGs. This hypothesis has been motivated by a recent direct observation of intracellular microtubule tip growth dynamics, which was found to be diffusive-like and ATP dependent (25). To test this hypothesis we subjected endothelial cells to nocodazole and taxol drug interventions. Nocodazole binds to beta-tubulin and prevents microtubule polymerization, while taxol promotes the assembly of stable microtubules from alpha and beta tubulin and inhibits their depolymerization. In Figure 4.5, we show that both nocodazole and taxol dramatically reduce the amplitude of MSD, indicating that microtubules are an important cytosleketal element affecting the dynamics of EGs.

\subsubsection{Microtubule polymerization dynamics drives the motion of endogenous granules in mammalian cells}

In the previous section, we assessed possible mechanistic origins of endogenous granule dynamics. In this section, we begin with two model calculations that support the notion that microtubules play a crucial role in determining the dynamics of EGs. The first model analysis estimates the amplitude of the MSD experienced by endogenous granules linked to thermally driven microtubules. The second model links actively driven microtubule bending dynamics to 
endogenous granule motion. We use this model subsequently to explain the observed effects of drug interventions (c.f. Fig. 4.5) on EG dynamics.

Monomer fluctuations due to thermal energy in a semiflexible polymer network have been shown both experimentally and theoretically $(26,27)$ to obey a unique scaling of the form

$$
\left\langle\Delta r_{o}^{2}\right\rangle=A t^{3 / 4}=0.41\left\lfloor\ln \left(\frac{\kappa t \ln (L / \pi a)}{4 \pi \eta a^{4}}\right)\right\rfloor \frac{k_{B} T}{\eta^{3 / 4} \kappa^{1 / 4}} t^{3 / 4}
$$

In Eqn (4.1) $k_{B} T$ is thermal energy, $\eta$ is solvent viscosity, $L$ is the polymer contour length, $a$ is the chain diameter and $\kappa$ is the bending rigidity. In our active motion intervention experiments, especially under temperature reduction to $24{ }^{\circ} \mathrm{C}$ (c.f. Figure 4.3), we observe a power-law exponent of $\sim 3 / 4$ for $\mathrm{EG}$ dynamics. Assuming EGs are linked to a semiflexible polymer network, we assess which of the semiflexible polymer networks in living cells - actin filament (AF), microtubule (MT) or intermediate filament (IF) networks - yield an estimate of the prefactor $\mathrm{A}$ that is comparable with temperature reduction experiment. For this calculation, we take $\eta=0.1 \mathrm{~Pa} . \mathrm{s}(2), \mathrm{L}=1 \mu \mathrm{m}$. The values of $\kappa$ for the AF, MT and IF are $7 \times 10^{-26}, 2 \times 10^{-23}$ and $4 \times 10^{-27} \mathrm{Nm}^{2}$ respectively $(1,28)$. The corresponding chain diameters are $2.5,15$ and $10 \mathrm{~nm}(1,28)$. We find that $A \approx 0.42,0.09$ and $0.52 \mu \mathrm{m}^{2} / \mathrm{s}^{3 / 4}$ for $\mathrm{AF}$, MT and IF networks respectively. From Fig. 4.3, we determine a prefactor of $0.02 \mu \mathrm{m}^{2} / \mathrm{s}^{3 / 4}$ that is closest to the predicted value for microtubules rather than for actin or intermediate filaments. Thus, this analysis suggests that endogenous granule motion is driven by microtubules. 

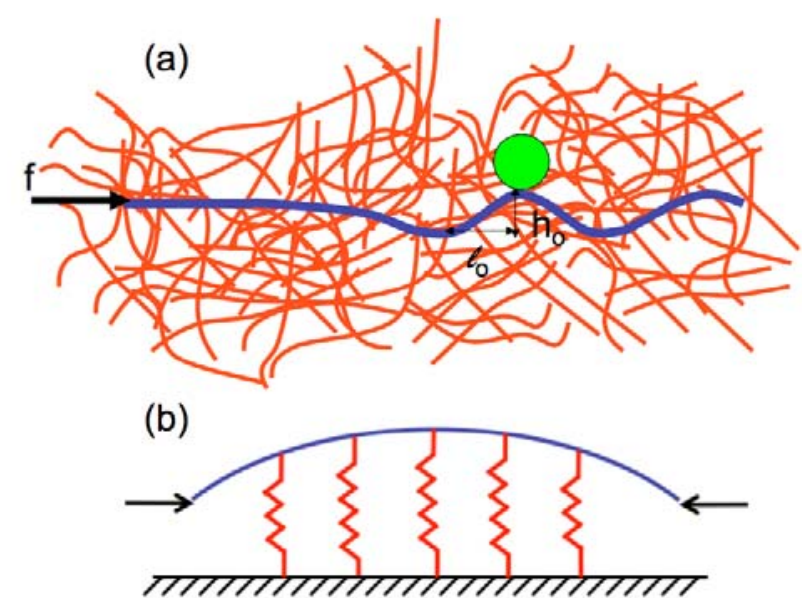

Fig. 4.6: (a) A schematic (not to scale) showing a microtubule (in blue) embedded in an elastic medium (in red) undergoing short-wavelength bending due to a motor-driven force, $f$ (modified from ref. 24). The endogenous granule (in green) is shown anchored to the microtubule. The characteristic lateral and transverse bending length scales are denoted by $l_{o}$ and $h_{o}$ respectively (b) A mechanical model showing how a localized bend in the microtubule could be elastically coupled to the surrounding network at the point of incipient buckling. Because of the coupling of the elastic medium, the characteristic wavelength for buckling will be shorter than the length of the rod.

We now estimate the magnitude of non-thermal forces driving EG dynamics assuming that EGs experience the transverse bending modes due to microtubule buckling as shown in Figure 4.6. Our analysis is based on recent literature models $(25,29-31)$ that consider a microtubule of bending rigidity, $\kappa$, undergoing buckling in an (isotropic, homogeneous and linear) elastic medium of modulus, $G$, due to a motor-driven compressional force, f. We briefly discuss this model for the sake of completeness. When a compressional point force due to a motor protein is imposed as shown in Figure 4.6, unlike classical long-wavelength Euler buckling the microtubule undergoes short-wavelength buckling. This is because, in the presence of an elastic medium, for the same end-to-end compression, the short wavelength buckling is energetically more favorable than long-wavelength Euler buckling. This microtubule buckling behavior can be described by a characteristic longitudinal and transverse length scales of order $l_{o}$ and $h_{\mathrm{o}}$ respectively. To link the compression force $\mathrm{f}$ to the microtubule 
deflections, we consider the energy associated with the buckling behavior. The bending energy along the length of the microtubule is given by

$$
\int \kappa\left(\frac{d^{2} u}{d x^{2}}\right)^{2} d x \sim \kappa\left(\frac{h_{o}}{l_{o}^{2}}\right)^{2} l_{o} \sim \kappa \frac{h_{o}^{2}}{l_{o}^{3}}
$$

where $\kappa \quad$ is the bending rigidity and $\mathrm{u}(\mathrm{x})$ is the transverse deflection as a function of the axial coordinate $\mathrm{x}$. At the point of incipient buckling, the surrounding elastic network resists the microtubule buckling as shown schematically by the springs in Fig. 4.6b. This competing elastic energy due to the surrounding matrix is given by

$$
\int G u^{2} d x \sim G h_{o}^{2} l_{o}
$$

At the onset of buckling this elastic energy of the matrix is of the order of the bending energy associated with the buckled microtubule i.e. $\kappa \frac{h_{o}^{2}}{l_{o}^{3}} \sim G h_{o}^{2} l_{o}$, which yields

$$
l_{o} \sim(\kappa / G)^{1 / 4}
$$

This estimate of $l_{o}$ corresponds to the characteristic wavelength associated with constrained buckling (29). Using typical values of $\kappa \approx 10^{-23} \mathrm{Nm}^{2}$ and $G \approx 1$ $\mathrm{kPa}$ reported in the literature $(28,32)$ we obtain $l_{o} \approx 1 \mu \mathrm{m}$. Now the active force, $\mathrm{f}$, can be estimated from these elastic energies (either Eqn (4.2) or Eqn (4.3)) as

$$
f \sim \frac{d E}{d u} \sim \frac{\kappa h_{o}}{l_{o}^{3}}
$$

By assuming that the EGs experience the transverse bending modes of the microtubules (see Fig. 4.6), we estimate $h_{\mathrm{o}}$ to be of the order of the root-mean squared displacement of EGs reported in Figure 4.2, i.e. $h_{o} \approx \sqrt{\left\langle\Delta r^{2}\right\rangle} \approx 0.1-1 \mu \mathrm{m}$. Thus, from Eqn (4.5), we obtain an estimate of active force $f \approx 1-10 \mathrm{pN}$, which is in accordance with typical forces exerted by motor-driven forces (33). Although this model, does not yield information on the specific nature of the active force it is likely that the source is either the actin-myosin contractility or microtubule polymerization forces, since both of them are capable of inducing microtubule bucking. Nevertheless, the model calculation indicates that microtubules play a crucial role in determining the dynamics of EGs.

According to the above model, using Eqns (4.4) and (4.5), it can be shown that

$$
\left\langle\Delta r^{2}\right\rangle \sim f^{2} / G^{3 / 2} \kappa^{1 / 2}
$$

Eqn (4.6) implies that for a given microtubule stiffness, the amplitude of MSD of EGs is determined by two competing parameters - the magnitude of the active 
force, $\mathrm{f}$, driving microtubule buckling and the elastic modulus, $\mathrm{G}$, of the medium resisting buckling. Within this framework of the model, we interpret the results of drug interventions with AF and MT network reported in Figure 4.5. With the disruption of the actin network by latrunculin, the amplitude of MSD of EGs remains unchanged implying that- (i) the source of the active driving force, $f$, does not arise from actin-myosin contractility, but probably from microtubule polymerization forces. Thus acto-myosin contractility is not important in influencing the dynamics of EGs (ii) The modulus of the elastic medium resisting microtubule buckling is not determined by the actin network. Although this conclusion may appear surprising, recently, two-point microrheology of epithelial cells has also shown that the actin network does not contribute significantly to the intracellular rheology (30).

With blebbistatin and jasplakinolide, we observe a decrease in the MSD amplitude of EGs. Since we concluded earlier that active driving force for EGs arises from microtubule polymerization forces, this suggests that both myosin motor inhibition (by blebbistatin) and actin stabilization (by jasplakinolide) enhances the stiffness $(\mathrm{G})$ of the underlying medium (c.f. Eqn (4.6)). Bursac et al. (6), also found reduced amplitude in the MSD under jasplakinolide intervention, of a bead attached to human airway smooth muscle cell cortex using magnetic twisting cytometry- an observation in line with our reasoning. van citters et al. (34), observed that upon blebbistatin intervention in epithelial cells, the MSD from two-point microrheology was largely unaffected - a result that is in contrast with our single-particle tracking observations and needs to be resolved.

With nocodazole and taxol treatments the dynamics of EGs slows down, but to a different degree. Immunofluorescence staining showed that the actin network was largely intact under the influence of these drugs, suggesting that they interfere with the microtubule polymerization forces (i.e. the active driving force, f) to a different extent.

\subsection{Conclusions}

Our results demonstrate that endogenous granules exhibit universal diffusive dynamics that is most likely driven by the microtubule bending dynamics due to active polymerization forces. We are led to this remarkable conclusion by the following key points of evidence (i) actin network disruption (by latrunculin) does not significantly affect the dynamics of EGs (ii) EG dynamics is quite sensitive to drugs (nocodazole and taxol) affecting microtubule network (iii) The dynamics of EGs under presumably passive conditions (temperature reduction to 
$24^{\circ} \mathrm{C}$ ) is well described by a semiflexible polymer model that assumes EGs linked to microtubule network (iv) The magnitude of displacement exhibited by EGs in untreated cells is consistent with microtubule bending fluctuations driven by a motor-driven force.

In addition our findings lead to the following surprising implications. First, endogenous granules could be used as non-invasive microrheological probes for microtubule network dynamics in living cells. Second, literature studies that have been using endogenous granules to probe intracellular rheology might in fact be probing the mechanical properties of primarily the microtubule network rather than the entire living cytoskeleton. This could partly explain the wide variation in cell modulus reported by MPTM and other techniques such as atomic force microscopy and magnetic twisting cytometry (16).

\section{Acknowledgements}

We are grateful to D. Wirtz for advice on ballistic particle injection, V. Breedveld for the particle tracking code. We thank Andries van der Meer and Andre Poot for providing cell lines and cell culture training. Thanks Moumita Das for illuminating discussions on microtubule buckling. This research was supported by the Cell Stress program of the MESA+ Institute of Nanoechnology.

\section{References}

1. Kamm, R. D., Mofrad, M. R. K. (2006) Cytoskeletal mechanics: models and measurements (Cambridge University Press, New York).

2. Janmey, P. A., Weitz, D. A. (2004) Trends Biochem. Sci. 29, 365-370.

3. Heidemann, S. R., Wirtz, D. (2004) Trends Cell Biol. 14, 160-166.

4. Pollard, T. D. (2003) Nature 422, 741-745.

5. Balland, M., Richert, A., Gallet, F. (2004) Eur. Biophys. J. 34, 255-261.

6. Bursac, P., Lenormand, G., Fabry, B., Oliver, M., Weitz, D. A., Viasnoff, V., Butler, J. P., Fredberg, J. J. (2005) Nat. Mater. 4, 557-561.

7. Alcaraz, J., Buscemi, L., Grabulosa, M., Trepat, X., Fabry, B., Farre, R., Navajas, D. (2003) Biophys. J. 84, 2071-2079.

8. Weihs, D., Mason, T. G., Teitell, M. A. (2006) Biophys. J. 91, 4296-4305.

9. Desprat, N., Richert, A., Simeon, J., Asnacios, A. (2005) Biophys. J. 88, 2224-2233.

10. Tseng, Y., Kole, T. P., Wirtz, D. (2002) Biophys. J. 83, 3162-3176.

11. Lau, A., Hoffman, B. D., Davies, A., Crocker, J. C., Lubensky, T. C. (2003) Phys. Rev. Lett. 91, 198101. 
12. Yamada, S., Wirtz, D., Kuo, S. C. (2000) Biophys. J. 78, 1736-1747.

13. Yanai, M., Butler, J. P., Suzuki, T., Sasaki, H., Higuchi, H. (2004) Am. J. Physiol. Cell Physiol. 287, C603-C611.

14. Tolic-Norrelykke, I. M., Munteanu, E. L., Thon, G., Oddershede, L., Berg-Sorensen, K. (2004) Phys. Rev. Lett. 93, 078102.

15. Dangaria, J. H., Butler, P. J. (2007) Am. J. Physiol. Cell Physiol. 293, 1568-1575.

16. Hoffman, B. D., Massiera, G., van Citters, K. M., Crocker, J. C. (2006) Proc. Natl. Acad. Sci. USA. 103, 10259-10264.

17. Rogers, S. S., Waigh, T. A., Lu, J. R. (2008) Biophys. J. 94, 3313-3322.

18. Panorchan, P., Lee, J. S., Daniels, B. R., Kole, T. P., Tseng, Y., Wirtz, D. (2006) Meth. Cell. Biol. 83, 115-140.

19. Crocker, J. C., Grier, D. G. (1996) J. Colloid Int. Sci. 179, 298-310.

20. Caspi, A., Granek, R., Elbaum, M. (2000) Phys. Rev. Lett. 85, 5655-5658.

21. Pangarkar, C., Dinh, A. T., Mitragotri, S. (2005) Phys. Rev. Lett. 95, 158101.

22. Snider, J., Lin, F., Zahedi, N., Rodionov, V., Yu, C. C., Gross, S. P. (2004) Proc. Natl. Acad. Sci. USA. 101, 13204-13209.

23. Duits, M. H. G., Li, Y., Vanapalli, S. A., Mugele, F. (2009) Phys. Rev. E, 79, 051910.

24. Brangwynne, C. P., Koenderink, G. H., MacKintosh, F. C., Weitz, D. A. (2008) Phys. Rev. Lett. 100, 118104.

25. Brangwynne, C. P., MacKintosh, F. C., Weitz, D. A. (2007) Proc. Natl. Acad. Sci. USA. 104, 16128-16133.

26. Gittes, F., MacKintosh, F. C. (1998) Phys. Rev. E. 58, R1241-1244.

27. Caspi, A., Elbaum, M., Granek, R., Lachish, A., Zbaida, D. (1998) Phys. Rev. Lett. 80, 1106-1109.

28. Gittes, F., Mickey, B., Nettleton, J., Howard, J. (1993) J. Cell. Biol. 120, 923-934.

29. Brangwynne, C. P., MacKintosh, F. C., Kumar, S., Geisse, N. A., Talbot, J., Mahadevan, L., Parker, K. K., Ingber, D. E., Weitz, D. A. (2006) J. Cell. Biol. 173, 733-741.

30. Li, T. (2008) J. Biomech. 41, 1722-1729.

31. Das, M., Levine, A. J., MacKintosh, F. C. (2008) Eur. Phys. Lett. 84, 18003.

32. Fabry, B., Maksym, G. N., Bulter, J. P., Glogauer, M., Navajas, D., Fredberg, J. J. (2001) Phys. Rev. Lett. 87, 148102.

33. Howard, J., Hyman, A. A. (2003) Nature. 422, 753-758.

34. van Citters, K. M., Hoffman, B. D., Massiera, G., Crocker, J. C. (2006) Biophys. J. 91, 3946-3956. 


\title{
Chapter 5
}

\section{Dynamics of Ballistically Injected Latex Particles in Living Human Endothelial Cells}

\begin{abstract}
We studied the dynamics of ballistically injected latex particles (BIP) inside endothelial cells, using video particle tracking to measure the mean squared displacement (MSD) as a function of lag time. The MSD shows a plateau at short times and a linear behavior at longer times, indicating that the BIP are trapped into a viscoelastic network. To reveal more about the molecular constituents and the dynamics of this actin network, we added a variety of drugs. Latrunculin and Jasplakinolide aimed at intervening with the actin network caused a strong increase in MSD, whereas Taxol aimed at microtubules gave no measurable change in MSD. Additional corroborating information about these drug effects were obtained from MSD amplitude and exponent distributions and from fluorescent staining images of the actin and microtubule networks. Our evidence strongly suggests that BIP are primarily embedded in the actin network. Additional drug interventions aimed at disabling non-thermal forces could not conclusively resolve the nature of the forces driving BIP dynamics.
\end{abstract}

This chapter has been published in Biorheology 46(4), 309-321 (2009). 


\subsection{Introduction}

The use of video microscopy to study the statistical motions of colloidal particles inside living cells (intracellular particle tracking), has potential to become one of the future methods for diagnosis of individual cells. There are several reasons for this: 1) the dynamics of intracellular particles is sensitive to the mechanical state of a cell, which in turn depends on its health condition $[13,15,20]$; 2) colloidal particles are easily visualized, and their size is ideally suited to probe the cytoskeleton's mechanics [35]; 3) since low power light sources can be used, the measurements are in principle non-invasive; 4) because many intracellular particles are available [6,32] or can be introduced [27], and representative responses can be obtained for individual cells, and 5) combination of microscopy and microfluidics should allow for parallel or high throughput analysis of cells in chips [9,26]. However the complex architecture and dynamics of the living cell also creates a major obstacle: without deeper insight into the origins of the intracellular particle motions, only an empirical knowledge is generated about the relation between the measured mean squared displacements (MSD) of the particles and the state of the cell, to cite one example. While this can be valuable for distinguishing between healthy and diseased cells, it contributes little to achieving generic insights. In contrast, if intracellular particle dynamics could be linked more systematically to physical quantities (such as rheological properties) or the behavior of specific cytoskeletal structures (such as the dynamics of the actin network), then a more conceptual picture would be obtained and a comparison between different measurements might be possible. For externally enforced global deformations of the cell, successes were recently achieved through the use of either AFM [7] or optical stretching [11] to measure the apparent elastic modulus of the cell. Intracellular particle tracking appears to have a similar potential $[28,33]$.

Developments in the field thus call for fundamental studies into the origins of erratic intracellular particle motions. From the literature [13,19,30,31,34], it becomes clear that a measured MSD depends on: 1a) in what kind of micro environment (ME) the particles inhabit; 1b) how the particles interact with this $\mathrm{ME}$ and 1c) what drives the motions of the particles. These three questions are obviously related to each other, and often the answers are at least partially unknown.

The ability to address any of these sub-questions can already be valuable. For example, the annihilation of nonthermal (ATP dependent) driving forces through chemical intervention could allow use of the fluctuation-dissipation theorem as 
done in microrheology [23]. In that case, intracellular rheological properties such as the visco-elasticity of the cytoskeleton could be obtained. Encouraging results along this line were obtained by Hoffman et al. [13] where a universality in particle dynamics was found after ATP depletion. Also, knowledge of the physico-chemical interactions between a particle and its ME could simplify interpretation. For example, probes with a binding affinity for specific intracellular components (such as microtubules or the actin network) could be used as sensors for the fluctuations of these networks. Paradoxically, achieving such simplifying conditions requires insight into the complexity of the cell. Additional questions which then arise are: 2) can probes with a similar surface chemistry be assumed to occur in comparable micro environments? and 3) can non-thermal driving forces indeed be eliminated, or alternatively, revealed?

This paper reports on an attempt to address questions 1-3. We present experiments on human endothelial cells, for which we found a rather different dynamics for Endogenous Granules (EG), as described in another paper [32]) than for microinjected latex particles in the present paper. Our carboxylated poly(styrene) latex particles have also been studied in previous particle tracking and microrheology studies, because of their negative surface charge and low binding affinity to actin [24,30,31]. Moreover, there is no evidence that these particles are 'recognized' by the cell and hence undergo important changes (like encapsulation) at relevant experimental timescales. However, in what local ME these particles occur has not been systematically studied, and whether or not motions of these particles in these cells are driven by ATP-dependent processes or not (at typical timescales for video particle tracking), has not been demonstrated either.

To reveal the microenvironment of the BIP (question 1), we exposed our cells to a variety of drugs that specifically interfere with either the actin network or microtubules. We also attempted to shed more light on the role of ATPdependent processes, by depleting the intracellular ATP and by adding Blebbistatin, whose primary effect is to disable actomyosin contractility. Besides MSD averaged within cells (and over different cells), we also studied variations in the dynamics displayed by different BIP in the same cell. This allowed us to also address the questions 2 and 3, through study of histograms for the MSD amplitude and exponent.

This paper is organized as follows: in section 5.2 we will discuss all experimental aspects of cell culture, drug treatments, staining, ballistic particle injection, microscopy and particle tracking. The analysis of mean squared 
displacements is discussed in section 5.3. In section 5.4 we present the effect of drugs on ensemble averaged MSD, on the distributions of the individual MSD and on cytoskeletal structure as visualized by staining. In section 5.5, conclusions are drawn.

\subsection{Materials and methods}

\subsubsection{Cell Culture}

Human microvascular endothelial cells (Hmec-1) at 25-30 passages, were cultured at $37^{\circ} \mathrm{C}$ in a humidified $5 \% \mathrm{CO}_{2}$ environment in endothelial cell growth medium containing hydrocortisone, hFGF, R3-IGF-1, ascorbic acid, hEGF, gentamicin, heparin, and $2 \%$ fetal bovine serum (EGM-2, Lonza, Basel, Switzerland). For our microscope experiments, cells were plated on a Delta $\mathrm{T}$ culture dish (Bioptechs, Butler, PA, USA), that had been coated for 1 hour with fibronectin $(100 \mu \mathrm{g} / \mathrm{mL}$ solution). A heated lid was used to prevent solvent evaporation losses, and cells were maintained at $37^{\circ} \mathrm{C}$ and $5 \% \mathrm{CO}_{2}$ during tracking experiments. Cultures were typically at $50 \%$ confluence, which allowed studying individual cells. Under these conditions, and before drug treatment, most of our cells showed morphologies such as those illustrated in Fig. 5.1A. Also different morphologies (e.g. cells undergoing division) were sometimes encountered. These cells were excluded from the analysis, to minimize contamination of the results.

\subsubsection{Probe Types}

In this study we mainly worked with ballistically injected particles (BIP). For these, we chose monodisperse, red-fluorescent carboxylated poly(styrene) latex particles with diameter $0.2 \mu \mathrm{m}$ (Invitrogen) and excitation/emission wavelength $565 / 610 \mathrm{~nm}$. These probes appear as bright spots in confocal fluorescent mode (Fig. 5.1A). For comparative purposes, we also studied some cells with endogenous granules (EG). These granules, which appear as dark objects under phase contrast microscopy (Fig. 5.1A), were confirmed to be mainly lipid droplets after staining with Nile Red (Invitrogen). The mean size of the granules was assessed to be $\approx 0.5 \mu \mathrm{m}$. Unless mentioned otherwise, BIP and EG were studied in separate cells.

\subsubsection{Ballistic injection}

Ballistic injections of tracer particles were carried out using a Biolistic gun (Biorad) and a procedure similar to that described in Panorchan et al. [21,27] (but without hepta adapter). Using optimized injection parameters (vacuum level 28 $\mathrm{mm} \mathrm{Hg}$, Helium pressure $1350 \mathrm{psi}$, carrier disk-to-sample distance $3 \mathrm{~cm}$ ), we 
achieved a $\sim 50 \%$ survival rate of the cells. After bombardment, a recovery time of $30 \mathrm{~min}$ was allowed before trypsinizing and replating the cells. Of the surviving cells, only those that contained 15-40 particles were selected for tracking experiments. Sometimes aggregates of BIP were observed; these were excluded from the analysis.

\subsubsection{Drug interventions on cytoskeleton and myosin motors}

All drugs were purchased from Sigma Aldrich, except Blebbistatin (Tebu-bio, Belgium). The following interventions were done: Latrunculin A (200 nM, incubation time $2 \mathrm{hrs})$, Jasplakinolide $(25 \mu \mathrm{M}, 1 \mathrm{hr})$, Nocodazole $(5 \mu \mathrm{M}, 3 \mathrm{hrs})$, Cephalomannine (Taxol, $100 \mu \mathrm{M}, 1 \mathrm{hr}$ ), Blebbistatin $(50 \mu \mathrm{M}, 2 \mathrm{hrs})$. 2-Ddeoxyglucose and Sodium Azide at final concentrations of $50 \mathrm{mM}$ and $0.05 \%$ respectively were applied for $30 \mathrm{~min}$ as an ATP-depletion cocktail. Each drug intervention effect was measured by $2-5$ independent experiments, i.e. adding freshly prepared drug solution to fresh cells. A typical tracking experiment lasted 2-3 hours after the end of drug incubation.

\subsubsection{Immunofluorescence microscopy}

To stain the intracellular structures, cells were rinsed with phosphate buffer saline (PBS) solution and fixed with $4 \%$ formaldehyde in PBS at $37^{\circ} \mathrm{C}$ for 8 min. Next, cells were permeabilized with a PBS solution containing $0.2 \%$ Triton-X and $0.1 \%$ BSA (Sigma-Aldrich). FITC-phalloidin $(1 \mu \mathrm{M})$ was used to stain Factin. For microtubule staining, mouse anti- $\alpha$-tubulin antibody $(1 \mathrm{mg} / \mathrm{L})$ was applied for 20 minutes, after which the sample was thoroughly washed with PBS and further immersed in a solution of Rhodamine conjugated goat anti-mouse antibody $(1 \mathrm{mg} / \mathrm{L})$ for 20 minutes. Immuno-fluorescence was viewed using our confocal microscope described below. Stainings were performed immediately after the incubation time.

\subsubsection{Multiple particle tracking}

Probe particles were visualized using the UltraView LCI10 system (Perkin Elmer), in which a Yokogawa spinning-disk confocal unit is combined with a Nikon Eclipse TE-300 inverted microscope. A 100× (NA 1.3) oil immersion objective was used. BIP were visualized in confocal fluorescence mode (using the $564 \mathrm{~nm} \mathrm{Kr}$ line), while endogenous granules were imaged in phase contrast mode. Approximately 2500 images were recorded with a (Hamamatsu) 12-bit CCD camera at $\sim 17$ frames per second for a typical duration of 150 seconds. The spatial resolution corresponding to the images was $0.13 \mu \mathrm{m}$ per pixel. In each cell, 50-100 EG and 15-40 BIP were tracked. The particles were identified using the publicly available particle-tracking code [14] based on ref. [5], written and 
extended in Interactive Data Language (IDL) by several authors. The localization error was measured to be $\approx 15 \mathrm{~nm}$ (Fig. 5.1B) by measuring the MSD of probe particles attached to a coverslip. For each experimental condition (i.e. drug treatment), 10 different cells were studied, giving $10^{3}-5 \times 10^{3}$ trajectories (larger than the total number of particles, due to repeated displacements in and out of the focal plane).

\subsubsection{Data Analysis}

Our data analysis, more extensively described in [8] is based on the particle tracking rheology method as demonstrated by Gittes et al. and Mason et al. [10,22] and first applied to living cells in 2002 by Tseng et al. [30]. Besides the usual Mean Squared Displacement $\left\langle\Delta r^{2}\right\rangle(\tau)$, obtained by averaging over displacements of the same particle as well as over the different particles present in the cell, we also examined individual particle MSDs (iMSDs). Both MSDs and iMSDs were analyzed by fitting a power law to their local behavior around a chosen lagtime $\tau_{0}$ :

$$
\left\langle\Delta r^{2}\right\rangle \approx A\left(\tau / t_{0}\right)^{\alpha}
$$

where $A$ is the amplitude, $\tau$ the lagtime and $t_{0}$ the unit exposure time $(60 \mathrm{~ms}) . A$ and $\alpha$ provide complementary information on the respective displacement magnitude and the type of the motion (from sub to super-diffusive). The iMSD were analyzed at different lagtimes $\tau_{0}$, producing an amplitude $A\left(\tau_{0}\right)$ and exponent $\alpha\left(\tau_{0}\right)$. Histograms for these quantities were obtained by averaging over the different cells under the same experimental condition (i.e. drug treatment). These distributions are compared with reference curves for a viscous liquid and an elastic solid in Sec. 5.4. 


\subsection{Results}

\subsubsection{Ensemble dynamics of BIP}
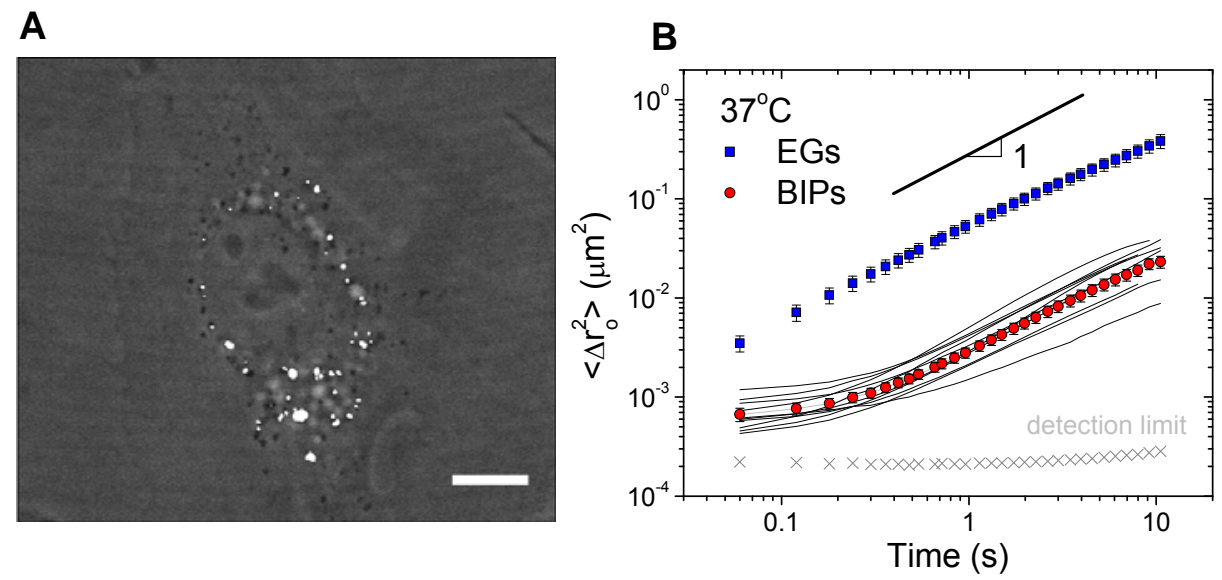

Fig. 5.1: (A) Illustration of the cellular distributions of BIP (bright spots) and EG (dark spots)in a single Hmec-1 cell. Scale bar is $10 \mathrm{~mm}$. (B) Mean Square Displacement versus lagtime functions for EG (squares) and BIP (circles) in cells under physiological conditions. Results are averaged over 10 cells, and error bars indicate the standard deviation of the mean. For BIP also the results for the individual cells are shown. Cross symbols show a measurement of the (BIP) latex particles glued onto a coverslip.

As a reference case we first consider the cells without interventions (other than the injection with BIP). The typical morphology of a single Hmec-1 cell is shown in Fig. 5.1A, which also illustrates how the BIP are spread over the intracellular space. Fig. 5.1B shows the MSD obtained by averaging over 10 different Hmec-1 cells, along with the results for the individual cells. As will be shown, these differences between cells are modest compared to the effects caused by the interventions. Two characteristic regions can be found in the MSD-time curve: (i) for short lag times $(<0.3 \mathrm{~s})$, the onset of a plateau is found, indicative of an elastic environment, and (ii) for $\tau>1.0 \mathrm{~s}$ a nearly diffusive behavior is found, evidenced by a powerlaw exponent $\alpha$ of $0.92 \pm 0.02$.

At this point it is also interesting to compare the dynamics of BIP to that of the endogenous granules studied in the same cells earlier by us [32]. The MSD taken from the latter study are included in Fig. 5.1B. Remarkably, for EG the amplitude is almost an order of magnitude higher than for BIP, although the (typical) radius of the EG is 2.5 times larger. In addition, the EG do not display a 
quasi-plateau region in the MSD at short times, but an apparently diffusive behavior for the entire time range.

To corroborate this result, we performed an additional experiment in which both probes were tracked inside the very same cell. Here, the plot of all individual MSD was found to correspond well to combined plots of iMSD from EG and BIP measured in separate cells (not shown). In addition we verified that the different dynamics of the two probes is not due to obvious differences in intracellular location. Making a distinction between perinuclear and perimembrane areas for 10 different Hmec- 1 cells, we found that $91 \%$ of the BIP were found in the perinuclear area, while it was $86 \%$ for the EG. These observations confirm the fact that the strong (qualitative and quantitative) differences in dynamics between EG and BIP in Hmec-1 cells are due to the occurrence of these probes in entirely different local microenvironments.

\subsubsection{Interpretative approach}

As also pointed out in other particle tracking studies in cells (e.g. [34]), the complexity of the analysis of intracellular MSD necessitates a certain interpretative approach. MSD measurements cannot provide specific clues unless combined with additional information. A priori, our MSD data could reflect mechanical properties of the actin network (AN), microtubules (MT), or the cytoskeleton as a whole. Also a priori, thermal collisions or ATP driven processes or a combination thereof could be responsible for the mechanical excitations (especially in the time range of $0.1-30 \mathrm{~s}$ as addressed by our experiments, see [25]). Hence we will discuss differences or changes in MSD magnitude only in the context of a competition between driving forces (tending to increase the amplitude) and resistance (to be called "stiffness") offered by the visco-elastic network (tending to reduce the amplitude).

To explore changes in driving force or stiffness, we added a variety of drugs aimed at selectively interfering with either the actin network or the microtubules. Since interpretation can be confounded by secondary effects (responses by the cell) we explored two different drugs per cytoskeleton component. Also drugs aimed at eliminating ATP-dependent driving mechanisms were explored. Images of cells stained for actin and tubulin were assumed to qualitatively represent cytoskeletal changes.

\subsubsection{Drug interventions aimed at the actin network}




\subsubsection{Latrunculin A}

In an attempt to break down the F-actin network, Latrunculin A (LA) was applied at a concentration of $0.2 \mu \mathrm{M}$ during 2 hours (similar conditions as in [13]) before the particle tracking experiments were started. The averaged MSD (top curve in Fig. 5.2) shows that the elastic plateau for $\tau<0.3 \mathrm{~s}$ has largely disappeared, and that the slope of the MSD curve has become close to 1.0 over a large time range. An increase in MSD might be expected when the stiffness of the particle's micro environment is reduced while the driving forces are kept intact. The occurrence of a power law exponent of 1.0 over two decades could suggest that the viscoelastic cytoplasm had transformed into a viscous liquid. This issue will be further addressed in Section 5.3.5.

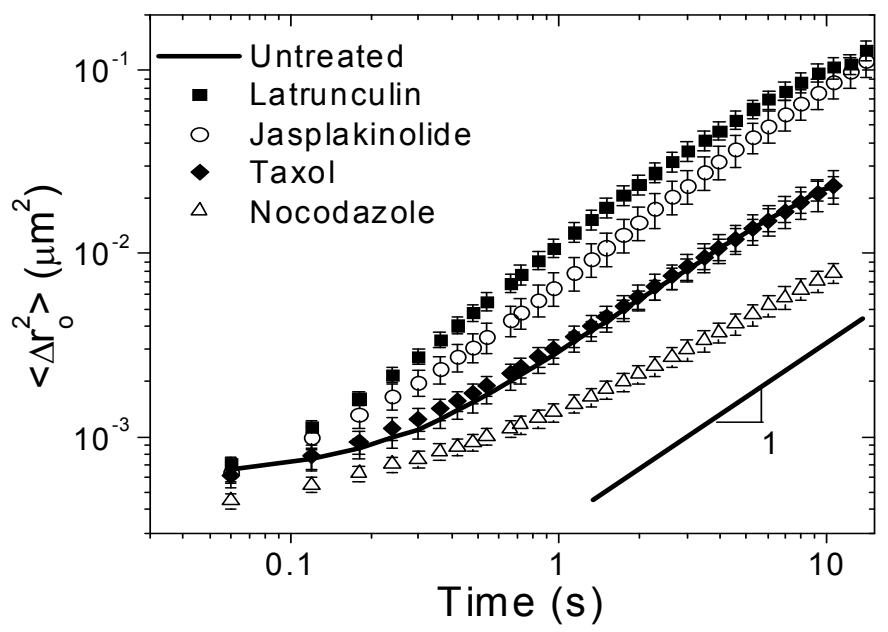

Fig. 5.2: Effects of various cytoskeletal interventions on BIP dynamics. MSD of untreated BIP (-), after exposure to latrunculin $A(\mathbf{\square})$, jasplakinolide(O), taxol $(\downarrow)$, nocodazole ( $\Delta)$.

The disappearance of the elastic region at $\tau<0.3 \mathrm{~s}$ in LA-treated cells turned out to be highly reproducible. Shorter exposures to LA were also explored, where the effect on MSD was less pronounced (data not shown). As a control, we also performed a cell recovery test by replacing LA treated culture medium with the normal culture medium. Two hours after the LA had been removed, a significant number of the cells exhibited MSD in which the elastic plateau had been fully restored. This suggests that the intervention with LA is reversible. More importantly, it shows that the change in the BIP dynamics was not related to a 
change in cell status (for example, apoptosis) but due to the depletion of the actin network.

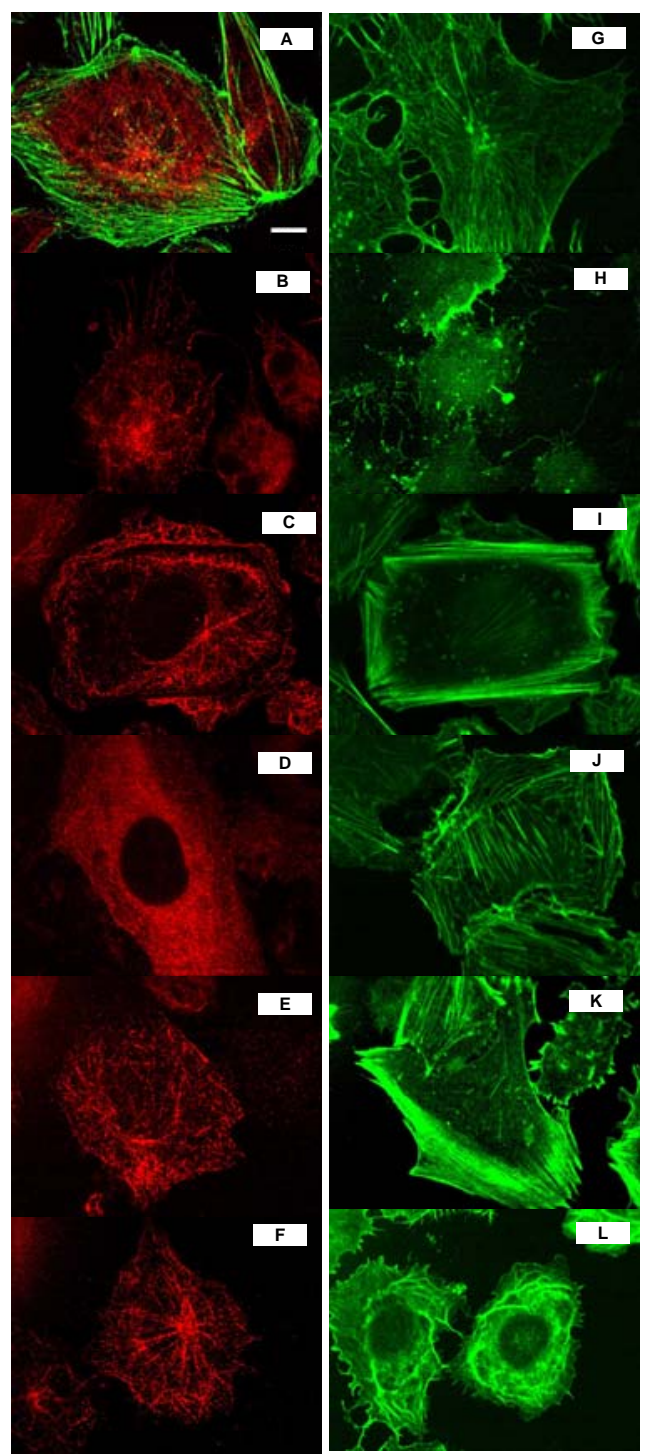

Fig. 5.3: Fluorescent staining images of actin (green) and microtubules (red) in Hmec-1 cells before (A) \& after treatments with drugs (B-L). G: ATP depletion (actin only). B,H-latrunculin A; C, I-jasplakinolide; D,J-nocodazole; E, Ktaxol; F,L-blebbistatin. Scale bar is $10 \mu \mathrm{m}$.

The effect on LA on the actin cytoskeleton was corroborated by the microscopy images. In line with the observations in [34], the cell morphology was found to 
have changed into a typical 'balled-up' shape. Our fluorescent staining images showed that the stress fibers had completely disappeared. Only discrete actin aggregates at the peri-membrane area were still visible (see Fig. $5.3 \mathrm{H}$ ).

\subsubsection{Jasplakinolide}

Jasplakinolide is known for its specific interference with the (de-) polymerization of actin, and has also been used in studies of the actin cytoskeleton. However its stabilizing effect on the actin network in vitro [3] does not necessarily transfer to a similar effect in vivo, as evidenced by a study in which Jasplakinolide was found to disrupt the actin network inside cells [4]. After exposing our Hmec-1 cells to $25 \mu \mathrm{M}$ Jasplakinolide for $1 \mathrm{~h}$ followed by fluorescent staining, we found that the actin appeared depleted in the perinuclear area, but also that actin stress fibers still persisted in the peri-membrane area (see Figs. 5.3C and 5.3I). Interestingly, this suggests that mechanical methods that mostly probe the cell membrane, such as Magnetic Twisting Cytometry or (low amplitude) Atomic Force Microscopy, could measure a different effect of Jasplakinolide than the intracellular techniques such as our PTR method.

In our experiment, the depletion of actin in the perinuclear area (where most of our particles are found) again caused an increase in the MSD amplitude and powerlaw exponent. This is in line with expectations based on the LA intervention experiment. Apparently the effect of Jasplakinolide is slightly less strong compared to that of LA.

\subsubsection{Drug interventions aimed at microtubules}

\subsubsection{Taxol}

Taxol has been widely explored for its ability to stabilize MT [29,37]. It inhibits the depolymerization of MT and hence changes the balance between MT growth and catastrophe. The consequence is that many short MT units are formed. Indeed, our fluorescent staining images (Fig. 5.3, E, K), revealed that after treatment with $100 \mu \mathrm{M}$ for $1 \mathrm{~h}$, the majority of MT had become fragmented (or highly curved). In contrast, no changes in the actin network were visible. The effect of taxol intervention on the averaged MSD of BIP turned out to be negligible, which is remarkable for such a drastic intervention. The simplest explanation for this is that, neither the driving force nor the stiffness of the micro-environment for the BIP is changed. Additional support for this argument will be provided in $\operatorname{Sec}$ 5.3.5. 


\subsubsection{Nocodazole}

Nocodazole interferes in a rather different way with MT than does taxol. It can bind to the $\beta$-subunit of tubulin and promote depolymerization of MT. As a result, the MT are destroyed, which can also lead to changes in the cell shape. On longer time scales $(>12 \mathrm{hrs})$ Nocodazole interferes with the cell cycle, synchronizing cells to the G2/M state. To minimize this effect, we used a high dosage $(5 \mu \mathrm{M})$ of Nocodazole during a short time (3 hours). As claimed in [36], this treatment should not increase cell death or change the actin or intermediate filament cytoarchitecture. The fluorescent staining images in Fig. 5.3, D and J confirm the expected disappearance of the MT network, whereas the actin network appears to be unaffected. The amplitude and power-law exponent of the MSD show a significant reduction due to Nocodazole treatment, implying a reduction in driving force and/or a stiffening of the micro-environment of the BIP.

\subsubsection{Individual dynamics of BIP}
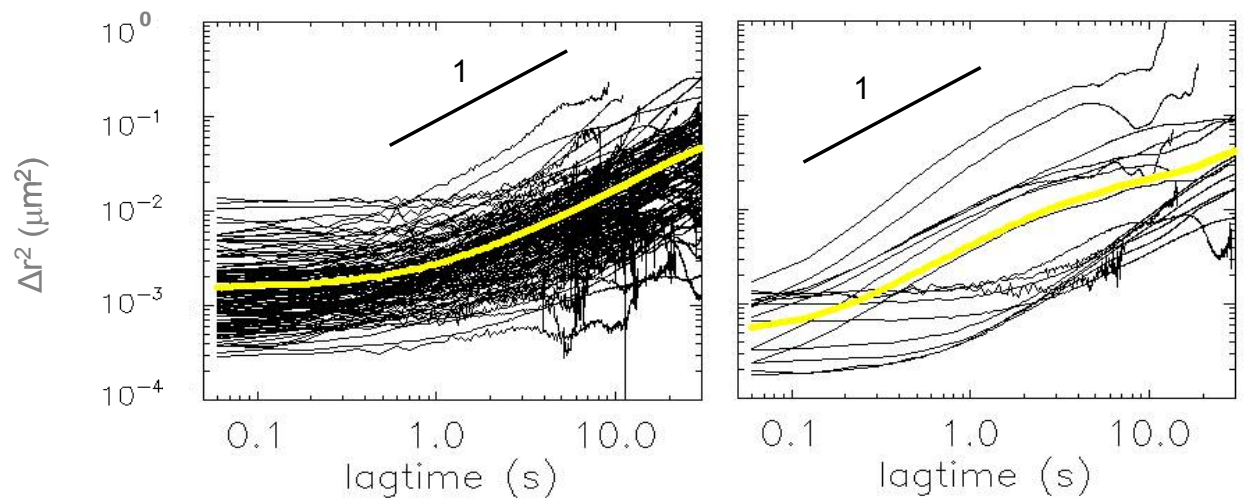

Fig. 5.4: Overview of individual MSD (black) and total MSD (light grey) for two selected Hmec-1 cells loaded with BIP. Fig 5.4a shows the transition from a plateau to a diffusive behavior in the overall MSD. Fig. 5.4b shows how both elastic-like and diffusive-like behaviors can occur at small lagtimes in the same cell.

To further study the effects of the drug interventions, we now turn to the analysis of (the statistics of) individual trajectories. First we consider Figs. 5.4a and b, which show typical trajectories for BIP inside two different Hmec-1 cells. As with other cells [30], here it is also observed that a considerable spread exists amongst the trajectories of different particles inside the same cell. 
We analyzed this heterogeneity by fitting local power laws (Sec. 5.2.7) to individual MSD functions and then grouping the results for different cells at the same condition into the same histogram. To allow objective comparison between different experiments [8] we segmented all trajectories into blocks of 200 steps before calculating the iMSDs. Fitting the local amplitude (A) and exponent $(\alpha)$ around $\tau \approx 0.1 \mathrm{~s}$, produces the histograms in Figs. 5.5a and b. Fitting around $\tau \approx$ $1.0 \mathrm{~s}$ produces Fig. $5.5 \mathrm{c}$.
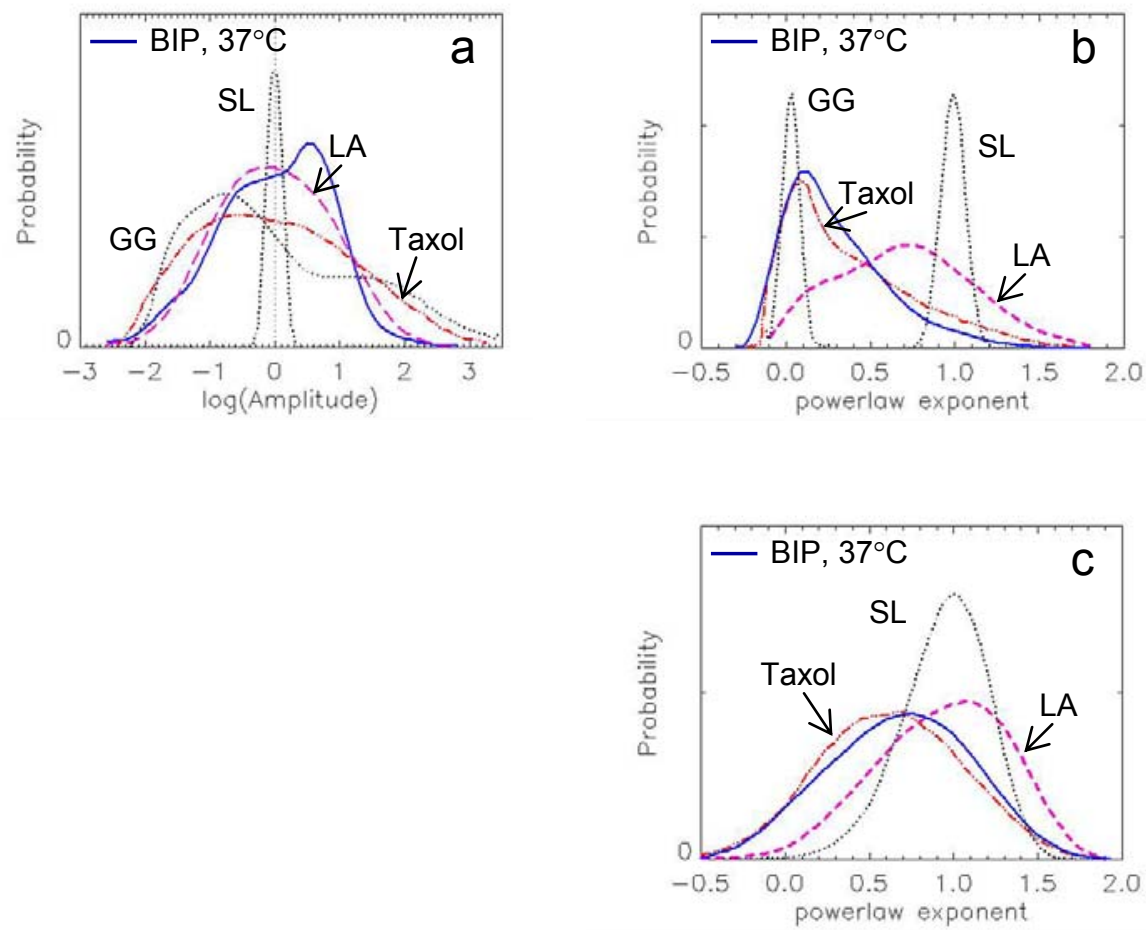

Fig. 5.5: Probability distributions for the (normalized) amplitude of the iMSD at $\approx 0.1 \mathrm{~s}(a)$, and its local exponent at $\approx 0.1 \mathrm{~s}$ (b) and $\approx 1.0 \mathrm{~s}$ (c). In a) all amplitudes have been divided by the average over 10 cells at the same condition. Solid lines: BIP in Hmec-1 cells at $37^{\circ} \mathrm{C}$. Dashed-dotted curves: after Taxol treatment. Dashed curves: after treatment with Latrunculin A (LA). For comparison, also reference curves for particles in a simple liquid (SL) and a gelatin gel (GG) are given. In a) and b) these have been multiplied with 0.2 and 0.4 respectively.

First we remark that all curves in Fig. 5.5 show broadening due to the finite number (i.e. 200) of steps per (segmented) trajectory. This is clearly illustrated by the dotted curves marked with SL (simple liquid), which represent a 
measurement of particles with radius $\mathrm{a}=100 \mathrm{~nm}$ in a water/glycerol mixture with viscosity $\eta \approx 100 \mathrm{mPas}$ at $\mathrm{T}=298 \mathrm{~K}$. In such a liquid, all particles have the same expectation value for the amplitude $(\mathrm{A}=2 \tau \mathrm{kT} / 3 \pi \eta \mathrm{a})$ and exponent $(\alpha=1.0)$. However, due to the limited statistics, a measurement inaccuracy is superimposed [8]. To illustrate this further, we also present a distribution of $\alpha$ measured for an elastic gelatin gel $(\mathrm{GG})\left(10 \mathrm{wt} \%\right.$ in water at $\left.24^{\circ} \mathrm{C}\right)$ in Fig. $5.5 \mathrm{~b}$. Since this curve is centered around $\alpha \approx 0$, the statistical broadening (or measurement error) now gives rise to negative values. Also the occurrence of negative exponents down to $\approx-0.15$ for the BIP in Fig. 5.5 b should be seen in this light.

In spite of the broadening, several interesting observations can still be made from Fig. 5.5. First of all it is clear that BIP under physiological conditions do not display a simple behavior. At lagtimes $\tau<0.3 \mathrm{~s}$ where a plateau-like MSD was found, both the amplitude and the exponent display a broad distribution. The tail in Fig. 5.5b is at least partly due to a few diffusive trajectories, as are illustrated in Fig 5.4b. We recall that all histograms in Fig. 5.5 are averages over multiple (typically 10) cells. Making up the histograms for individual cells, the distributions were found slightly narrower but still considerably broader than the SL distribution (data not shown).

Secondly we discuss the effect of administering LA and Taxol on the histograms for BIP in Fig. 5.5. These two interventions are especially interesting in view of their dramatically different effects on the average MSD of EG [8] and BIP (Fig. 5.2). For BIP, LA was found to give an average MSD that suggested simple diffusive behavior. But as seen from Fig. 5.5, it results in fact in a more complex behavior. The distribution for $\alpha$ now reveals superdiffusive contributions (both at 0.1 and at $1.0 \mathrm{~s}$ ), since the fraction with $\alpha>1.0$ is clearly larger than that of the SL curve. This suggests that at least after the treatment with LA, non thermal driving forces are acting on BIP. Possibly, these forces also acted in the absence of LA (but then without manifesting themselves via the distribution of $\alpha$ ). In contrast, exposing the cells to Taxol has an almost negligible effect on the exponent distribution. This indicates that it is rather unlikely that the active motions of MT are driving the dynamics of BIP (even if this happened indirectly e.g. as with MT bending fluctuations shaking the actin network [2]).

Finally we note from Fig. 5.5a that the amplitude distribution is broad for all systems except SL. The curve for LA once more corroborates that the dynamics after intervention with LA is not simply diffusive. This could suggest that there is still an intact network that is capable of modulating the motions of the BIP. 
Interestingly, even the curve for gelatin (rheologically speaking, a simple elastic solid) shows a large amplitude heterogeneity (we have no tentative explanation for the detailed shape of this distribution). The amplitude heterogeneity for both the cytoskeleton and gelatin gels could point to a distribution in local stiffness, e.g. due to variations in the density of elastic strands. Alternatively it could also indicate a heterogeneity in probe-matrix contacts, as previously suggested [34]. This is an interesting issue, which merits further study.

\subsubsection{Drug interventions aimed at disabling active processes}

\subsubsection{ATP depletion}

Inspired by the results of [13] we also attempted to switch off the non-thermal driving forces by depleting ATP. Unlike previous studies with EG [2,32] in which strong amplitude reductions were observed, we found a twofold increase in the MSD-time curve for our BIP, without any change in the shape of the curve. Also the histograms for $A$ and $\alpha$ did not significantly change due to the ATP depletion (results not shown). This counterintuitive increase in average MSD implies that there were secondary effects. We attribute these to a decrease in the stiffness, possibly caused by a weakening of the actin network. A partial depletion of this network is suggested by the images of the stained cells (Fig. $5.3 \mathrm{G})$.

\subsubsection{Blebbistatin}

Since this drug specifically inhibits the action of myosin II isoforms, by preventing reattachment of the myosin head after the power stroke [18], its effect could provide a clue as to the importance of actomyosin contractility. At the level of MSD we found that the amplitude was reduced by a factor 2, while the exponent $\alpha$ at $\tau \approx 1 \mathrm{~s}$ had decreased to $0.7+/-0.1$ (not shown). However, we also found that the morphology of the cells had changed (see Figs. 5.3F and 5.3L): the cells had shrunk, peripheral stress fibers had been lost, and accumulation of clumps of actin had appeared in cytoplasm. This indicates that not only the primary effect on the myosin motors but also secondary effects like a stiffening of the cytoskeleton may have contributed to the change in MSD.

\subsection{Discussion}

\subsubsection{Micro-environment of the BIP in the living cytoskeleton}


Most of our observations give positive indications that our injected carboxylated latex particles are embedded in the actin network. The interventions with Latrunculin and Jasplakinolide provide strong supporting evidence - breaking down the AN (in the perinuclear region where almost all particles reside) gives rise to a disappearance of the elastic plateau, and increased motion amplitudes. The shape of the MSD (time) curves for the BIP can then be explained from the transient nature of the AN. Since our BIP are bigger than the reported mesh size for intracellular F-actin (50 $\mathrm{nm}$ in ref [30], $100 \mathrm{~nm}$ in ref [12]), they should become trapped in the cages provided by the actin network. However since the AN is a dynamic entity (modulated by a number of proteins that crosslink, bundle, sever and cap actin filaments [1]) the cages will have a certain life-time. Beyond this timescale, the BIP will explore the AN at much larger length scales, resulting in a different, more diffusive-like dynamics.

Our observations do not indicate a strong link between the dynamics of BIP and the microtubules. Already, evidence against such a link came from our previous study [32], where it was concluded that EG are intimately connected to MT. Together with the enormous difference in MSD amplitude for EG and BIP, this excludes the possibility that also the BIP are linked to microtubule networks. The difference in microenvironment for BIP and EG becomes even more apparent when comparing the effects of drugs. Taxol, which had a strong effect on the MSD of EG, did not produce a significant change in the dynamics of BIP, while Latrunculin that dramatically altered the MSD of BIP, did not significantly affect the MSD of EG. The only observation which does not directly fit with our hypothesis that BIP are embedded in the actin network is the Nocodazole intervention, which needs to be addressed in the future.

\subsubsection{Driving forces for BIP}

Based on our observations we are unable to draw hard conclusions about the mechanism(s) that drive the motions of BIP. The much lower motion amplitudes compared to EG, seem to rule out that BIP are likewise driven by bending fluctuation of the MT. The insensitivity of the MSD of BIP to the Taxol intervention also suggests that indirect excitation, in which the AN transmits the MT fluctuations to the BIP, is unlikely.

Our results could still be consistent with acto-myosin contractility (AMC) as a driving force. The superdiffusive behavior observed after the LA intervention could in principle be due to AM driven contractions of the remaining actin network. And to the extent that the effect of Blebbistatin can be ascribed to the 
annihilation of AMC, the decrease in MSD could be explained by a loss of AMC. Finally, the effect of Nocodazole could also be seen in the light of the reported increase in actomyosin contraction force [16,17].

However as already stated in Sec. 3.2., the complexity of the cellular response can also obscure the relation between the primary effect of a drug and the change in MSD. Hence, additional evidence will be needed to resolve the question concerning driving forces.

\subsection{Conclusions and outlook}

Successful injection of many particles per cell allowed us to perform a systematic study of the dynamics of BIP in Hmec-1 cells. The signature of the MSD of BIP was entirely different than that earlier found for EG. Drug interventions indicated that (unlike EG, which occur close to microtubules), BIP are predominantly embedded in the actin network. This network behaves qualitatively as a viscoelastic liquid, with cage trapping at short lag times and more diffusive-like behavior at longer times. The mechanism by which the BIP are driven could not be conclusively revealed from the present work.

The fact that intracellular particle MSD are very sensitive to drug-induced changes in the state of the cell, confirms the potential for eventually using intracellular PTR in clinical studies. Moreover, since BIP and EG are associated with specific cytoskeletal biopolymer networks, they could form a dual probe system for both applied and fundamental research into cellular mechanical behavior.

\section{Acknowledgements}

We are grateful to Frieder Mugele for discussions, and to Denis Wirtz and Liesbeth Pierson for advice on ballistic particle injection. We thank Andries van der Meer and André Poot for providing cell lines and cell culture training. This research was supported by the Cell Stress program of the $\mathrm{MESA}^{+}$Institute of Nanotechnology. 


\section{References}

[1] B. Alberts, A. Johnson, J. Lewis, M. Raff et al., Molecular biology of the cell, Garland Science, New York, 2002.

[2] C.P. Brangwynne, F.C. MacKintosh and D.A. Weitz, Force fluctuations and polymerization dynamics of intracellular microtubules, PNAS USA 104 (2007), 16128-16133.

[3] M.R. Bubb, A.M.J. Senderowicz, K.L.K. Duncan and E.D. Korn, Jasplakinolide, a cytotoxic natural product, induces actin polymerization and competitively inhibits the binding of phalloidin to F-actin, J. Biol. Chem. 269 (1994), 14869-14871.

[4] M.R. Bubb, I. Spector, B.B. Beyer and K.M. Fosen, Effects of Jasplakinolide on the kinetics of actin polymerization: an explanation for certain in vivo observations, J. Biol. Chem. 275 (2000), 5163-5170.

[5] J.C. Crocker and D.G. Grier, Methods of digital video microscopy for colloidal studies, J. Colloid Interface Sci. 179 (1996), 298-310.

[6] J.C. Crocker and B.D. Hoffman, Multiple-particle tracking and two-point microrheology in cells, Methods Cell Biol. 83 (2007), 141-178.

[7] S.E. Cross, Y.S. Jin, J. Rao and J.K. Gimzewski, Nanomechanical analysis of cells from cancer patients, Nat. Nanotechnol. 2 (2007), 780-783.

[8] M.H.G. Duits, Y.Li, S.A. Vanapalli and F. Mugele, Mapping of spatiotemporal heterogeneous particle dynamics in living cells, Phys. Rev. E. 79 (2009), 051910.

[9] J. El-Ali, P.K. Sorger and K.F. Jensen, Cells on chips, Nature 442 (2006), 403-411.

[10] F. Gittes, B. Schnurr, P.D. Olmsted, F.C. MacKintosh and C.F. Schmidt, Microscopic viscoelasticity: Shear moduli of soft materials determined from thermal fluctuations, Phys. Rev. Lett. 79 (1997), 3286-3289.

[11] J. Guck, S. Schinkinger, B. Lincoln, F. Wottawah et al., Optical deformability as an inherent cell marker for testing malignant transformation and metastatic competence, Biophys. J. 88 (2005), 36893698 .

[12] W. Gutkowski and T.A. Kowalewski, Mechanics of the 21st century, Springer, Warsaw, Poland, 2005.

[13] B.D. Hoffman, G. Massiera, K.M.V. Citters and J.C. Crocker, The consensus mechanics of cultured mammalian cells, PNAS USA 103 (2006), 10259-10264.

[14] http://www.physics.emory.edu/ weeks.idl/.

[15] D.E. Ingber, Mechanobiology and diseases of mechanotransduction, Ann. Med. 35 (2003), 564-577. 
[16] M.S. Kolodney and E.L. Elson, Correlation of myosin light chain phosphorylation with isometric contraction of fibroblasts, J. Biol. Chem. 268 (1993), 23850-23855.

[17] M.S. Kolodney and E.L. Elson, Contraction due to microtubule disruption is associated with increased phosphorylation of myosin regulatory light chain, PNAS USA 92 (1995), 10252-10256.

[18] M. Kovacs, J. Toth, C. Hetenyi, A.M. Csizmadia and J.R. Sellers, Mechanism of Blebbistatin inhibition of myosin II, J. Biol. Chem. 279 (2004), 35557-35563.

[19] A. Lau, B.D. Hoffman, A. Davies, J.C. Crocker and T.C. Lubensky, Microrheology, stress fluctuations, and active behavior of living cells, Phys. Rev. Lett. 91 (2003), 198101.

[20] G.Y.H. Lee and C.T. Lim, Biomechanics approaches to studying human diseases, Trends Biotechnol. 25 (2007), 111-118.

[21] J.S. Lee, P. Panorchan, C.M. Hale, S.B. Khatau et al., Ballistic intracellular nanorheology reveals ROCK-hard cytoplasmic stiffening response to fluid flow, J. Cell Sci. 119 (2006), 1760-1768.

[22] T.G. Mason, K. Ganesan, J.H.V. Zanten, D. Wirtz and S.C. Kuo, Particle tracking microrheology of complex fluids, Phys. Rev. Lett. 79 (1997), 3282-3285.

[23] T.G. Mason, Estimating the viscoelastic moduli of complex fluids using the generalized Stokes-Einstein equation, Rheol. Acta 39 (2000), 371-378.

[24] J.L. McGrath, J.H. Hartwig and S.C. Kuo, The mechanics of F-actin microenvironments depend on the chemistry of probing surfaces, Biophys. J. 79 (2000), 3258-3266.

[25] D. Mizuno, C. Tardin, C.F. Schmidt and F.C. MacKintosh, Nonequilibrium mechanics of active cytoskeletal networks, Science 315 (2007), 370-373.

[26] A.L. Paguirigan and D.J. Beebe, Microfluidics meet cell biology: bridging the gap by validation and application of microscale techniques for cell biological assays, Bioessays 30 (2008), 811-821.

[27] P. Panorchan, J.S. Lee, B.R. Daniels, T.P. Kole et al., Probing cellular mechanical responses to stimuli using ballistic intracellular nanorheology, Methods Cell. Biol. 83 (2006), 115-140.

[28] P. Panorchan, J.S. Lee, T.P. Kole, Y. Tseng and D. Wirtz, Microrheology and ROCK. Signaling of human endothelial cells embedded in a 3D matrix, Biophys. J. 91 (2006), 3499-3507.

[29] P.B. Schiff and S.B. Horwitz, Taxol stabilizes microtubules in mouse fibroblast cells, PNAS USA 77 (1980), 1561-1565. 
[30] Y. Tseng, T.P. Kole and D. Wirtz, Micromechanical mapping of live cells by multiple-particle-tracking microrheology, Biophys. J. 83 (2002), 31623176.

[31] M.T. Valentine, Z.E. Perlman, M.L. Gardel, J.H. Shin et al., Colloid surface chemistry critically affects multiple particle tracking measurements of biomaterials, Biophys. J. 86 (2004), 4004-4014.

[32] S.A. Vanapalli, Y. Li, F. Mugele and M.H.G. Duits, On the origins of the universal dynamics of endogenous granules in mammalian cells, Mol. Cell. Biomech. 150 (2009), 1-16.

[33] S.A. Vanapalli, M.H.G. Duits and F.Mugele, Microfluidics as a functional tool for cell mechanics, Biomicrofluidics 3 (2009), 012006.

[34] K.M. Van Citters, B.D. Hoffman, G. Massiera and J.C. Crocker, The role of F-actin and myosin in epithelial cell rheology, Biophys. J. 91 (2006), 3946-3956.

[35] D. Weihs, T.G. Mason and M.A. Teitell, Bio-microrheology: A frontier in microrheology, Biophys. J. 91 (2006), 4296-4305.

[36] D. Weihs, T.G. Mason and M.A. Teitell, Effects of cytoskeletal disruption on transport, structure and rheology within mammalian cells, Physics of fluids, 19 (2007), 1031021-1031026.

[37] A.C. Yvon, P. Wadsworth and M.A. Jordan, Taxol suppresses dynamics of individual microtubules in living human tumor cells, Mol. Biol. Cell 10 (1999), 947-959. 


\title{
Chapter 6
}

\section{Intracellular Particle Tracking as a Tool for Tumor Cell Characterization}

\begin{abstract}
We studied the dynamics of two types of intracellular probe particles: ballistically injected latex spheres (BIPs) and endogenous granules (EGs), in tumor cell lines of differerent metastatic potential: breast tumor cells (MCF-7 malignant, MCF-10A benign) and pancreas adenocarcinoma (PaTu8988T malignant, PaTu8988S benign). For both tissue types and for both probes, the mean squared displacement (MSD) function measured in the malignant cells was substantially larger than in the benign cells. Only a few cells were needed to characterize the tissue as malignant or benign based on their MSD, since variations in MSD within the same cell line were relatively small. These findings suggest that intracellular particle tracking (IPT) can serve as a simple and reliable method for characterization of cell states obtained from a small amount of cell sample. Mechanical analysis of the same cell lines with Atomic Force Microscopy (AFM) in force-distance mode, revealed that AFM could distinguish between the benign and malignant breast cancer cells but not for the pancreatic tumor cell lines. This underlines the potential value of IPT as a complementary nanomechanical tool for studying cell state dependent mechanical properties.
\end{abstract}

This chapter has been accepted for publication in Journal of Biomedical Optics (2009). 


\subsection{Introduction}

Tumor cells can be characterized by different states reflecting the potential for rapid growth and metastasis. These properties are essential for a disease prognosis and a selection of treatment options. Usually tumor cells from surgery specimens or biopsies are characterized by microscopy after cell staining. Recent developments also use molecular or physical cell properties. One group of such methods is the in vitro microscopic analysis of small amounts of living cells, isolated from tumor samples.

Different criteria can then be used to distinguish between for example malignant and benign cancer cells. A recent addition to the spectrum of microscopic in vitro techniques is the characterization of cancer cells via their mechanical properties (1-3). This new direction in cancer research connects well to the emerging fields of mechanobiology and nanomechanical medicine. Especially the latter field capitalizes on the strong relation between the health state of the cell, and properties like its elastic stiffness or its viscoelastic spectrum. Recent studies have indicated that besides cancer also a variety of other diseases are linked to the changes of cell mechanical properties (4).

An excellent example of a nanomechanical technique is Atomic Force Microscopy (AFM). This technique was successfully used by Cross et al $(5,6)$ on populations of individual cells, to distinguish between healthy and malignant tissue from the lung, breast and pancreas. Significant differences in elastic modulus were found also for (populations of) cells that were not distinguishable by morphology. Also Li et al (7) used AFM to distinguish between benign and malignant breast cancer cells. They reported a viscoelastic response with different magnitude for the two types. However in spite of these proven capabilities, AFM remains a time consuming and complex technique. The main experimental challenges are the alignment of the tip with respect to the cell (8), the softness of the cell (9) and the lack of control over local strain (10).

In this paper we will explore an alternative microscopic technique, which we will call intracellular particle tracking (IPT) for the sake of convenience. IPT is not generally known under this name, but captures the study of either endogenous or ballistically injected particles (BIPs) inside living cells (11-18). The use of BIPs was introduced by the group of Wirtz and is known as Ballistic Intracellular Nanorheology (BIN) $(11,15,16)$. In IPT, video microscopy is used to track the motions of particles residing in the cytoplasm. Quantification of these motions via the time dependent mean squared displacement (MSD) then allows to study 
the type of dynamic behavior (from sub- to superdiffusive) as well as the amplitude of the motions. The precise interpretation of these motions may differ from case to case. Several papers $(15,16,19)$ reported on BIN as a tool to measure intracellular viscoelasticity, while other studies $(20,21)$ showed evidence that also molecular motors may contribute to particle motion. In anticipation of the latter, also a method of eliminating the ATP driven processes has been proposed (13). These various findings indicate that IPT should not be regarded as a nano-mechanical tool by default. However, one would still expect that a change in the cytoskeletal mechanics (like the softening of a cancer cell) also becomes manifest as a change in the MSD. Then having MSD curves for reference states (e.g. healthy cell, benign or malignant tumor cell) might allow to characterize the cellular state from the MSD.

To explore this potential, we studied cancer cells of different origin (breast and pancreas cancer) with IPT. Comparisons between the MSDs of benign and malignant cancer cells were made for both cell types, using two kinds of probe particles: 1. endogenous granules (EGs), which can be visualized with phase contrast microscopy, and 2. ballistically injected latex particles (BIPs) with carboxylate groups at their surface and a fluorescent core to facilitate visualization. Both probes are attractive candidates for IPT applications and have been used in previous IPT studies $(12,14,17)$. To assess the utility of IPT in comparison to other methods for mechanical diagnosis, we also performed elasticity measurements on the same cells using AFM in force-distance mode.

We here show that for both breast and pancreas cancer cells, and for both EGs and BIPs as probes, strong differences in MSD are found for benign and malignant tumor cells. Also we can demonstrate that AFM and IPT inherently measure different biomechanical aspects of the same cell, and could hence serve as complementary techniques.

\subsection{Material and methods}

\subsubsection{Cell Culture}

Human pancreas adenocarcinoma PaTu8988S (PA-S) and PaTu8988T (PA-T) cells were established from the same pancreatic tumor (22) and obtained from DSMZ (Germany). Cells were cultured in Dulbecco's Modified Eagle Medium (DMEM) containing 5\% Fetal Bovine Serum, 5\% horse serum and 1\% (2mM) Lglutamine (DSMZ, Germany). Human breast epithelial adenocarcinoma MCF-7 cells were cultured in RPMI1640 (Lonza) containing 10\% FCS, $1 \%(2 \mathrm{mM}) \mathrm{L}-$ glutamine, $10 \mu \mathrm{g} / \mathrm{ml}$ insulin, $1 \mathrm{mM}$ sodium pyruvate and non-essential amino 
acids. Human breast fibrocystic epithelial cells MCF-10A were cultured in Endothelial Growth Medium 2 (EGM-2) (Lonza). Cells to be analyzed with AFM and intracellular particle tracking (IPT) were grown on a Delta-T culture disk (Bioptechs, USA) until they reached 80 - 100\% confluency, and were kept under physiological conditions $\left(37^{\circ} \mathrm{C}, 5 \% \mathrm{CO} 2\right)$ until the measurements were completed.

\subsubsection{Nanomechanical measurements with AFM}

Force-Distance (F-D) curves were obtained using a home-built AFM head. This AFM, described in ref (23), uses the reflection of a laser beam for detection and contains a goggle for liquid operation. To extend the $(\mathrm{X}, \mathrm{Y}, \mathrm{Z})$ translation ranges to $100 \mu \mathrm{m}$ in each direction, it was mounted on a piezostage (Physik Instrumente) that in turn was mounted on the table of an inverted microscope (Nikon eclipse TE300). In our application, displacements of the cantilever base were controlled by a Nanoscope III controller (Veeco). Cells were pre-stained with DiI (Invitrogen, Breda, the Netherlands) to enable clear distinction of nuclei and membrane, as needed for the vertical and lateral positioning of the tip with respect to the cell. The latter was achieved using a combination of transmission, reflection and epifluorescent imaging mode (see Fig. 6.1.1 for an impression).

All experiments were done using silicon nitride cantilevers (Microlevers) having an experimentally determined spring constant $(24)$ of $0.03 \mathrm{~N} / \mathrm{m}$ and a pyramidal tip with a half opening angle of $15^{\circ}$. For each cell line, 3-4 different cells were studied, measuring 50-100 F-D curves per cell. Measurements were always done on top of the nuclear area. The typical force was $4-10 \mathrm{nN}$, corresponding to a typical indentation of $0.5-1.0 \mu \mathrm{m}$, which is small compared to the height of the cells. The F-D curves were also measured as a function of loading rate, covering $0.05,0.1,0.5,1.0$ and $2.0 \mathrm{~Hz}$, with each half-cycle corresponding to a travel of $2.5 \mu \mathrm{m}$ by the cantilever base. Apparent Young's moduli $E^{*}$ were obtained by fitting a modified Hertz model (25) to the data. Here the Poisson ratio of the cell was assumed to be 0.5 and the finite thickness of the cytoplasm between the membrane and the nucleus was not taken into account.

\subsubsection{Intracellular Probes}

We used two probes - ballistically injected particles and endogenous granules. The EGs were confirmed to be predominantly lipid droplets as proven by staining with Nile Red (Invitrogen). These granules have a mean diameter $\approx 0.5$ $\mu \mathrm{m}$, and appear as dark objects under phase contrast microscopy. The typical number of EGs per movie recorded was 20-50, which was sufficiently high for 
getting MSD's with a good signal/noise ratio. The particles were generally distributed evenly over the perinuclear cytoplasm.

BIPs can be chosen in different sizes and surface chemistries. Our BIPs were red-fluorescent carboxylated polystyrene particles with a diameter of $0.2 \mu \mathrm{m}$ (Invitrogen). These particles appear as bright spots in confocal fluorescent imaging, and as black spots in brightfield mode (see Fig. 6.1.4). Ballistic injection is needed to obtain enough particles per cell. In this case, homogeneous spreading of the probes is ensured by the injection method. We followed the procedure developed by Panorchan et al (15) and adopted for our case as described in (14). Briefly, a particle suspension at $2 \%$ weight concentration (Invitrogen) was diluted in pure ethanol and centrifuged at $8000 \mathrm{rpm}$ for $15 \mathrm{~min}$ in a Micromax RF microcentrifuge (IEC), after which the sediment was resuspended to reach again $2 \%$ weight concentration in ethanol. For efficient injection the particles were first spin-coated onto a macrocarrier disk of the Biolistic gun (He/PS 1000, BioRad). Ballistic bombardments were performed using optimized injection parameters (vacuum level, Helium pressure, carrier disk - cell sample distance). To prevent endocytosis of non-injected particles, samples were flushed with growth medium extensively after bombardment, replated and incubated for at least 3 hours before the particle tracking experiments. This procedure yielded typically 5-20 particles per cell.

\subsubsection{Particle tracking using optical microscopy}

Probe particles were visualized using the UltraView LCI10 system (Perkin Elmer), in which a Yokogawa spinning-disk confocal unit is combined with a Nikon Eclipse TE-300 microscope. A 100X (NA 1.3) oil immersion objective was used. BIPs were visualized in confocal fluorescence mode, while endogenous granules were imaged in phase contrast mode. In a typical movie, 2500 images were recorded with a 12-bit CCD camera (Hamamatsu) at $\sim 17 \mathrm{fps}$ for a typical duration of $150 \mathrm{~s}$. The image size was $87 \times 66 \mu \mathrm{m}$, which typically included 10-20 individual cells. The spatial resolution corresponding to the images was $0.13 \mu \mathrm{m} /$ pixel and the focal plane in which particles could be detected had a width of $\sim 1 \mu \mathrm{m}$. For each cell line, 10-20 measurements were done as visible in the field of view of the microscope. Particle tracking was performed using the available particle-tracking code written in IDL (26) and described in ref (27).

Excellent descriptions of the theoretical background and the methodology of particle tracking can be found in refs $(18,28,29)$. Briefly, the particles were localized per individual frame (typical accuracy: 10 15 nm) after which the in- 
plane (i.e. X, Y) displacements of the same particle were combined into a trajectory. From these trajectories the Mean Squared Displacement (MSD) vs. lagtime function is calculated by averaging over particles and time steps:

$$
\Delta r^{2}(\tau)=\left[<\left(x_{p}(t+\tau)-x_{p}(t)\right)^{2}+\left(y_{p}(t+\tau)-y_{p}(t)\right)^{2}>\right]
$$

where $x_{p}(t)$ and $y_{p}(t)$ correspond to the position of particle $\mathrm{p}$ at time $\mathrm{t}$, the brackets $<$ indicate an averaging over all times $t$ and [] represents averaging over all particles $\mathrm{p}$. More details on averaging issues and Signal/Noise ratio can be found in (12). In our case the probe particles were compartmentalized in cells, which in principle allow to calculate average MSDs per cell. In this study, averaging was done over all 10-20 measurements that were recorded per cell line unless mentioned otherwise.

\subsection{Results and Discussion}

\subsubsection{Intracellular Particle Tracking}

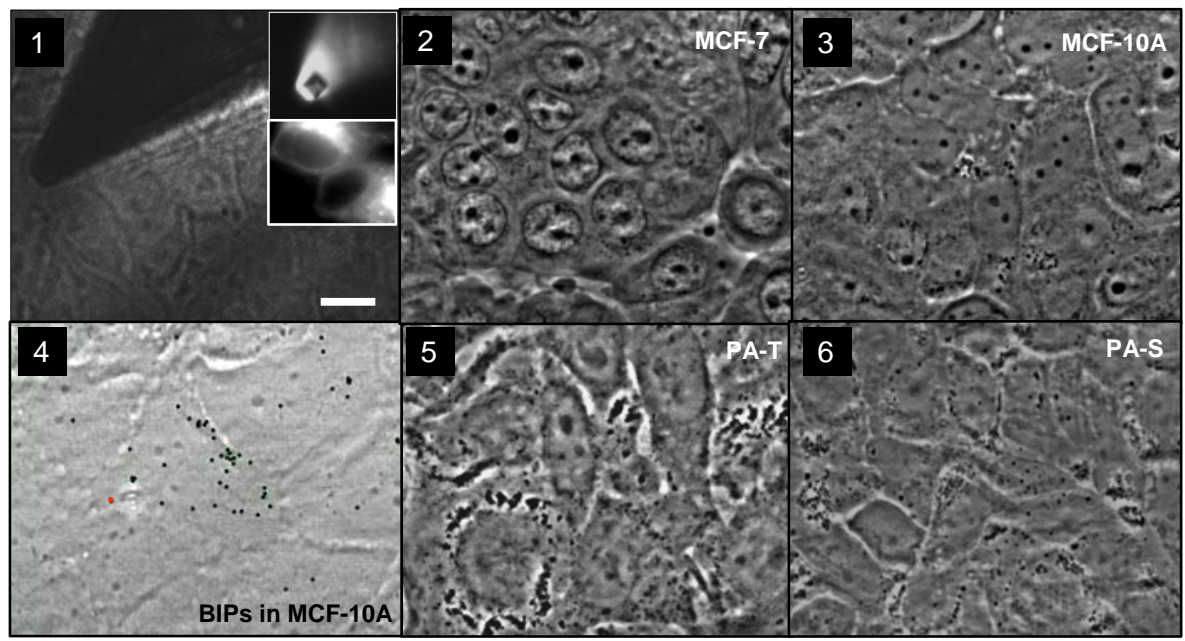

Fig. 6.1, phase contrast images of pancreatic and breast tumor cells. 1, Image demonstrating the AFM tip aligned over the central (nuclear) region of a cell. The insets show the pyramidal tip in reflective imaging mode and MCF-7cells in epifluorescent imaging mode. 2,3,5,6, Typical morphology of a confluent monolayer of MCF-7, MCF-10A, PA-T and PA-S cells. 4, Illustration of the intracellular locations of BIPs (here visualized as black filled circles) in a monolayer of MCF-10A cells. The scale bar applies to all images and measures $10 \mu \mathrm{m}$. 
In Fig. 6.1, phase contrast microscopy images are shown for each of the studied cell lines. In all cases the degree of confluency was between 80 and $100 \%$. For cell densities significantly below $100 \%$, benign cells could be recognized by their tendency to form tightly bound colonies, while the malignant pancreas tumor cells could be recognized by their relatively high growth rate. However once the cells had reached the (near) confluent state, they could no longer be distinguished by cell morphology. Fig. 6.1 shows the morphologies of the benign and malignant cells in the (near) confluent state.

The results of the IPT experiments with the four cell lines are summarized in Fig. 6.2. Each subfigure shows the total-average MSD obtained by sub-averaging first over all particles in the same image-time series (capturing 10-20 cells simultaneously), and subsequently over 10-20 such movies taken at different locations in the culture dish. In Fig. 6.2.1 also the sub-average results are shown (for BIPs in MCF cells). Importantly, in some cell lines, not only the totalaverage MSDs of benign and malignant cells are strongly different, but also the sub-average MSDs clearly reveal to which family of curves (i.e. benign or malignant) they belong, for example the dynamics of BIPs in breast tumor cells and dynamics of EGs in pancreas tumor cells. The same observation was made for the other cell/probe combinations (not shown). Taken together, these findings suggest that only a few cells could be sufficient to determine whether the sampled tissue contains benign or malignant cells. Moreover, since the amplitude difference between the MSDs of malignant and benign cells is very large (up to an order of magnitude) and also persists over a large range of lagtimes (two decades), the outcome of such a test would not critically depend on the precise time scale (range) of the experiment. 

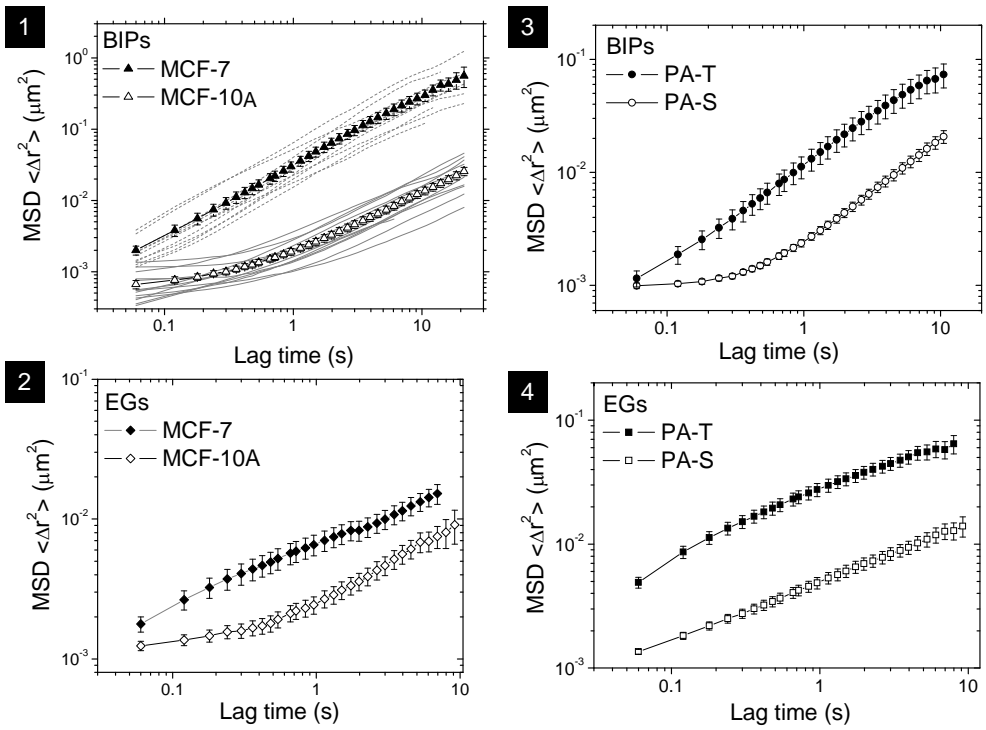

Fig. 6.2, Mean square displacement versus lagtime functions of EGs and BIPs in two pairs of tumor cells under physiological conditions. Filled symbols: malignant tumor cells, open symbols: benign tumor cells. Results are averaged over 10-20 measurements in each cell line. Error bars reflect standard errors. 1, Dashed/Solid lines: single-movie measurements of MCF-7/MCF-10A.

The results of Fig. 6.2 raise two questions, namely: 1) what could be the physical origin of the different MSDs found for the same type of probe particles in the benign and malignant cells, and 2) why the MSDs for EGs and BIPs inside the same cell are so different.

Regarding the first question, it is clear from the literature on IPT and BIN $(15,16$, 19) that intracellular particles of colloidal size $(100 \mathrm{~nm}-1 \mu \mathrm{m})$ probe the constraints presented by the visco-elastic polymer network that embeds them or that binds to them. Then the erratic motions of the particles can be seen as the result of a driving force that excites the particle motion, and a viscoelastic response force that provides damping. In case the driving forces would be purely thermal collisions, the MSD would represent local viscoelastic properties. However in a living cell it cannot be ruled out that also molecular motor driven processes are responsible for particle motion. This means that (in the absence of additional information) one can not in principle say to what extent the larger MSDs for the malignant cells (found for both probes and both cell types) are due to a lower viscoelastic resistance or due to stronger driving forces. As a note we add here that in a recent study (21) it was found that for lagtimes $<0.1 \mathrm{~s}$, 
nonthermal contributions were negligible. In that light, our MSD measurements at short lagtimes would suggest that (except for BIPs in pancreatic tumor cells) for malignant cells the viscoelastic resistance to deformation is smaller. This is also what would be expected from the physiological argument that malignant cells are more deformable because of their metastatic nature.

Concerning the second question, the answer has to be that the two probes occur in rather different local micro-environments (i.e. polymer network surroundings). This is not surprising, considering that the surface chemistry (i.e. the groups that are exposed to the cytoplasm) of EGs and BIPs is expectedly also very different: for EGs this is the least known but most probably a variety of (surface active) proteins (30), while for BIPs it is known to be carboxylate groups only. Moreover, in previous studies $(14,17)$ we also found that while the dynamics of EGs is closely linked to that of the microtubules, the motions of BIPs are strongly correlated to the actin network. Due to these different microenvironments, the driving forces and/or viscoelastic resistance are very different. The foregoing analysis rises as an additional question, how measurements of intracellular MSDs could be connected to the viscoelastic properties of the whole cell. It is generally believed that the mechanics of the cell is dominated by the cytoskeleton. Taken together with the notions that BIPs are embedded in the actin network, and that BIPs are not very obviously driven by ATP (unlike EGs) $(14,16)$, it is then suggested that the MSDs of the BIPs should give the closest representation of the cell's viscoelastic properties. From the shapes of the MSD curves for BIPs it seems that the benign cells behave like a visco-elastic liquid (similar to a Maxwell fluid, see (31) for examples) whereas the malignant cells appear more fluid like.

\subsubsection{Atomic Force Microscopy}

Considering the expected relative softness for the malignant cells, and the findings with IPT, it is interesting to examine measurements made on the same cells with AFM in force-distance mode. Anticipating a visco-elastic response, we measured the curves as a function of loading rate. A typical series of forceindentation curves is given in the inset of Fig. 6.3. All curves are fairly well described by a parabolic equation, even though this is only expected for quasistatic indentation of an elastic material with a pyramidal tip (8). Fitting the modified Hertz model (25) allowed to obtain an apparent Young's modulus E* from each of the force-indentation curves. The results are summarized in Fig. 6.3.

First of all, we note that the difference between the values of $E^{*}$ for the malignant and benign cells (at the same loading rate) is large for the breast 
cancer cells, but insignificant for the pancreatic cancer cells. This is all the more remarkable, considering that the $\mathrm{E}^{*}$ values for the malignant breast and pancreatic cells are very similar. This circumstance should make the measurements very comparable, and make it unlikely that differences in the AFM experiment (rate and range of indentation, local strain fields) play a role.

We thus believe that the $\mathrm{E}^{*}$ data do capture the presence or absence of differences in cell stiffness. To what extent the $\mathrm{E}^{*}$ measurements are truly quantitative will be discussed below. We conclude that at least under the conditions that we used, AFM is able to distinguish the MCF-7 from the MCF10A cells, but not the PA-S cells from the PA-T cells.

It is also observed in Fig. 6.3 that for all cell types, the apparent elastic modulus shows an increase with loading rate. Considering that the loading rate varies over almost two decades, the factor 2 increase in $\mathrm{E}^{*}$ seems modest. To examine the possibility that the loading rate dependence is due to hydrodynamic forces, we also performed measurements on hard substrates in water. Even close to the hard surface, no forces with a magnitude comparable to the experiments with cells could be found. This suggests that the trends in Fig. 6.3 are indeed due to the visco-elastic nature of the cells.
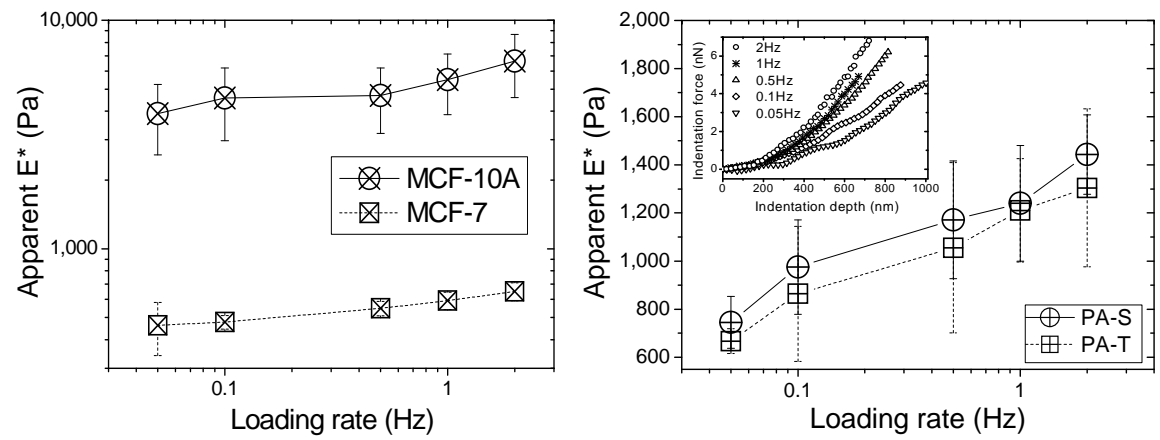

Fig. 6.3, Loading rate dependent apparent elastic Young's modulus of two pairs of tumor cells under physiological conditions. Left: breast cancer cells, MCF$10 A$ vs. MCF-7. Right: pancreatic tumor cells, PA-S vs. PA-T. The inset shows the indentation force versus indentation depth curve of a single PA-T cell at different loading rates.

We now compare our results with literature findings. It is interesting to note that qualitatively similar results were obtained for the MCF-7 and MCF-10A cells by $\mathrm{Li}$ et al (7). While their loading rate dependence of $\mathrm{E}^{*}$ was very similar, the magnitudes of $E^{*}$ found by them are a factor 5 lower than ours. It is not evident 
where this difference originates from. Most probably they are due to differences in measurement. $\mathrm{Li}$ et al used a colloidal tip and limited the indentation range to $500 \mathrm{~nm}$ or less. This implies a larger contact surface and lower strains in their experiments. Also the finite thickness effect (32) could have affected their measurements less than ours. Each of these differences could cause significant effects on the values obtained for $\mathrm{E}^{*}$.

Moreover besides these aspects which would already apply for homogeneous viscoelastic bodies, there is also the fact that mammalian cells are composite bodies, which in the simplest case should consist of a mechanically distinct cortex and cytoplasm (31). To which extent these two elements are probed, depends on the strain field, which is not easy to assess in a composite body. Hence the difference between the two measurements (i.e. Li (7) vs. ours) may also be due to different relative contributions of the cortex and cytoplasm.

On the basis of these considerations it cannot be entirely ruled out, that under different conditions it might still be possible to distinguish between PA-S and PA-T with AFM. On the other hand it can also be concluded that also with AFM it is challenging to obtain a quantitative mechanical characterization of the tumor cells.

\subsection{Further considerations}

\subsubsection{Comparison of IPT and AFM}

Although both intracellular particle dynamics as measured with IPT and the apparent Young's modulus as measured with AFM are related to mechanical properties of the cell, it has also become clear that a direct comparison of the IPT and AFM results at the level of viscoelastic properties is not feasible. This is further illustrated by a comparison of the apparent elastic modulus of the MCF$10 \mathrm{~A}$ cells as obtained from an (over) simplistic interpretation. Using AFM in the limit of small loading rates, an $\mathrm{E}^{*}$ of $\approx 4 \mathrm{kPa}$ is obtained (see Fig. 6.3). In contrast, application of the generalized Stokes-Einstein relation (that assumes thermal driving forces) (29) to the MSD plateau found with BIPs (Fig. 6.2), yields an apparent $\mathrm{E}^{*}(=4 * \mathrm{G}$ for incompressible materials $)$ of $40 \mathrm{~Pa}$.

This enormous difference suggests that even if the conditions for the IPT and AFM experiments could be chosen such that well-defined mechanical properties would be measured, different viscoelastic behaviors would still be found with the two techniques. Considering the complex mechanical architecture of the cell, this is also not surprising. While IPT probes the dynamics of the intracellular 
cytoskeleton, AFM probably probes a combination of the elastic shell and the viscoelastic interior of the cell. This means that even under conditions optimized for measuring purely mechanical properties, different mechanical aspects of the same cell are measured. In this respect the two techniques could be seen as complementary to each other.

Another aspect is the degree of biochemical selectivity which can be achieved with the two techniques. Most AFM studies have used an inert pyramidal tip (9) or colloidal particle $(7,10)$ to probe the cell just like it would probe any other viscoelastic body. However, also measurements that are more specifically aimed at the cytoskeleton are possible, e.g. by using a functionalized tip that binds to integrin receptors on the cell surface (33). With IPT, possibilities for studying (processes related to) cell mechanics with or without molecular sensitivity seem even larger. This is illustrated by several previous BIN studies using (weakly interacting) carboxylate, (strongly binding) amino (16) and (inert) PEG (34) surface coatings, and by our earlier studies on EGs and BIPs in Hmec-1 cells which gave qualitatively different MSDs that were ascribed to different components of the cytoskeleton $(14,17)$.

On the technical level, AFM is clearly a more demanding technique than IPT, when applied to living cells. Alignment of the tip with respect to the cell requires a good optical access of both tip and cell, and an accurate positional control combined with a large range. Since cells are soft objects, they require soft cantilevers and hence the sensitivity to acoustic noise is high (9). And finally also the transmission of the laser beam (used for measuring cantilever deflection) can be compromised by liquid turbidity caused by either the cell medium or cell debris. In contrast, IPT just requires that the cells adhere to a transparent substrate, that the probe particles are visible with a microscope, and show enough motion to obtain an MSD. In case of BIPs, an extra bombardment step is needed, in return for a very well-defined particle size and surface chemistry.

\subsubsection{Implementation perspectives for IPT}

The present study is, as far as we know, the first application of IPT to characterize benign and malignant cancer cells. We applied it to cell lines originating from two different tissues, breast and pancreas, and found clear differences between the respective MSDs. In the case of isogenic pancreas cells IPT was even able to distinguish malignant from benign cell lines, whereas this was not possible with our AFM measurement. Whether IPT would be more broadly applicable for cell characterization would require additional experiments 
with tumor and healthy cells and tissues from the same donor, but the perspectives seem good, for different reasons:

1. Malignant cells are mechanically softer, and have a different structural organization of their cytoskeleton (7). If an intracellular probe particle is sensitive to either one or both aspects, its MSD will very likely be different as well. Even with endogenous probes (which have a less well-defined surface chemistry than BIPs), malignant cells could be distinguished from benign ones. This is ascribed to their occurrence near microtubules (MTs) (17).

2. The use of more than one intracellular probe may increase the reliability of tumor characterization. For example, if the probes are sensitive to different intracellular microenvironments, then their MSDs could provide complementary information (e.g. about changes in the actin network and MTs in case of our BIPs and EGs, respectively). The results with different probes can also corroborate each other in the sense of a cell characterization.
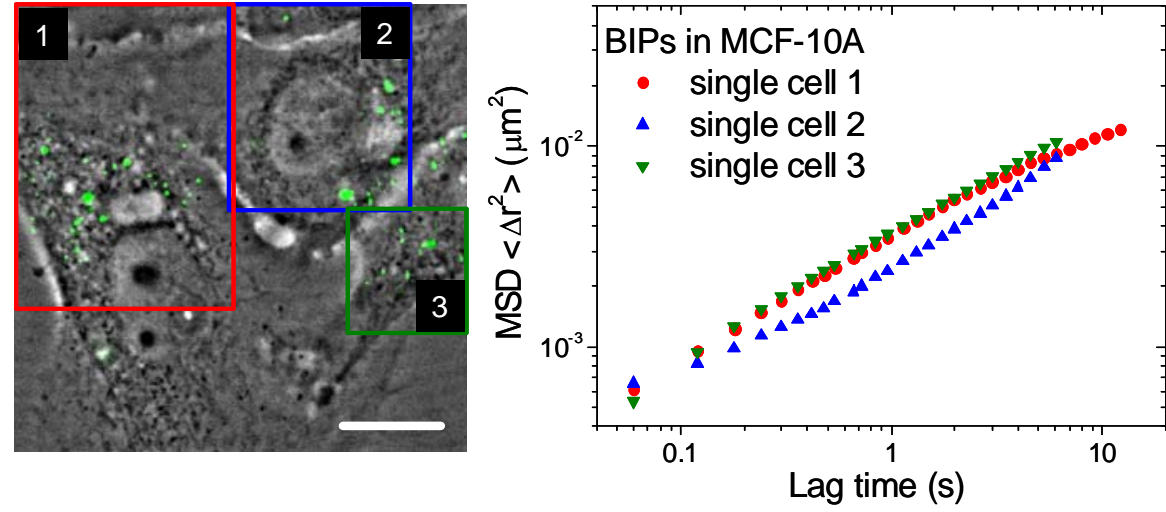

Fig. 6.4, Dynamics of BIPs in individual cells. Left: combination of phase contrast image of a monoconfluent layer of MCF-10A cells and confocal fluorescence image of the BIPs inside the cells. Right: Mean square displacement versus lag time function in cells 1, 2, 3. Scale bar is $10 \mu \mathrm{m}$.

3. Characterization could be based on a small number of cells. As already illustrated in Fig. 6.2.1, in some cases, only a few cells were sufficient to categorize cells as rather malignant or benign. To explore this further, we compared MSDs of BIPs measured in three individual MCF-10A cells (in the same confluent layer). As shown in Fig. 6.4, the differences between the MSDs are rather small (compared to the differences in Fig. 6.2.1). This illustrates that in 
favorable cases; even individual cells could be used to characterize a cell population.

4. IPT can be easily combined with drug intervention studies. Demonstrations hereof have already been given in fundamental studies $(13,14,17,35)$. The next step would be the use of IPT in the development of preclinical in vitro models for the drug candidate analysis.

\subsection{Summary}

The Mean Squared Displacement function measured with Intracellular Particle Tracking cannot be generally linked to a well-defined mechanical property of the cell, but was found to be very sensitive to differentiation state of cancer cells, for two tissue types and for two different kinds of probe particles. Cell state could be simply identified by comparison with reference MSD curves for the same probe. This indicates a good potential for using IPT to diagnose cells whose health state is reflected in their mechanical properties. As such IPT appears to be a valuable nanomechanical tool which is complementary to AFM.

\section{Acknowledgements}

This research was supported by the Cell Stress program of the MESA+ Institute of Nanotechnology and BMBF grants Cell@Nano and NanoCare. We are grateful to Denis Wirtz and Liesbeth Pierson for advice on ballistic particle injection. We thank Cock Harteveld and Mariska van der Weide for technical support, and Istvan Vermes, Andries van der Meer and Frieder Mugele for discussions.

\section{References}

1. S. Suresh, "Biomechanics and biophysics of cancer cells," Acta Biomaterialia 3,413-438 (2007)

2. S. Suresh, "Elastic clues in cancer detection," Nanomedicine 2, 748-749 (2007)

3. S. Suresh, J. Spatz, J. P. Mills, A. Micoulet, M. Dao, C. T. Lim, M. Beil and T. Seufferlein, "Connection between single-cell biomechanics and human disease states: gastrointestinal cancer and malaria," Acta Biomaterialia 1,15-30 (2005)

4. G. Y. H. Lee and C. T. Lim, "Biomechanical approaches to studying human diseases," Trends Biotechnol. 25(3), 111-118 (2007) 
5. S. E. Cross, Y. S. Jin, J. Y. Rao and J. K. Gimzewski, "Nanomechanical analysis of cells from cancer patients," Nature nanotech. 2, 780-783 (2007)

6. S. E. Cross, Y. S. Jin, J. Tondre, R. Wong, J. Y. Rao and J. K. Gimzewski, "AFM-based analysis of human metastatic cancer cells," Nanotechnology 19, 384003 (2008)

7. Q. S. Li, G. Y. H. Lee, C. N. Ong and C. T. Lim, "AFM indentation study of breast cancer cells," Biochem. Biophys. Res. Commu. 374, 609-613 (2008)

8. H. J. Butt, B. Cappella and M. Kappl, "Force measurements with the atomic force microscope: Technique, interpretation and applications," Surf. Sci. Rep. 59(1-6), 1-152 (2005)

9. M. Radmacher, "Studying the mechanics of cellular processes by atomic force microscopy," Meth. Cell. Biol. 83, 348-369 (2007)

10. G. T. Charras and M. A. Horton, "Determination of cellular strains by combined atomic force microscopy and finite element modeling," Biophys. J. 83(2), 858-879 (2002)

11. Y. Tseng, T. P. Kole and D. Wirtz, "Micromechanical mapping of live cells by multiple-particle-tracking microrheology," Biophys. J. 83(6), 3162-3176 (2002)

12. M. H. G. Duits, Y. Li, S. A. Vanapalli and F. Mugele, "Mapping of Spatiotemporal Heterogeneous Particle Dynamics in Living Cells," Phys. Rev. E 79(051910), (2009)

13. B. D. Hoffman, G. Massiera, K. M. V. Citters and J. C. Crocker, "The consensus mechanics of cultured mammalian cells," PNAS USA 103(27), 10259-10264 (2006)

14. Y. Li, S. A. Vanapalli and M. H. G. Duits, "Dynamics of ballistically injected latex particles in living human endothelial cells," Biorheology, 46(4), 309-321 (2009)

15. P. Panorchan, J. S. Lee, B. R. Daniels, T. P. Kole, Y. Tseng and D. Wirtz, "Probing cellular mechanical responses to stimuli using ballistic intracellular nanorheology," Meth. Cell. Biol. 83,115-140 (2006)

16. Y. Tseng, T. P. Kole and D. Wirtz, "Micromechanical mapping of live cells by multiple-particle-tracking microrheology," Biophys. J. 83(6), 3162-3176 (2002)

17. S. A. Vanapalli, Y. Li, F. Mugele and M. H. G. Duits, "On the origins of the universal dynamics of endogenous granules in mammalian cells," Mol. Cell. Biomech. 79(051910), (2009)

18. D. Weihs, T. G. Mason and M. A. Teitell, "Bio-microrheology: A frontier in microrheology," Biophys. J. 91(11), 4296-4305 (2006) 
19. S. R. Heidemann and D. Wirtz, "Towards a regional approach to cell mechanics," Trends Cell Biol. 14(4), 160-166 (2004)

20. A. W. C. Lau, B. D. Hoffman, A. Davies, J. C. Crocker and T. C. Lubensky, "Microrheology, stress fluctuations, and active behavior of living cells," Phys. Rev. Lett. 91(19), 1981011-1981014 (2003)

21. D. Mizuno, C. Tardin, C. F. Schmidt and F. C. MacKintosh, "Nonequilibrium mechanics of active cytoskeletal networks," Science 315(5810), 370-373 (2007)

22. H. P. Elsasser, U. Lehr, B. Agricola and H. F. Kern, "Establishment and characterisation of two cell lines with different grade of differentiation derived from one primary human pancreatic adenocarcinoma," Virchows Archiv 61(5), 295-306 (1992)

23. K.O. Van der Werf, C. A. J. Putman, B. G. Degrooth, F. B. Segerink, E.H.Schipper, H. F. V. Hulst and J.Greve, "Compact stand-alone atomicforce microscope," Rev. Sci. Instrum. 64(10), 2892-2897 (1993)

24. R. Levy and M. Maaloum, "Measuring the spring constant of atomic force microscope cantilevers: thermal fluctuations and other methods," Nanotechnology 13, 33-37 (2002)

25. I. N. Sneddon, "The relation between load and penetration in the axisymmetric boussinesq problem for a punch of arbitrary profile," Int. J. Eng. Sci. 3, 47-57 (1965)

26. http://www.physics.emory.edu/ weeks.idl/,

27. J. C. Crocker and D. G. Grier, "Methods of digital video microscopy for colloidal studies," J. Colloid Int. Sci. 179, 298-310 (1996)

28. T. G. Mason, "Estimating the viscoleastic moduli of complex fluids using the generalized Stokes-Einstein equation," Rheol. Acta 39(4), 371-378 (2000)

29. T. A. Waigh, "Microrheology of complex fluids," Rep. Prog. Phys. 68, 685-742 (2005)

30. R. Bartz, W-H. Li, B. Venables, J. K. Zehmer, M. R. Roth, R. Welti, R. G. W.Anderson, P. Liu and K. D. Chapman, "Lipidomics reveals that adiposomes store ether lipids and mediate phospholipid traffic," J. Lipid Res. 48, 837-847 (2007)

31. C. T. Lim, E. H. Zhou and S. T. Quek, "Mechanical models for living cells - A review.," J. Biomech. 39(2), 195-216 (2006)

32. R. E. Mahaffy, S. Park, E. Gerde, J. Kas and C. K. Shin, "Quantitative analysis of the viscoelastic properties of thin regions of fibroblasts using atomic force microscopy," Biophys. J. 86(3), 1777-1793 (2004) 
33. Y. Verdier and B. Penke, "Binding sites of amyloid beta-peptide in cell plasma membrane and implications for Alzheimer's disease," Curr. Protein Pept. Sci 5(1), 19-31 (2004)

34. B. R. Daniels, B. C. Masi and D. Wirtz, "Probing Single-Cell Micromechanics In Vivo: The Microrheology of C. elegans Developing Embryos," Biophys. J. 90(12), 4712-4719 (2006)

35. C. Rotsch and M. Radmacher, "Drug-induced changes of cytoskeletal structure and mechanics in fibroblasts: an atomic force microscopy study, "Biophys. J. 78, 520-535 (2000) 


\section{Chapter 7: Summary and Outlook}

\subsection{Summary}

The goal of this thesis work was to assess 1) the capability of intracellular particle tracking to generate new insights about the micro environments of different intracellular probes, 2) the forces driving different probe particles in living cells, 3) the heterogeneity of the cytoskeleton as indicated by spatial and temporal variations in probe dynamics, and 4) the potential of intracellular particle tracking for biomedical applications. This approach was on one hand expected to contribute to the quest for 'ideal tracers' : particles that due to their surface biochemistry would reveal specific information like the dynamics of cytoskeletal components such as actin and microtubule networks, or the rheological behavior of the cytoskeleton. Besides these fundamental aspects, there were also expectations from a practical perspective: the research might stimulate the development of Intracellular Particle Tracking (IPT) as a biomechanics based diagnostic tool for cells. IPT could then be used to distinguish between healthy and diseased cells, and/or to explore the effects of clinically relevant drugs via their influence on the cytoskeleton.

In Chapter $1 \& 2$, the field of cell mechanics and the aim of this project were introduced. A detailed overview was given about the experimental approaches and the emphasis was put on IPT and AFM. In addition, materials and methods were used in our study were discussed in details.

In Chapter 3, we addressed the spatiotemporal heterogeneity in the dynamics of endogenous lipid granules in living Hmec-1 cells by analyzing a large set of individual particle trajectories. One motivation for this study was that heterogeneity could compromise the interpretation of MSDs, if these were based on just a few particles per cell sample. Besides that, assessing heterogeneities in intracellular dynamics was also interesting in its own right. The amplitude of the MSD was found to show strong variations between different particles. However, no evidence was found for correlations between MSD and intracellular location. This implies that an MSD that is representative for a single cell can be obtained by just averaging over enough particles per cell. This outcome also validated the use of averaged MSDs in comparative studies, as discussed in chapters 4 to 6 . Also the length- and timescales, over which the MSD amplitude remains correlated, were studied. Spatial correlations were found absent at least down to interparticle distances of 2 microns, indicating that the heterogeneities are highly 
local. Up to a time scale of 150 s, no important changes in MSD amplitude for the same particle were found.

In Chapter 4 and 5, we studied two kinds of intracellular probes: ballistically injected carboxylated latex particles (BIPs) and endogenous granules (EGs), in living human endothelial cells. For EGs, the MSDs showed a diffusive-like signature, which appears to be universal for mammalian cells. At reduced temperature $\left(24^{\circ} \mathrm{C}\right)$ the amplitude and lagtime dependence of the MSD could be fairly well described by theory for semiflexible polymers that undergo thermal fluctuations. Assuming that active forces are negligible at $24^{\circ} \mathrm{C}$ and that the EGs follow the motion of cytoskeleton polymers, it was indicated that the dynamics of the EGs might be linked to that of the microtubules (MTs). At physiological temperature $\left(37^{\circ} \mathrm{C}\right)$ the MSDs showed a much larger amplitude, which was hypothesized to be due to active forces (in spite of the diffusive-like MSD). These assumptions for the micro-environment and active driving forces for EGs could be corroborated by comparing MSDs after various chemical interventions. Here it turned out that EG dynamics was much more sensitive to changes in the MT network than to alterations in the actin network. Finally it was proposed that under physiological conditions, the dynamics of EGs can be explained by bending fluctuations of the MTs, caused by MT (de-)polymerization forces. All of these findings support the notion that EGs can be used as non-invasive probes for the dynamics of the MT network.

Our BIPs showed an entirely different dynamics inside the same cells. First of all the MSD amplitudes were much lower than for EGs. At short lag times the MSD vs time curves showed a quasi-plateau, which is the signature of particles that are trapped in a viscoelastic environment. At long lag times, the MSD was diffusivelike. The same drug interventions earlier used in the study of EGs, had an entirely different effect on the MSDs of the BIPs, and revealed that BIPs must be predominantly embedded in the actin network. Additional drug interventions aiming at disabling non-thermal forces, could not provide conclusive evidence about which forces (e.g. thermal or ATP dependent like actomyosin contractility) drive the BIPs motions. Taken together, these findings validate the use of BIPs to monitor the dynamic changes in the actin network.

From a microrheological point of view it was concluded that under standard physiological conditions, and at time scales of $0.1-10 \mathrm{~s}$, neither EGs nor our BIPs can safely be assumed to obey the Generalized Stokes Einstein Relation. It is not to be excluded however, that conditions can be found where the GSER does apply. Such conditions might occur at short enough lagtimes or after 
suitable physical or chemical interventions which selectively eliminate ATP dependent processes.

In chapter 6, we explored the use of IPT as a tool to characterize cancer cells as being malignant or benign, based on their MSD. Both EGs and BIPs were explored as probes, and the analysis was applied to both pancreatic and breast tumor cells. It was consistently found that in malignant cells, MSDs amplitudes were higher than in the benign cells. This illustrates the utility of IPT to provide a semi-quantitative measure of the tumor metastatic potential. Analyzing the same cells with Atomic Force Microscopy in force-distance mode revealed that the apparent elastic moduli of the malignant breast tumor cells were significantly lower than for the benign ones. However for the pancreatic cells the moduli of benign and malignant cells could not be distinguished. This illustrates the value of IPT also as a complementary technique. IPT was hence corroborated to have potential for applications in diagnosis, prognosis or pharmacology.

\subsection{Outlook}

Intracellular particle tracking offers a certain potential for biomechanics-oriented studies of living cells. The simplicity of the experiment, small amount of sample and the ability to track the remodeling of different cytoskeletal components (actin network and microtubules) make it an attractive addition to the spectrum of cell characterization methods. However it is also clear that further aspects have to be considered in assessing the potential of IPT compared to other methods, and in developing the method. Below, these aspects will be discussed along two lines.

\subsubsection{IPT for fundamental cell-mechanical studies}

1, Particles as tracers for cytoskeletal components.

The apparently selective embedding of carboxylated BIPs in the actin network and EGs in the microtubule network as found in this thesis, is promising and could be followed up using particles with even more specific surface chemistries. This could increase the selectivity of the embedding. Also the occurrence of binding between probes and cytoskeleton components could be studied, by using smaller particles. For particles smaller than the mesh size, (un)binding should drastically change their dynamics. Inherently, the use of attached probes particles will always be an indirect way of studying the dynamics and remodeling of the cytoskeleton. Therefore it will also have to be assessed under which conditions a direct real-time visualization of the actin and MT networks will be preferred. 
2, Particle tracking for intracellular microrheology.

Further study will be needed into the possibilities for avoiding or eliminating the contributions of active forces to the particle dynamics. Attempts to inactivate all ATP dependent processes, or more specifically acto-myosin contractility resulted in cytoskeletal modification as was found in this thesis. On the other hand, it seems that the exploration of this idea has only just begun. Knowledge on the effects (and optimal concentration and incubation time) of existing drugs will continue to grow, and new drugs will be discovered. Alternatively it could also be attempted to quantify the contributions of active forces to the particle dynamics, and obtain the passive rheological properties from an analysis which takes these contributions into account.

Finally, the merits of multiple particle tracking using video microscopy, as the used IPT method is often termed, will have to be compared to other microrheological methods. Active microrheology, with using magnetic fields or optical tweezers, does not suffer from ATP dependent contributions. And two-point microrheology is insensitive to probe-matrix interactions.

3, Study of biological processes through modification of BIPs surface chemistry. The large heterogeneity observed in BIPs dynamics gives a hint of the complexity of the biological processes that are sensed by carboxylated BIPs. Through modification of their surface chemistry, it might be possible to precisely target BIPs to specific components inside the living cells, also other than the main cytoskeletal components. Thus BIPs could also serve as intracellular biomarkers to study the biological processes.

\section{4, Bridging the gap of in vivo and in vitro particle tracking studies.}

Bottom-up approaches (in vitro synthetic polymeric network) and top-down approaches (in vivo measurement in living cells) are fundamentally different, yet also complementary to each other. Bottom-up approaches offer a simplified environment and well-controlled conditions, but crucially depend on how well they still capture the behavior inside real cells. Top-down approaches directly address the living cells but can bring serious interpretation issues. A large obstacle encountered in our top-down IPT experiments was, how to translate the MSDs measured with IPT, into material functions like viscoelasticity.

Particle tracking can be applied both in vivo and in vitro. As the in vitro systems are increasingly approaching the complexity of real life, IPT could be one of the techniques to assess the similarities and differences, and hence help to bridge the gap. The achievement hereof would also contribute to the development of a mechanical model for the cell that would be in agreement with several different types of cell mechanical measurements. 


\subsubsection{IPT for potential applications in nanomedicine}

In this thesis, only tumor cell lines isolated from pancreas and breast tissue have been studied. The utility of IPT for characterization of the metastatic potential should be extended to different tumor cells lines, to assess the broadness of the applicability of IPT in this respect. Also comparison with isolated cells from normal tissue or even direct analysis of cells in their tissue can be considered as next steps. With these steps it might become possible to get a better understanding of the different tumor development mechanisms.

Different drugs for cancer therapy can be pre-analyzed in cell culture to evaluate the effectiveness to control metastasis. Since only small amounts of sample are needed with IPT, many different treatments could be explored based on only one biopsy.

Besides cancer, also various other diseases involve changes in the cytoskeleton and hence could be studied with IPT. An important example is that of endothelial cells, which are involved in cardiovascular diseases. Besides studying disease affected endothelial cells, also the conditions leading to pathological states could be studied. For example, fluid shear stress is known to be an important factor. Exposing endothelial cells to controlled shear stress in a laboratory environment (e.g. a fluid channel in a microfluidic chip) and studying their response with (amongst others) IPT could contribute to new insights in the development of cardiovascular diseases.

\subsubsection{Miniaturization and automatization}

Considering the ongoing developments in Lab-on-a-chip technology, IPT could be certainly integrated in applcations involving the seeding of single cells, and their diagnosis on a cell chip. And with automation of the image acquisition and analysis, IPT could potentially even be developed into a fast drug-screening technique. 


\section{Samenvatting}

Het doel van het in dit proefschrift beschreven onderzoek was het verkrijgen van nieuw inzicht in 1) de microscopische mechanische omgeving van verschillende intracellulaire deeltjes, 2) de krachten welke de verschillende deeltjes in levende cellen drijven, 3) de heterogeniteit van het cytoskelet zoals die door ruimtelijke en tijds variaties in de deeltjes dynamica wordt gereflecteerd, en 4) de potentie van het volgen van intracellulaire deeltjes (Intracellular Particle Tracking) voor biomedische toepassingen. Deze benadering werd enerzijds verondersteld om bij te dragen tot de zoektocht naar ideale tracers: deeltjes die wegens hun oppervlakte chemie specifieke informatie kunnen openbaren, zoals de dynamica van cytoskelet componenten zoals actine en microtubule netwerken, of het reologisch gedrag van het cytoskelet. Naast deze fundamentele aspecten waren er ook verwachtingen vanuit een praktisch perspectief: het onderzoek zou de ontwikkeling van Intracellular Particle Tracking kunnen bevorderen als hulpmiddel voor de karakterisering van levende cellen via hun biomechanica. IPT zou dan worden gebruikt om onderscheid te maken tussen gezonde en zieke cellen, en/of de werking van klinisch relevante drugs te onderzoeken via hun invloed op het cytoskelet.

In Hoofdstuk 1 en 2, worden de doelstellingen van dit project nader omschreven nadat eerst een introductie is gegeven van het onderzoeksgebied van de celmechanica en de daarin gebruikte methoden. Een gedetailleerd overzicht wordt gegeven over de experimentele benaderingen en de nadruk wordt gelegd op IPT en atomaire kracht microscopie (Atomic Force Microscopy). Bovendien worden de materialen en de methodes gebruikt in dit proefschrift in detail besproken.

In Hoofdstuk 3, wordt de spatiotemporale ongelijksoortigheid in de dynamica van endogene lipidekorrels in levende Hmec-1 cellen in kaart gebracht, door een grote reeks individuele deeltjestrajecten te analyseren. Eén motivatie voor deze studie was dat de genoemde heterogeniteit de interpretatie van de gemeten gemiddelde kwadratische verplaatsingen (Mean Squared Displacements) van de deeltjes kan belemmeren. Vooral als metingen zijn gebaseerd op slechts een paar deeltjes per celsteekproef kan dit probleem zich voordoen. Daarnaast was de ongelijksoortigheid in intracellulaire dynamica ook een interessant thema op zichzelf. De gemeten amplitude van de MSDs toonde sterke variaties tussen de verschillende deeltjes. Nochtans werd er geen bewijsmateriaal gevonden voor correlaties tussen MSD en de specifieke locatie binnen de cel. Dit impliceert dat een gemiddelde MSD die voor een enkele cel representatief is, slechts vereist dat er voldoende deeltjes per cel zijn. Dit resultaat rechtvaardigt ook het gebruik van 
het gemiddelde MSDs in vergelijkende studies, zoals die in hoofdstukken 4 tot 6 worden besproken. Ook werden de lengte en de tijdschalen waarover de omvang MSD gecorreleerd blijft, bestudeerd. Ruimtelijke correlaties bleken afwezig te zijn tot aan interdeeltjes afstanden van 2 microns, hetgeen klein is op de schaal van de cel. Dit geeft aan dat de ongelijksoortigheid van zeer lokale aard is. Tot een tijdschaal van $150 \mathrm{~s}$, werden geen belangrijke veranderingen in grootte voor de MSD van hetzelfde deeltje gevonden.

In Hoofdstuk 4 en 5 werden twee soorten intracellulaire sondes bestudeerd: ballistisch geinjecteerde latexdeeltjes met carboxylaatgroepen aan het oppervlak (BIPs) en endogene korrels (EGs), beide in levende menselijke endotheelcellen. Voor EGs, toonde de MSD een soortgelijke signatuur als voor deeltjes in een visceuze vloeistof. Deze signatuur lijkt universeel te zijn voor EGs in zoogdiercellen. Bij $24^{\circ} \mathrm{C}$ (lager dan de fysiologische temperatuur) kon de grootte van de MSD alsmede de manier waarop de MSD afhangt van de correlatietijd, redelijk nauwkeurig beschreven worden met theorie voor semiflexible polymeren, welke thermische fluctuaties ondergaan. Veronderstellend dat actieve krachten bij $24^{\circ} \mathrm{C}$ te verwaarlozen zijn en dat EGs de beweging van polymeren van het cytoskelet volgen, werd uit de grootte van de MSD afgeleid dat de dynamica van EGs meest waarschijnlijk is verbonden aan die van de microtubules (MTs). Bij fysiologische temperatuur $\left(37^{\circ} \mathrm{C}\right)$ waren de MSD amplitudes veel groter, hetgeen aan actieve krachten werd toegeschreven (ondanks de schijnbaar diffusieve signatuur van de MSD). Deze veronderstellingen voor de micro omgeving en actieve drijvende krachten voor EGs konden worden bevestigd door de invloed van diverse chemische interventies op de MSD te onderzoeken. Hierbij bleek dat de dynamica van EGs gevoeliger was voor veranderingen in het netwerk van MT dan voor veranderingen in het actine netwerk. Tot slot bleek dat onder fysiologische omstandigheden, de dynamica van EGs mogelijk kan worden verklaard door buigingsfluctuaties van de MTs die op hun beurt worden veroorzaakt door polymerisatiekrachten van de MTs. Elk van deze bevindingen ondersteunen de notie dat EGs als niet-invasieve sondes voor de dynamica van het netwerk van MT kan worden gebruikt.

De ballistisch geïnjecteerde deeltjes (BIPs) vertoonden een geheel andere dynamica binnen dezelfde cellen. Ten eerste was de amplitude van de MSD veel kleiner dan voor EGs. Bij korte correlatietijden vertoonde de MSD curve een quasi-plateau, overeenkomstig van deeltjes is die in een (visco) elastische kooi zijn opgesloten. Bij langere correlatietijden werd een diffusieve MSD gevonden. De zelfde chemische interventies die ook in de studie van EGs werden gebruikt, hadden een totaal ander effect op de MSDs van BIPs, en lieten zien dat BIPs 
hoofdzakelijk ingebed moeten zijn in het actine netwerk. Additionele chemische interventies die gericht waren op het uitschakelen van actieve (niet-thermische) krachten, konden geen definitief uitsluitsel geven over het soort krachten waardoor BIPs gedreven worden ( bijvoorbeeld, thermisch of afhankelijk van ATP zoals bij actomyosin contractie) Samengevat, bevestigen deze bevindingen dat BIPs gebruikt kunnen worden om de dynamische veranderingen in het actin netwerk op te sporen.

Van microreologisch standpunt is de conclusie derhalve dat onder standaard fysiologische omstandigheden, en op tijdschalen van $0.1-10 \mathrm{~s}$, noch van EGs noch van onze BIPs kan worden verondersteld dat het dynamisch gedrag in overeenstemming is met de zogeheten Generalized Stokes Einstein relatie (GSER). Nochtans kan niet worden uitgesloten dat voorwaarden kunnen worden gevonden waar de GSER wel van toepassing is. Dit zou kunnen zijn bij voldoende korte correlatietijden, of na geschikte fysieke of chemische interventies waarmee ATP afhankelijke processen selectief geëlimineerd kunnen worden.

In hoofdstuk 6 werd onderzoek gedaan naar het gebruik van IPT als hulpmiddel om kankercellen te kenmerken als zijnde kwaadaardig of goedaardig, op grond van hun MSD. Zowel EGs als BIPs werden onderzocht als sondes, en de analyse werd toegepast op zowel alvleeskliercellen als borstcellen. In alle gevallen bleek dat in kwaadaardige cellen, de amplitude van de MSDs groter was dan in de goedaardige cellen. Dit illustreert de mogelijkheid om door gebruik van IPT een semi-kwantitatieve karakterisering van de metastatische potentieel te verkrijgen. Het analyseren van de zelfde cellen met AFM door meting van krachtverplaastings krommes openbaarde dat de duidelijke elastische moduli van de kwaadaardige cellen van de borsttumor beduidend lager waren dan voor de goedaardige. Nochtans voor de alvleeskliercellen konden de moduli van goedaardige en kwaadaardige cellen niet worden onderscheiden. Dit illustreert de toegevoegde waarde van IPT naast bestaande technieken. Aldus bleek dat IPT potentieel van betekenis kan zijn voor toepassingen in diagnose, prognose of farmacologie. 


\section{Acknowledgements}

After finishing my Master's degree in molecular bioengineering, I considered how to progress my career. Bionanotechnology is a fascinating area; however, my limited knowledge of physics and mathematics seemed a big obstacle to my career development. After thorough consideration, I decided to pursue my $\mathrm{PhD}$ study in biophysics at Twente university in 2005. In PCF, it had been a hard time for me to learn all kinds of physical terms and physical communications. However, without many people's kind help, it would not have been possible for me to complete my thesis. So I would like to express my deepest thanks to all who have helped and supported me during these four years of study.

Firstly, I would like to thank my supervisor Michel (Dr. Michel, H. G. Duits) and my promotor Frieder (Prof. dr. Frieder Mugele). Michel and Frieder: thank you for offering me this $\mathrm{PhD}$ position. Although sometimes we had cultural differences, you taught me a lot about scientific precision, deliberation, and how to plan, construct papers and give presentations.

Secondly, I would like to thank Siva (Dr. Siva A. Vanapalli) for the valuable discussions and help during the project. Thank you for picking up the starting point from a mass of threads. I also want to thank Dr. Dirk van den Ende for our valuable discussions on the rheological questions.

Special thanks are due to the members of my doctoral committee - prof. dr. Wim J. Briels, Prof. dr. Isvan Vermes, Prof. dr. Andrea Bausch and Prof. dr. Vinod Subramaniam - for reading my thesis and offering valuable comments and suggestions. I appreciate that MESA+ supported the cell stress project and allowed me to stay several extra months to finish some collaborative projects.

Thank you, Annelies, for helping me to cope with all manner of documents and procedures, such as for a visa, housing and graduation.

Special thanks also to our technicians, Mariska and Cock, for designing the piezo stages and connecting the AFM veeco controllers to the custom-designed AFM scanner.

Many thanks to the previous and present group members, Diana, Tamara, Renske, Niki, Violeta, Manuella, Helmut, Adrian, Fahong, Florent, Arun, Eko, Jacob, Dileep, Dieter, Gor, Burak, Sissi, Tarun, Hao, Chandra, Jung, Olga and Pablo. I 
very much enjoyed the time we all spent together, both during work and the team-outings.

In addition, special thanks are due to my collaborators outside the group. To Dr. Jürgen Schnekenburger, who generously offered me the tumor cell lines and the cultural materials, it was such a fortunate meeting and talking to you during the Nano2life conference. To Dr. Henk Jan van Manen, thank you for offering us the lipid staining kits and helping us to make confocal movies. To Andries van den Meer, thank you for offering me so many cell cultural material. Sorry for sometimes - in an emergency — using your materials without asking you in advance. It was a great pleasure to collaborate with you on the project. Thanks to Dr. Andre A. Poot, Dr. Severine le Gac, Prof.dr. Isvan Vermes, Dr. Louis van der Maas for the valuable discussions during the cell-stress project meeting. Many thanks to the technicians in the bio labs, Marloes Kamphuis, Paul ter Braak and Remy Wiertz, for their generous support.

To my Chinese and foreign friends outside the work, I would like to thank you all for encouraging me and supporting me during my difficult time and thank you for giving me so many happy times to be together with you.

Last but not least, to my parents, my brother, to Vincent, Marc, Geertje, Sonja, Remco and Ilze, thank you for giving me life, love, care, support, happiness, family and many many...

Yixuan or Jane $\odot$

September 2009 